Portland State University

PDXScholar

\title{
The Derivation of Hybridizable Discontinuous Galerkin Methods for Stokes Flow
}

\author{
Bernardo Cockburn \\ University of Minnesota - Twin Cities \\ Jay Gopalakrishnan \\ Portland State University, gjay@pdx.edu
}

Follow this and additional works at: https://pdxscholar.library.pdx.edu/mth_fac

Part of the Applied Mathematics Commons, and the Mathematics Commons Let us know how access to this document benefits you.

\footnotetext{
Citation Details

Cockburn, Bernardo and Gopalakrishnan, Jay, "The Derivation of Hybridizable Discontinuous Galerkin Methods for Stokes Flow" (2009). Mathematics and Statistics Faculty Publications and Presentations. 58. https://pdxscholar.library.pdx.edu/mth_fac/58
}

This Post-Print is brought to you for free and open access. It has been accepted for inclusion in Mathematics and Statistics Faculty Publications and Presentations by an authorized administrator of PDXScholar. Please contact us if we can make this document more accessible: pdxscholar@pdx.edu. 


\title{
THE DERIVATION OF HYBRIDIZABLE DISCONTINUOUS GALERKIN METHODS FOR STOKES FLOW
}

\author{
BERNARDO COCKBURN * AND JAYADEEP GOPALAKRISHNAN †
}

\begin{abstract}
In this paper, we introduce a new class of discontinuous Galerkin methods for the Stokes equations. The main feature of these methods is that they can be implemented in an efficient way through a hybridization procedure which reduces the globally coupled unknowns to certain approximations on the element boundaries. We present four ways of hybridizing the methods, which differ by the choice of the globally coupled unknowns. Classical methods for the Stokes equations can be thought of as limiting cases of these new methods.
\end{abstract}

Key words. Stokes equations, mixed methods, discontinuous Galerkin methods, hybridized methods, Lagrange multipliers

AMS subject classifications. 65N30, 65M60, 35L65

1. Introduction. This paper is devoted to the derivation of a new class of discontinuous Galerkin (DG) methods for the three-dimensional Stokes problem

$$
\begin{array}{rlr}
-\Delta \boldsymbol{u}+\operatorname{grad} p=\boldsymbol{f} & \text { in } \Omega, \\
\operatorname{div} \boldsymbol{u}=0 & \text { in } \Omega, \\
\boldsymbol{u}=\boldsymbol{g} & \text { on } \partial \Omega .
\end{array}
$$

As usual, we assume that $\boldsymbol{f}$ is in $L^{2}(\Omega)^{3}$, that $\boldsymbol{g} \in H^{1 / 2}(\partial \Omega)^{3}$ and that $\boldsymbol{g}$ satisfies the compatibility condition

$$
\int_{\partial \Omega} \boldsymbol{g} \cdot \boldsymbol{n}=0
$$

where $\boldsymbol{n}$ is the outward unit normal on $\partial \Omega$. We assume that $\Omega$ is a bounded simply connected domain with connected Lipschitz polyhedral boundary $\partial \Omega$.

The novelty in the class of DG methods derived here lies in the fact that they can be hybridized. Hybridized methods are primarily attractive due to the reduction in the number of globally coupled unknowns, especially in the high order case. Hybridization for conforming methods was traditionally thought of as a reformulation that moves the inter-element continuity constraints of approximations from the finite element spaces to the system of equations. Such reformulations are now well known to posses various advantages [9] (in addition to the reduction in the number of unknowns). In adapting the hybridization idea to DG methods, we face the difficulty that DG methods have no inter-element continuity constraints to begin with. Nonetheless, some DG methods realize inter-element coupling through constraints on numerical traces, which can be used to perform hybridization. This idea was exploited in the context of the Poissonlike equations in [10]. It will feature again in this paper, manifesting in a more complicated form suited to the Stokes system.

\footnotetext{
*School of Mathematics, University of Minnesota, Minneapolis, MN 55455, USA, email: cockburn@math.umn.edu. Supported in part by the National Science Foundation (Grant DMS0712955) and by the University of Minnesota Supercomputing Institute.

${ }^{\dagger}$ Department of Mathematics, University of Florida, Gainesville, FL 32611-8105, email: jayg@math.ufl.edu. Supported in part by the National Science Foundation under grants DMS0713833 and SCREMS-0619080.
} 
Let us put this contribution in perspective. This paper can be considered to be part of a series of papers in which we study hybridization of finite element methods. The hybridization of classical mixed methods for second-order elliptic problems was considered in $[5,6]$. Hybridization of a discontinuous Galerkin method for the two dimensional Stokes system was carried out in [3], while hybridization of a mixed method for the three dimensional Stokes system was developed in $[7,8]$. A short review of the work done up to 2005 is provided in [9].

Recently in [10] it was shown how mixed, discontinuous, continuous and even nonconforming Galerkin methods can be hybridized in a single, unifying framework. This was done for second-order elliptic problems. In this paper, we extend this approach to Galerkin methods for the Stokes problem. However, although the hybridization techniques we propose here provide a similar unifying framework, we prefer to sacrifice generality for the sake of clarity, and concentrate our efforts on a particular, new class of methods we call the hybridizable discontinuous Galerkin (HDG) methods. Then, just as was done for second-order elliptic problems in [10], we show that this procedure also applies to mixed and other classic methods which can be obtained as particular or limiting cases of these HDG methods.

Our results are also an extension of previous work on hybridization of a DG [3] and a classical mixed method $[7,8]$ for the Stokes equations. For these two methods, hybridization was used to circumvent the difficult task of constructing a local basis for divergence-free spaces for velocity. Moreover, in [7, 8], it was shown that hybridization results in a new formulation of the method which only involves the tangential velocity and the pressure on the faces of the elements. In this paper, we show that such a formulation can also be obtained for the HDG methods. We also show that these methods can be hybridized in three additional ways differing in the choice of variables which are globally coupled.

The organization of the paper is as follows. In Section 2, we present the HDG methods and show that their approximate solution is well defined. In Section 3, we present the four hybridizations of the HDG methods in full detail. Proofs of the theorems therein are displayed in Section 4. Finally, in Section 5, we end with some concluding remarks.

\section{The HDG methods.}

2.1. Definition of the methods. Let us describe the HDG methods under consideration. We begin by introducing our notation. We denote by $\Omega_{h}=\{K\}$ a subdivision of the domain $\Omega$ into shape-regular tetrahedra $K$ satisfying the usual assumptions of finite element meshes and set $\partial \Omega_{h}:=\left\{\partial K: K \in \Omega_{h}\right\}$. We associate to this mesh the set of interior faces $\mathscr{E}_{h} \mathrm{O}$ and the set of boundary faces $\mathscr{E}_{h} \partial$. We say that $e \in \mathscr{E}_{h}^{o}$ if there are two tetrahedra $K^{+}$and $K^{-}$in $\Omega_{h}$ such that $e=\partial K^{+} \cap \partial K^{-}$, and we say that $e \in \mathscr{E}_{h} \partial$ if there is a tetrahedra $K$ in $\Omega_{h}$ such that $e=\partial K \cap \partial \Omega$. We set $\mathscr{E}_{h}:=\mathscr{E}_{h} \cup \mathscr{E}_{h} \partial$

The HDG methods provide an approximate solution $\left(\boldsymbol{\omega}_{h}, \boldsymbol{u}_{h}, p_{h}\right)$ in some finite dimensional space $\boldsymbol{W}_{h} \times \boldsymbol{V}_{h} \times P_{h}$ of the form

$$
\begin{aligned}
\boldsymbol{W}_{h}=\left\{\boldsymbol{\tau} \in \boldsymbol{L}^{2}(\Omega):\right. & \left.\left.\boldsymbol{\tau}\right|_{K} \in \boldsymbol{W}(K) \quad \forall K \in \Omega_{h}\right\}, \\
\boldsymbol{V}_{h}=\left\{\boldsymbol{v} \in \boldsymbol{L}^{2}(\Omega):\right. & \left.\left.\boldsymbol{v}\right|_{K} \in \boldsymbol{V}(K) \quad \forall K \in \Omega_{h}\right\}, \\
P_{h}=\left\{q \in L^{2}(\Omega):\right. & \left.\left.q\right|_{K} \in P(K) \quad \forall K \in \Omega_{h}\right\},
\end{aligned}
$$

where the local spaces $\boldsymbol{W}(K), \boldsymbol{V}(K)$ and $P(K)$ are finite dimensional polynomial spaces that we shall specify later. 
To define the approximate solution, we use the following formulation of the Stokes equations:

$$
\begin{aligned}
\boldsymbol{\omega}-\operatorname{curl} \boldsymbol{u} & =0 & & \text { in } \Omega, \\
\operatorname{curl} \boldsymbol{\omega}+\operatorname{grad} p & =\boldsymbol{f} & & \text { in } \Omega, \\
\operatorname{div} \boldsymbol{u} & =0 & & \text { in } \Omega, \\
\boldsymbol{u} & =\boldsymbol{g} & & \text { on } \partial \Omega .
\end{aligned}
$$

Multiplying the first three equations by test functions and integrating by parts, we arrive at the following formulation for determining an approximate solution $\left(\boldsymbol{\omega}_{h}, \boldsymbol{u}_{h}, p_{h}\right)$ in $\boldsymbol{W}_{h} \times \boldsymbol{V}_{h} \times P_{h}$ :

$$
\begin{aligned}
& \left(\boldsymbol{\omega}_{h}, \boldsymbol{\tau}\right)_{\Omega_{h}}-\left(\boldsymbol{u}_{h}, \operatorname{curl} \boldsymbol{\tau}\right)_{\Omega_{h}}+\left\langle\widehat{\boldsymbol{u}}_{h}, \boldsymbol{n} \times \boldsymbol{\tau}\right\rangle_{\partial \Omega_{h}}=0, \\
& \left(\boldsymbol{\omega}_{h}, \operatorname{curl} \boldsymbol{v}\right)_{\Omega_{h}}+\left\langle\widehat{\boldsymbol{\omega}}_{h}, \boldsymbol{v} \times \boldsymbol{n}\right\rangle_{\partial \Omega_{h}} \\
& -\left(p_{h}, \operatorname{div} \boldsymbol{v}\right)_{\Omega_{h}}+\left\langle\widehat{p}_{h}, \boldsymbol{v} \cdot \boldsymbol{n}\right\rangle_{\partial \Omega_{h}}=(\boldsymbol{f}, \boldsymbol{v})_{\Omega_{h}}, \\
& -\left(\boldsymbol{u}_{h}, \operatorname{grad} q\right)_{\Omega_{h}}+\left\langle\widehat{\boldsymbol{u}}_{h} \cdot \boldsymbol{n}, q\right\rangle_{\partial \Omega_{h}}=0,
\end{aligned}
$$

for all $(\boldsymbol{\tau}, \boldsymbol{v}, q) \in \boldsymbol{W}_{h} \times \boldsymbol{V}_{h} \times P_{h}$. The notation for volume innerproducts above is defined by

$$
(\zeta, \omega)_{\Omega_{h}}:=\sum_{K \in \Omega_{h}} \int_{K} \zeta(x) \omega(x) d x \quad \text { and } \quad(\boldsymbol{\sigma}, \boldsymbol{v})_{\Omega_{h}}:=\sum_{i=1}^{3}\left(\sigma_{i}, v_{i}\right)_{\Omega_{h}}
$$

for all $\zeta, \omega$ in $L^{2}\left(\Omega_{h}\right):=\left\{v:\left.v\right|_{K} \in L^{2}(K)\right.$ for all $K$ in $\left.\Omega_{h}\right\}$, and all $\boldsymbol{\sigma}, \boldsymbol{v} \in \boldsymbol{L}^{2}\left(\Omega_{h}\right):=$ $\left[L^{2}\left(\Omega_{h}\right)\right]^{3}$. More generally, our notation is such that if $S$ represents the notation for any given space (e.g., $S$ can be $L^{2}, H^{1}$, etc.), the bold face notation $\boldsymbol{S}\left(\Omega_{h}\right)$ denotes $\left[S\left(\Omega_{h}\right)\right]^{3}$, and

$$
\begin{aligned}
S\left(\Omega_{h}\right) & :=\left\{\omega: \Omega_{h} \mapsto \mathbb{R},\left.\omega\right|_{K} \in S(K) \forall K \in \Omega_{h}\right\}, \\
S\left(\partial \Omega_{h}\right) & :=\left\{\omega: \partial \Omega_{h} \mapsto \mathbb{R},\left.\omega\right|_{\partial K} \in S(\partial K) \forall K \in \Omega_{h}\right\} .
\end{aligned}
$$

The boundary innerproducts in (2.2) are defined by

$$
\langle\boldsymbol{v} \odot \boldsymbol{n}, \mu\rangle_{\partial \Omega_{h}}:=\sum_{K \in \Omega_{h}} \int_{\partial K} \boldsymbol{v}(\gamma) \odot \boldsymbol{n} \mu(\gamma) d \gamma
$$

where $\odot$ is either $\cdot($ the dot product) or $\times$ (the cross product) and $\boldsymbol{n}$ denotes the unit outward normal vector on $\partial K$. Similarly, for any $\mathscr{F}_{h} \subseteq \mathscr{E}_{h}$, the notation $\langle\cdot, \cdot\rangle_{\mathscr{F}_{h}}$ indicates a sum of integrals over the faces in $\mathscr{F}_{h}$.

To complete the definition of the HDG methods, we need to specify the numerical traces, for which we need the following notation. For any vector-valued function $\boldsymbol{v}$ we set

$$
\begin{aligned}
\boldsymbol{v}_{t} & :=\boldsymbol{n} \times(\boldsymbol{v} \times \boldsymbol{n}), \\
\boldsymbol{v}_{n} & :=\boldsymbol{n}(\boldsymbol{v} \cdot \boldsymbol{n}) .
\end{aligned}
$$

Note that we have that $\boldsymbol{v}=\boldsymbol{v}_{n}+\boldsymbol{v}_{t}$. In this paper we will often use double valued functions on $\mathscr{E}_{h}^{o}$. One example is $\boldsymbol{n}$. Indeed, on each interior mesh face $e=\partial K^{+} \cap$ $\partial K^{-}$, the unit normal $\boldsymbol{n}$ is double valued with two branches, one from $K^{+}$, which 
we denote by $\boldsymbol{n}^{+}$, and another from $K^{-}$which we denote by $\boldsymbol{n}^{-}$. Similarly, if $\boldsymbol{v}$ is in $\boldsymbol{H}^{1}\left(\Omega_{h}\right)$, its full trace, as well as the tangential and normal traces in (2.3), are generally double valued on $\mathscr{E}_{h}$. We use $\boldsymbol{v}^{+}$and $\boldsymbol{v}^{-}$to denote the full trace on $e$ of $\boldsymbol{v}$ from $K^{+}$and $K^{-}$, respectively. On each $e=\partial K^{+} \cap \partial K^{-}$, the jumps of double valued functions $\boldsymbol{v}$ in $\boldsymbol{H}^{1}\left(\Omega_{h}\right)$ and $q$ in $H^{1}\left(\Omega_{h}\right)$ are defined by

$$
\begin{aligned}
\llbracket q \boldsymbol{n} \rrbracket & :=q^{+} \boldsymbol{n}^{+}+q^{-} \boldsymbol{n}^{-}, \\
\llbracket \boldsymbol{v} \odot \boldsymbol{n} \rrbracket & :=\boldsymbol{v}^{+} \odot \boldsymbol{n}^{+}+\boldsymbol{v}^{-} \odot \boldsymbol{n}^{-},
\end{aligned}
$$

where $\odot$ is either $\cdot$ or $\times$.

With these preparations we can now specify our definition of the numerical traces appearing in (2.2). On the interior faces $\mathscr{E}_{h}$, we set

$$
\begin{aligned}
\left(\widehat{\boldsymbol{\omega}}_{h}\right)_{t} & =\left(\frac{\tau_{t}^{-}\left(\boldsymbol{\omega}_{h}^{+}\right)_{t}+\tau_{t}^{+}\left(\boldsymbol{\omega}_{h}^{-}\right)_{t}}{\tau_{t}^{-}+\tau_{t}^{+}}\right)+\left(\frac{\tau_{t}^{+} \tau_{t}^{-}}{\tau_{t}^{-}+\tau_{t}^{+}}\right) \llbracket \boldsymbol{u}_{h} \times \boldsymbol{n} \rrbracket, \\
\left(\widehat{\boldsymbol{u}}_{h}\right)_{t} & =\left(\frac{\tau_{t}^{+}\left(\boldsymbol{u}_{h}^{+}\right)_{t}+\tau_{t}^{-}\left(\boldsymbol{u}_{h}^{-}\right)_{t}}{\tau_{t}^{-}+\tau_{t}^{+}}\right)+\left(\frac{1}{\tau_{t}^{-}+\tau_{t}^{+}}\right) \llbracket \boldsymbol{n} \times \boldsymbol{\omega}_{h} \rrbracket, \\
\left(\widehat{\boldsymbol{u}}_{h}\right)_{n} & =\left(\frac{\tau_{n}^{+}\left(\boldsymbol{u}_{h}^{+}\right)_{n}+\tau_{n}^{-}\left(\boldsymbol{u}_{h}^{-}\right)_{n}}{\tau_{n}^{-}+\tau_{n}^{+}}\right)+\left(\frac{1}{\tau_{n}^{-}+\tau_{n}^{+}}\right) \llbracket p_{h} \boldsymbol{n} \rrbracket, \\
\widehat{p}_{h} & =\left(\frac{\tau_{n}^{-} p_{h}^{+}+\tau_{n}^{+} p_{h}^{-}}{\tau_{n}^{-}+\tau_{n}^{+}}\right)+\left(\frac{\tau_{n}^{+} \tau_{n}^{-}}{\tau_{n}^{-}+\tau_{n}^{+}}\right) \llbracket \boldsymbol{u}_{h} \cdot \boldsymbol{n} \rrbracket,
\end{aligned}
$$

where the so-called penalization or stabilization parameters $\tau_{t}$ and $\tau_{n}$ are functions on $\mathscr{E}_{h}$ that are constant on each $e$ in $\mathscr{E}_{h}$ and double valued on $\mathscr{E}_{h}$; indeed, if $e=$ $\partial K^{+} \cap \partial K^{-}$, then $\tau_{t}^{ \pm}$and $\tau_{n}^{ \pm}$are the values on $e \cap \partial K^{ \pm}$of the stabilization parameters. Finally, on the boundary faces of $\mathscr{E}_{h}$, we set

$$
\begin{aligned}
\left(\widehat{\boldsymbol{u}}_{h}\right)_{t} & =\boldsymbol{g}_{t}, \\
\left(\widehat{\boldsymbol{u}}_{h}\right)_{n} & =\boldsymbol{g}_{n}, \\
\left(\widehat{\boldsymbol{\omega}}_{h}\right)_{t} & =\left(\boldsymbol{\omega}_{h}\right)_{t}+\tau_{t}\left(\boldsymbol{u}_{h}-\widehat{\boldsymbol{u}}_{h}\right) \times \boldsymbol{n}, \\
\widehat{p}_{h} & =p_{h}+\tau_{n}\left(\boldsymbol{u}_{h}-\widehat{\boldsymbol{u}}_{h}\right) \cdot \boldsymbol{n} .
\end{aligned}
$$

This completes the definition of the HDG method in (2.2), save the specification of the spaces on each element.

Let us briefly motivate the choice of the above numerical traces. First, we want them to be linear combinations of the traces of the approximate solution $\left(\boldsymbol{\omega}_{h}, \boldsymbol{u}_{h}, p_{h}\right)$. We also want them to be consistent and conservative; these are very important properties of the numerical traces as was shown in [1] in the context of second-order elliptic problems. They are consistent because when the approximate solution is continuous across interelement boundaries, or at the boundary of $\Omega$, we have that

$$
\left(\left(\widehat{\boldsymbol{\omega}}_{h}\right)_{t},\left(\widehat{\boldsymbol{u}}_{h}\right)_{t},\left(\widehat{\boldsymbol{u}}_{h}\right)_{n}, \widehat{p}_{h}\right)=\left(\left(\boldsymbol{\omega}_{h}\right)_{t},\left(\boldsymbol{u}_{h}\right)_{t},\left(\boldsymbol{u}_{h}\right)_{n}, p_{h}\right) .
$$

They are conservative because they are single valued.

The above general considerations, however, are not enough to justify the specific expression of the numerical traces on the parameters $\tau_{t}$ and $\tau_{n}$. We take this particular expression because it allows the hybridization of the methods. Although this will become evident when we develop each of its four hybridizations, we can briefly argue why this is so. Suppose that we want the numerical trace of the velocity, $\widehat{\boldsymbol{u}}_{h}=$ 
$\left(\widehat{\boldsymbol{u}}_{h}\right)_{t}+\left(\widehat{\boldsymbol{u}}_{h}\right)_{n}$, to be the globally coupled unknown. This means that, on each element $K \in \Omega_{h}$, we should be able to express all the remaining unknowns in terms of $\widehat{\boldsymbol{u}}_{h}$. If in the weak formulation defining the method, (2.2), we take test functions with support in the element $K$, we see that we can achieve this if we could write

$$
\left(\widehat{\boldsymbol{\omega}}_{h}\right)_{t}=\left(\boldsymbol{\omega}_{h}\right)_{t}+\tau_{t}\left(\boldsymbol{u}_{h}-\widehat{\boldsymbol{u}}_{h}\right) \times \boldsymbol{n} \quad \text { and } \quad \widehat{p}_{h}=p_{h}+\tau_{n}\left(\boldsymbol{u}_{h}-\widehat{\boldsymbol{u}}_{h}\right) \cdot \boldsymbol{n},
$$

where $\left(\boldsymbol{\omega}_{h}, \boldsymbol{u}_{h}, p_{h}\right)$ is the approximation on the element $K, \boldsymbol{n}$ is the outward unit normal to $K$ and $\tau_{t}$ and $\tau_{n}$ take the values associated with $K$. Note that this is consistent with the choice of the corresponding numerical traces on the border of $\Omega$, equations (2.6c) and (2.6d). Since the element $K$ was arbitrary, we should then have

$$
\begin{aligned}
\left(\widehat{\boldsymbol{\omega}}_{h}\right)_{t} & =\left(\boldsymbol{\omega}_{h}\right)_{t}^{+}+\tau_{t}^{+}\left(\boldsymbol{u}_{h}^{+}-\widehat{\boldsymbol{u}}_{h}\right) \times \boldsymbol{n}^{+}=\left(\boldsymbol{\omega}_{h}\right)_{t}^{-}+\tau_{t}^{-}\left(\boldsymbol{u}_{h}^{-}-\widehat{\boldsymbol{u}}_{h}\right) \times \boldsymbol{n}^{-}, \\
\widehat{p}_{h} & =p_{h}^{+}+\tau_{n}^{+}\left(\boldsymbol{u}_{h}^{+}-\widehat{\boldsymbol{u}}_{h}\right) \cdot \boldsymbol{n}^{+}=p_{h}^{-}+\tau_{n}^{-}\left(\boldsymbol{u}_{h}^{-}-\widehat{\boldsymbol{u}}_{h}\right) \cdot \boldsymbol{n}^{-},
\end{aligned}
$$

on all interior faces. A simple algebraic manipulation shows that this is possible only if the numerical traces therein are taken as in (2.5).

Let us end this subsection by remarking that the choice of the penalization parameters $\tau_{t}$ and $\tau_{n}$ can be crucial since it can have an important effect on both the stability and the accuracy of the method. This constitutes ongoing work; see the last paragraph of last section. In Subsection 3.5, we show how by taking special choices of these parameters, several already known methods for the Stokes system are recovered.

2.2. Other boundary conditions. The vorticity-velocity variational formulation admits imposition of boundary conditions other than (2.1d); see a short discussion in Subsection 4.3 in [16]. In this paper, we consider the following types of boundary conditions:

$$
\left.\begin{array}{rl}
\boldsymbol{u}_{t} & =\boldsymbol{g}_{t} \\
p & =r
\end{array}\right\} \quad \text { Type I boundary condition, }
$$

We have already defined the HDG method in the case of the Type II boundary condition in the previous subsection. Neither the equations of the HDG method (2.2), nor the equations of the interior numerical traces $(2.5 \mathrm{a})-(2.5 \mathrm{~d})$ change when the other boundary conditions are considered. But the equations for the boundary numerical 
traces, namely (2.6a)-(2.6d), must be changed as follows:

$$
\left.\begin{array}{rl}
\left(\widehat{\boldsymbol{u}}_{h}\right)_{t} & =\boldsymbol{g}_{t}, \\
\left(\widehat{\boldsymbol{u}}_{h}\right)_{n} & =\left(\boldsymbol{u}_{h}\right)_{n}+\frac{1}{\tau_{n}}\left(p_{h}-\widehat{p}_{h}\right) \boldsymbol{n}, \\
\widehat{\boldsymbol{\omega}}_{h} & =\left(\boldsymbol{\omega}_{h}\right)_{t}+\tau_{t}\left(\boldsymbol{u}_{h}-\widehat{\boldsymbol{u}}_{h}\right) \times \boldsymbol{n}, \\
\widehat{p}_{h} & =r \\
\left(\widehat{\boldsymbol{u}}_{h}\right)_{t} & =\left(\boldsymbol{u}_{h}\right)_{t}+\frac{1}{\tau_{t}} \boldsymbol{n} \times\left(\boldsymbol{\omega}_{h}-\widehat{\boldsymbol{\omega}}_{h}\right), \\
\left(\widehat{\boldsymbol{u}}_{h}\right)_{n} & =\boldsymbol{g}_{n}, \\
\left(\widehat{\boldsymbol{\omega}}_{h}\right)_{t} & =\gamma_{t}, \\
\widehat{p}_{h}=p_{h}+\tau_{n}\left(\boldsymbol{u}_{h}-\widehat{\boldsymbol{u}}_{h}\right) \cdot \boldsymbol{n}, & \text { for Type I, } \\
\left(\widehat{\boldsymbol{u}}_{h}\right)_{t} & =\left(\boldsymbol{u}_{h}\right)_{t}+\frac{1}{\tau_{t}} \boldsymbol{n} \times\left(\boldsymbol{\omega}_{h}-\widehat{\boldsymbol{\omega}}_{h}\right), \\
\left(\widehat{\boldsymbol{u}}_{h}\right)_{n} & =\left(\boldsymbol{u}_{h}\right)_{n}+\frac{1}{\tau_{n}}\left(p_{h}-\widehat{p}_{h}\right) \boldsymbol{n}, \\
\left(\widehat{\boldsymbol{\omega}}_{h}\right)_{t} & =\gamma_{t}, \\
\widehat{p}_{h} & =r
\end{array}\right\} \quad \text { for Type III, }
$$

When we do not have boundary conditions on pressure, the pressure variable in Stokes flow is only determined up to a constant. Therefore, for Type II and Type III boundary conditions, in order to obtain unique solvability we must change the pressure space from $P_{h}$ to

$$
P_{h}^{0}=P_{h} \cap L_{0}^{2}(\Omega),
$$

where $L_{0}^{2}(\Omega)$ is the set of functions in $L^{2}(\Omega)$ whose mean on $\Omega$ is zero. In the case of Type I and Type IV boundary conditions, the pressure space is simply $P_{h}$. Finally, let us point out that the Type IV boundary conditions are not particularly useful since they have to be complemented by additional conditions on the velocity. For this reason, we do not consider them as possible boundary conditions for the Stokes equations. However, we discuss it here because, as we are going to see, there is a oneto-one correspondence between the four types of boundary conditions just considered and the four hybridizations of the HDG method.

2.3. Existence and uniqueness of the HDG solution. With (strictly) positive penalty parameters, the HDG method is well defined, as we next show. When we say that a multi-valued function $\tau$ is positive on $\partial \Omega_{h}$, we mean that both branches of $\tau$ are positive on all faces of $\mathscr{E}_{h}^{o}$ and furthermore that the branch from within $\Omega$ is positive on the faces of $\partial \Omega$. Of course, the branch from outside $\Omega$ is zero.

To simplify our notation, we will use a symbol for averages of double valued functions. On any interior face $e=\partial K^{+} \cap \partial K^{-}$, let

$$
\left\{\{v\}_{\alpha}=v^{+} \alpha^{+}+v^{-} \alpha^{-},\right.
$$

for any double valued function $\alpha$. The notation $\{\{v\}$ (without a subscript) denotes $\left\{\{v\}_{\alpha}\right.$ with $\alpha^{+}=\alpha^{-}=1 / 2$. As a final note on our notation, we do not distinguish between functions and their extensions by zero. Accordingly, we use the previously defined notations like $\llbracket \cdot \rrbracket$ and $\left\{\{\cdot\}\right.$ even for boundary faces in $\mathscr{E}_{h}^{\partial}$ with the understanding that one of the branches involved is zero (which is the case when the function is 
extended by zero). E.g., on a boundary face $e$, the penalty function $\tau_{n}$ has only one nonzero branch, say $\tau_{n}^{-}$, so $\left\{\left\{\tau_{n}\right\}\right.$ on $e$ equals $\tau_{n}^{-} / 2$. With this notation it is easy to verify that the identities

$$
\begin{aligned}
\langle\boldsymbol{\sigma}, \boldsymbol{v} \times \boldsymbol{n}\rangle_{\partial \Omega_{h}} & =\left\langle\left\{\{\boldsymbol{\sigma}\}_{\alpha}, \llbracket \boldsymbol{v} \times \boldsymbol{n} \rrbracket\right\rangle_{\mathscr{E}_{h}}-\left\langle\llbracket \boldsymbol{\sigma} \times \boldsymbol{n} \rrbracket,\left\{\{\boldsymbol{v}\}_{1-\alpha}\right\rangle_{\mathscr{E}_{h}},\right.\right. \\
\langle q, \boldsymbol{v} \cdot \boldsymbol{n}\rangle_{\partial \Omega_{h}} & =\left\langle\{\{q\}\}_{\alpha}, \llbracket \boldsymbol{v} \cdot \boldsymbol{n} \rrbracket\right\rangle_{\mathscr{E}_{h}}+\left\langle\llbracket q \boldsymbol{n} \rrbracket,\left\{\{\boldsymbol{v}\}_{1-\alpha}\right\rangle_{\mathscr{E}_{h}},\right.
\end{aligned}
$$

hold for any $\alpha$ whose branches sum to one, i.e., $\alpha^{+}+\alpha^{-}=1$ on every face $e$ in $\mathscr{E}_{h}$.

Proposition 2.1. Assume that $\tau_{t}$ and $\tau_{n}$ are positive on $\partial \Omega_{h}$. Assume also that

$$
\begin{aligned}
\operatorname{curl} \boldsymbol{V}(K) & \subset \boldsymbol{W}(K), \\
\operatorname{grad} P(K) & \subset \boldsymbol{V}(K), \\
\operatorname{div} \boldsymbol{V}(K) & \subset P(K),
\end{aligned}
$$

for every element $K \in \Omega_{h}$. Then we have that

1. For Type I boundary condition, there is one and only one $\left(\boldsymbol{\omega}_{h}, \boldsymbol{u}_{h}, p_{h}\right)$ in the space $\boldsymbol{W}_{h} \times \boldsymbol{V}_{h} \times P_{h}$ satisfying (2.2), (2.5), and (2.8a).

2. For Type II boundary condition, there is a solution $\left(\boldsymbol{\omega}_{h}, \boldsymbol{u}_{h}, p_{h}\right)$ in the space $\boldsymbol{W}_{h} \times \boldsymbol{V}_{h} \times P_{h}$ satisfying (2.2), (2.5), and (2.6), if and only if $\boldsymbol{g}$ satisfies (1.1). When a solution $\left(\boldsymbol{\omega}_{h}, \boldsymbol{u}_{h}, p_{h}\right)$ exists, all solutions are of the form $\left(\boldsymbol{\omega}_{h}, \boldsymbol{u}_{h}, p_{h}+\kappa\right)$ for some constant function $\kappa$. There is a unique solution if $P_{h}$ is replaced by $P_{h}^{0}$.

3. For Type III, the statements of Type II case hold verbatim after replacing (2.6) with $(2.8 \mathrm{~b})$.

Proof. The proof proceeds by setting all data to zero and finding the null space in each of the three cases. Taking $(\boldsymbol{\tau}, \boldsymbol{v}, q):=\left(\boldsymbol{\omega}_{h}, \boldsymbol{u}_{h}, p_{h}\right)$ in the equations (2.2) and adding them, we obtain

$$
\left(\boldsymbol{\omega}_{h}, \boldsymbol{\omega}_{h}\right)_{\Omega_{h}}+\Theta_{h}=0
$$

where

$$
\begin{aligned}
\Theta_{h}:= & \left\langle-\boldsymbol{u}_{h}, \boldsymbol{n} \times \boldsymbol{\omega}_{h}\right\rangle_{\partial \Omega_{h}}+\left\langle\widehat{\boldsymbol{u}}_{h}, \boldsymbol{n} \times \boldsymbol{\omega}_{h}\right\rangle_{\partial \Omega_{h}}-\left\langle\boldsymbol{u}_{h}, \boldsymbol{n} \times \widehat{\boldsymbol{\omega}}_{h}\right\rangle_{\partial \Omega_{h}} \\
& -\left\langle p_{h}, \boldsymbol{u}_{h} \cdot \boldsymbol{n}\right\rangle_{\partial \Omega_{h}}+\left\langle\widehat{p}_{h}, \boldsymbol{u}_{h} \cdot \boldsymbol{n}\right\rangle_{\partial \Omega_{h}}+\left\langle p_{h}, \widehat{\boldsymbol{u}}_{h} \cdot \boldsymbol{n}\right\rangle_{\partial \Omega_{h}} .
\end{aligned}
$$

Rewriting $\Theta_{h}$ using (2.9), we obtain

$$
\begin{aligned}
\Theta_{h}= & -\left\langle\widehat{\boldsymbol{\omega}}_{h}-\left\{\left\{\boldsymbol{\omega}_{h}\right\}_{1-\alpha}, \llbracket \boldsymbol{n} \times \boldsymbol{u}_{h} \rrbracket\right\rangle_{\mathscr{E}_{h}}+\left\langle\widehat{\boldsymbol{u}}_{h}-\left\{\left\{\boldsymbol{u}_{h}\right\}_{\alpha}, \llbracket \boldsymbol{n} \times \widehat{\boldsymbol{\omega}}_{h} \rrbracket\right\rangle_{\mathscr{E}_{h}}\right.\right. \\
& +\left\langle\widehat{p}_{h}-\left\{\left\{p_{h}\right\}_{1-\beta}, \llbracket \boldsymbol{u} \boldsymbol{u}_{h} \cdot \boldsymbol{n} \rrbracket\right\rangle_{\mathscr{E}_{h}}+\left\langle\widehat{\boldsymbol{u}}_{h}-\left\{\left\{\boldsymbol{u}_{h}\right\}_{\beta}, \llbracket p_{h} \boldsymbol{n} \rrbracket\right\rangle_{\mathscr{E}_{h}},\right.\right.
\end{aligned}
$$

for any $\alpha$ and $\beta$ whose branches sum to one on every face of $\mathscr{E}_{h}$. We set $\alpha=\tau_{t} / 2\left\{\left\{\tau_{t}\right\}\right\}$ and $\beta=\tau_{n} / 2\left\{\left\{\tau_{n}\right\}\right.$ on all the interior faces of $\mathscr{E}_{h}$. On the remaining boundary faces, we set $\alpha$ and $\beta$ case by case as follows, letting $\alpha_{\partial \Omega}^{-}, \beta_{\partial \Omega}^{-}$and $\alpha_{\partial \Omega}^{+}, \beta_{\partial \Omega}^{+}$denote the branches of $\alpha, \beta$ from outside and inside $\Omega$, respectively.

For the Type I case, we set $\alpha_{\partial \Omega}^{+}=0, \alpha_{\partial \Omega}^{-}=1, \beta_{\partial \Omega}^{+}=1, \beta_{\partial \Omega}^{-}=0$. Then, inserting the expressions for the interior and boundary numerical traces given by (2.5) and $(2.8 \mathrm{a})$,

$$
\Theta_{h}=\Theta_{h}^{o}+\left\langle\tau_{t},\left|\boldsymbol{u}_{h} \times \boldsymbol{n}\right|^{2}\right\rangle_{\partial \Omega}+\left\langle\tau_{n},\left|p_{h} \boldsymbol{n}\right|^{2}\right\rangle_{\partial \Omega}
$$


where

$$
\begin{aligned}
\Theta_{h}^{o} & =\left\langle\frac{2}{\left\{\left\{\tau_{t}\right\}\right\}},\left|\llbracket \boldsymbol{n} \times \boldsymbol{\omega}_{h} \rrbracket\right|^{2}\right\rangle_{\mathscr{E}_{h}^{o}}+\left\langle\frac{2}{\left\{\left\{1 / \tau_{t}\right\}\right.},\left|\llbracket \boldsymbol{u}_{h} \times \boldsymbol{n} \rrbracket\right|^{2}\right\rangle_{\mathscr{E}_{h}^{o}} \\
& +\left\langle\frac{2}{\left\{\left\{1 / \tau_{n}\right\}\right\}}, \llbracket \boldsymbol{u}_{h} \cdot \boldsymbol{n} \rrbracket^{2}\right\rangle_{\mathscr{E}_{h}}+\left\langle\frac{2}{\left\{\left\{\tau_{n}\right\}\right\}},\left|\llbracket p_{h} \boldsymbol{n} \rrbracket\right|^{2}\right\rangle_{\mathscr{E}_{h}} .
\end{aligned}
$$

Hence (2.10) implies that $\boldsymbol{\omega}_{h}$ vanishes, $\boldsymbol{u}_{h}$ and $p_{h}$ are continuous on $\Omega$, and $\left(\boldsymbol{u}_{h}\right)_{t}$ and $p_{h}$ vanishes on $\partial \Omega$. With this in mind, we integrate by parts the equations defining the method, namely (2.2), to obtain

$$
\begin{array}{r}
\left(\operatorname{curl} \boldsymbol{u}_{h}, \boldsymbol{\tau}\right)_{\Omega_{h}}=0, \\
\left(\operatorname{grad} p_{h}, \boldsymbol{v}\right)_{\Omega_{h}}=0, \\
\quad\left(\operatorname{div} \boldsymbol{u}_{h}, q\right)_{\Omega_{h}}=0,
\end{array}
$$

for all $(\boldsymbol{\tau}, \boldsymbol{v}, q) \in \boldsymbol{W}_{h} \times \boldsymbol{V}_{h} \times P_{h}$. By our assumptions on the local spaces, this implies that the following (global) distributional derivatives on $\Omega$ vanish:

$$
\operatorname{grad} p_{h}=\mathbf{0}, \quad \operatorname{div} \boldsymbol{u}_{h}=0 \quad \text { and } \quad \operatorname{curl} \boldsymbol{u}_{h}=\mathbf{0}
$$

The first equality implies that $p_{h}$ vanishes since we already found $p_{h}$ to vanish on $\partial \Omega$. Moreover, since $\left(\boldsymbol{u}_{h}\right)_{t}$ vanishes on the boundary $\partial \Omega$, and since we have assumed that $\partial \Omega$ consists of just one connected component, the last two equalities imply that $\boldsymbol{u}_{h}=0$. Thus, the null space is trivial.

For the Type II case, we set $\alpha_{\partial \Omega}^{+}=0, \alpha_{\partial \Omega}^{-}=1, \beta_{\partial \Omega}^{+}=0, \beta_{\partial \Omega}^{-}=1$ and simplify $\Theta_{h}$ using the interior and boundary numerical traces given by (2.5) and (2.6) to find that

$$
\Theta_{h}=\Theta_{h}^{o}+\left\langle\tau_{t},\left|\boldsymbol{u}_{h} \times \boldsymbol{n}\right|^{2}\right\rangle_{\partial \Omega}+\left\langle\tau_{n},\left|\boldsymbol{u}_{h} \cdot \boldsymbol{n}\right|^{2}\right\rangle_{\partial \Omega} .
$$

Hence (2.10) implies that $\boldsymbol{\omega}_{h}$ vanishes, $\boldsymbol{u}_{h}$ is continuous on $\Omega$, and $\boldsymbol{u}_{h}$ is zero on $\partial \Omega$, and $p_{h}$ is continuous on $\Omega$. Proceeding as in the Type I case, we find that (2.11) holds, so $\boldsymbol{u}_{h}$ vanishes. But unlike the Type I case, we can now only conclude that $p_{h}$ is constant. Thus the null space consists of $\left(\boldsymbol{\omega}_{h}, \boldsymbol{u}_{h}, p_{h}\right)=(\mathbf{0}, \mathbf{0}, \kappa)$ for constant functions $\kappa$. Hence, all statements of the proposition on the Type II case follow.

The Type III case is proved similarly.

It is interesting to note that the proof of Type II case required only minimal topological assumptions on $\Omega$, namely that $\Omega$ is connected. However, the proof of the other two cases used the further assumptions we placed on $\Omega$. The mixed method presented in [8] without such topological assumptions dealt only with Type II boundary conditions.

We can now give some possible choices for polynomial spaces that can be set within each element. Clearly, Proposition 2.1 gives the conditions that we must satisfy. Let $\mathcal{P}_{d}$ denote the space of polynomials of degree at most $d$ and let $\mathcal{P}_{d}$ denote the space of vector functions whose components are polynomials in $\mathcal{P}_{d}$. Let $d_{P} \geq 1, d_{V} \geq 0, d_{W} \geq 0$ be some integers satisfying

$$
d_{P}-1 \leq d_{V} \leq \min \left(d_{P}+1, d_{W}+1\right) .
$$

Then if we set

$$
\boldsymbol{W}(K)=\mathcal{P}_{d_{W}}, \quad \boldsymbol{V}(K)=\mathcal{P}_{d_{V}}, \quad P(K)=\mathcal{P}_{d_{P}}
$$


the conditions of Proposition 2.1 are satisfied. Some examples are

$$
\left(d_{W}, d_{V}, d_{P}\right)=\left\{\begin{array}{ccccccccc}
(k-1, & k-1, & k), & (k, & k-1, & k), & (k+1, & k-1, & k), \\
(k-1, & k, & k), & (k, & k, & k), & (k+1, & k, & k),
\end{array}\right.
$$

for some integer $k \geq 1$. Clearly there is greater flexibility in the choice of spaces than, for instance, in the mixed methods for the Stokes problem. E.g., from (2.12) it is clear that we can choose $d_{W}$ to be as large as we wish and the method continues to be well defined.

Having established that the HDG methods are well defined, we show in the next section, that they can be hybridized in different ways according to the choice of variables that are globally coupled.

3. Hybridizations of the HDG methods. In this section, we will restrict ourselves to considering the Stokes problem with the Type II boundary condition. We hybridize the HDG method for this case. As we shall see, while hybridizing we can choose to set HDG methods with the other types of boundary conditions within mesh elements.

For constructing hybridized methods based on the vorticity-velocity formulation, let us recall the following four transmission conditions for the Stokes solution components:

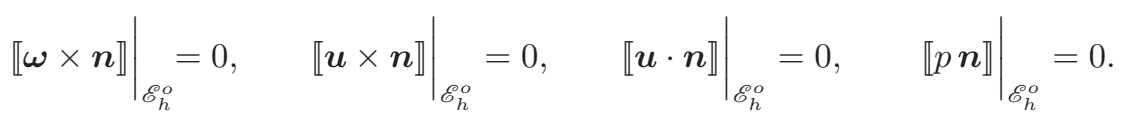

Corresponding to these four transmission conditions, there are four variables on which boundary conditions of the following form can be prescribed:

$$
\boldsymbol{\omega}_{t}=\gamma_{t}, \quad \boldsymbol{u}_{t}=\boldsymbol{\lambda}_{t}, \quad \boldsymbol{u}_{n}=\boldsymbol{\lambda}_{n}, \quad p=\rho .
$$

With this correspondence in view, we can describe our approach for constructing hybridization techniques as follows. We pick any two of the variables in (3.2) as unknown boundary values on the boundary of each mesh element. (Once these values are known, the solution inside the element can be computed locally.) Then, we formulate a global system of equations for the picked unknown variables, using the transmission conditions on the other two variables in (3.1). Of course, we must identify the proper discrete versions of these transmission conditions for this purpose. According to this strategy, there appears to be six possible cases. But two of the six cases yield underdetermined or overdetermined systems. For instance if we pick $\gamma_{t}$ and $\boldsymbol{\lambda}_{t}$ as unknowns, counting their components, we would have a total of four scalar unknown functions. However, the transmission conditions (the last two in (3.1)) form only two scalar equations, so will yield an underdetermined system. Similarly, if we pick $\boldsymbol{\lambda}_{n}$ and $\rho$ as the unknowns, we get an overdetermined system. We discard these two possibilities. In the remainder, we now work out the specifics for the remaining four cases.

3.1. Hybridization of type I. A formulation with tangential velocity and pressure. Here, we choose the second and the last of the variables in (3.2), namely $(\boldsymbol{u})_{t}$ and $p$, as the unknowns on the mesh interfaces. Their discrete approximations will be denoted by $\boldsymbol{\lambda}_{t}$ and $\rho$, respectively. We shall then use the transmission conditions 
on the other two variables, namely

$$
\llbracket \boldsymbol{\omega} \times\left.\boldsymbol{n} \rrbracket\right|_{\mathscr{E}_{h}^{\circ}}=0, \quad \text { and }\left.\quad \llbracket \boldsymbol{u} \cdot \boldsymbol{n} \rrbracket\right|_{\mathscr{E}_{h}^{\circ}}=0
$$

to derive a hybridized formulation that will help us solve for the approximations $\boldsymbol{\lambda}_{t}$ and $\rho$.

The success of this approach relies on us being able to compute approximate solutions within each element locally, once the discrete approximations $\boldsymbol{\lambda}_{t} \approx \boldsymbol{u}_{t}$ and $\rho \approx p$ are found. In other words, we need a discretization of the following Stokes problem on one element:

$$
\begin{aligned}
\boldsymbol{\omega}_{K}-\operatorname{curl} \boldsymbol{u}_{K} & =0 & & \text { in } K, \\
\operatorname{curl} \boldsymbol{\omega}_{K}+\operatorname{grad} p_{K} & =\boldsymbol{f} & & \text { in } K, \\
\operatorname{div} \boldsymbol{u}_{K} & =0 & & \text { in } K, \\
\left(\boldsymbol{u}_{K}\right)_{t} & =\boldsymbol{\lambda}_{t} & & \text { on } \partial K, \\
p_{K} & =\rho & & \text { on } \partial K .
\end{aligned}
$$

We use the HDG method (with Type I boundary conditions) applied to a single element as our discretization. Specifically, given $\left(\boldsymbol{\lambda}_{t}, \rho, \boldsymbol{f}\right)$ in $\boldsymbol{L}^{2}\left(\partial \Omega_{h}\right) \times L^{2}\left(\partial \Omega_{h}\right) \times$ $\boldsymbol{L}^{2}(\Omega)$, we define $(\mathcal{W}, \mathcal{U}, \mathcal{P})$ in $\boldsymbol{W}_{h} \times \boldsymbol{V}_{h} \times P_{h}$ on the element $K \in \Omega_{h}$ as the function in $\boldsymbol{W}(K) \times \boldsymbol{V}(K) \times P(K)$ satisfying

$$
\begin{aligned}
(\mathcal{W}, \boldsymbol{\tau})_{K}-(\mathcal{U}, \operatorname{curl} \boldsymbol{\tau})_{K} & =-\left\langle\boldsymbol{\lambda}_{t}, \boldsymbol{n} \times \boldsymbol{\tau}\right\rangle_{\partial K}, \\
(\boldsymbol{\mathcal { W }}, \operatorname{curl} \boldsymbol{v})_{K}+\langle\widehat{\mathcal{W}}, \boldsymbol{v} \times \boldsymbol{n}\rangle_{\partial K} & =(\boldsymbol{f}, \boldsymbol{v})_{K}-\langle\rho, \boldsymbol{v} \cdot \boldsymbol{n}\rangle_{\partial K}, \\
-(\mathcal{P}, \operatorname{div} \boldsymbol{v})_{K} & =0 . \\
-(\boldsymbol{U}, \operatorname{grad} q)_{K}+\langle\widehat{\mathcal{U}} \cdot \boldsymbol{n}, q\rangle_{\partial K} & =
\end{aligned}
$$

for all $(\boldsymbol{\tau}, \boldsymbol{v}, q) \in \boldsymbol{W}(K) \times \boldsymbol{V}(K) \times P(K)$, where

$$
\begin{aligned}
& (\widehat{\mathfrak{U}})_{n}=(\mathfrak{U})_{n}+\frac{1}{\tau_{n}}(\mathcal{P}-\rho) \boldsymbol{n}, \\
& \widehat{\mathcal{W}}=\mathcal{W}+\tau_{t}\left(\mathcal{U}-\boldsymbol{\lambda}_{t}\right) \times \boldsymbol{n} .
\end{aligned}
$$

Note that the above system (3.4) is obtained from the HDG system (2.2) with $\Omega$ set to $K$, and the numerical traces set by (2.8a) (and there are no interior faces). The above system of equations thus defines a linear map (the "local solver")

$$
\left(\boldsymbol{\lambda}_{t}, \rho, \boldsymbol{f}\right) \stackrel{\mathcal{L}^{\mathrm{I}}}{\longmapsto}(\mathcal{W}, \mathcal{U}, \mathcal{P})
$$

due to the unique solvability of the HDG method on one element, as given by Proposition 2.1(1).

Next, we identify conditions on $\boldsymbol{\lambda}_{t}$ and $\rho$ that make $(\mathcal{W}, \mathcal{U}, \mathcal{P})$ identical to the approximation $\left(\boldsymbol{\omega}_{h}, \boldsymbol{u}_{h}, p_{h}\right)$. We begin by restricting the function $\left(\boldsymbol{\lambda}_{t}, \rho\right)$ to the space $\left(\boldsymbol{M}_{h}\right)_{t} \times \Psi_{h}$, where

$$
\begin{aligned}
\left(\boldsymbol{M}_{h}\right)_{t} & :=\left\{\boldsymbol{\mu}_{t} \in \boldsymbol{L}^{2}\left(\mathscr{E}_{h}\right):\left.\quad \boldsymbol{\mu}_{t}\right|_{e} \in \boldsymbol{M}(e) \quad \forall e \in \mathscr{E}_{h}\right\}, \\
\Psi_{h} & :=\left\{\psi \in L^{2}\left(\mathscr{E}_{h}\right):\left.\quad \psi\right|_{e} \in \Psi(e) \quad \forall e \in \mathscr{E}_{h}\right\},
\end{aligned}
$$


where, on each face $e \in \mathscr{E}_{h}$, the finite dimensional spaces $\boldsymbol{M}(e)$ and $\Psi(e)$ are such that

$$
\begin{aligned}
\boldsymbol{M}(e) & \supseteq\left\{\left.\left(\boldsymbol{v}_{t}+\boldsymbol{n} \times \boldsymbol{\tau}\right)\right|_{e}:(\boldsymbol{\tau}, \boldsymbol{v}) \in \boldsymbol{W}(K) \times \boldsymbol{V}(K) \quad \forall K: e \subset \partial K\right\}, \\
\Psi(e) & \supseteq\left\{\left.(q+\boldsymbol{v} \cdot \boldsymbol{n})\right|_{e}:(\boldsymbol{v}, q) \in \boldsymbol{V}(K) \times P(K) \quad \forall K: e \subset \partial K\right\} .
\end{aligned}
$$

The next theorem identifies discrete analogues of the transmission conditions (3.3) as the requirements for recovering the discrete solution.

Theorem 3.1 (Conditions for type I hybridization). Suppose $\left(\boldsymbol{\omega}_{h}, \boldsymbol{u}_{h}, p_{h}\right)$ is the solution of the HDG method defined by (2.2), (2.5), and (2.6). Assume that $\left(\boldsymbol{\lambda}_{t}, \rho\right) \in\left(\boldsymbol{M}_{h}\right)_{t} \times \Psi_{h}$ is such that

$$
\begin{aligned}
\boldsymbol{\lambda}_{t} & =\boldsymbol{g}_{t} & & \text { on } \partial \Omega, \\
\left\langle\llbracket \boldsymbol{n} \times \widehat{\mathcal{W}} \rrbracket, \boldsymbol{\mu}_{t}\right\rangle_{\mathscr{E}_{h}^{o}} & =0 & & \forall \boldsymbol{\mu}_{t} \in \boldsymbol{M}_{h}, \\
\langle\llbracket \widehat{\boldsymbol{U}} \cdot \boldsymbol{n} \rrbracket, \psi\rangle_{\mathscr{E}_{h}} & =\langle\boldsymbol{g} \cdot \boldsymbol{n}, \psi\rangle_{\partial \Omega} & & \forall \psi \in \Psi_{h}, \\
(\mathcal{P}, 1)_{\Omega} & =0 . & &
\end{aligned}
$$

Then $(\mathcal{W}, \mathcal{U}, \mathcal{P})=\left(\boldsymbol{\omega}_{h}, \boldsymbol{u}_{h}, p_{h}\right), \boldsymbol{\lambda}_{t}=\left(\widehat{\boldsymbol{u}}_{h}\right)_{t}$, and $\rho=\widehat{p}_{h}$.

Proof. We begin by noting that $(\mathcal{W}, \mathcal{U}, \mathcal{P})$ is in the space $\boldsymbol{W}_{h} \times \boldsymbol{V}_{h} \times P_{h}$, by the definition of the local solvers. Moreover, by adding the equations defining the local solver, namely $(3.4 \mathrm{a})-(3.4 \mathrm{c})$, we find that $(\mathcal{W}, \mathcal{U}, \mathcal{P})$ satisfies the equations of $(2.2)$, with $(\widehat{\boldsymbol{\mathcal { W }}})_{t}$ in place of $\left(\widehat{\boldsymbol{\omega}}_{h}\right)_{t}, \boldsymbol{\lambda}_{t}$ in place of $\left(\widehat{\boldsymbol{u}}_{h}\right)_{t},(\widehat{\boldsymbol{\mathcal { U }}})_{n}$ in place of $\left(\widehat{\boldsymbol{u}}_{h}\right)_{t}$, and $\rho$ in place of $\widehat{p}_{h}$. Hence, if we show that the $(\widehat{\mathcal{W}})_{t}, \boldsymbol{\lambda}_{t},(\widehat{\mathfrak{U}})_{n}$, and $\rho$ can be related to $(\mathcal{W}, \mathcal{U}, \mathcal{P})$ as in the expressions for the numerical traces $(2.5 \mathrm{a})-(2.5 \mathrm{~d})$, then the proof will be complete because of the uniqueness result of Proposition 2.1(2) (which applies due to condition (3.6d)).

Therefore, let us first derive such expressions for $\boldsymbol{\lambda}_{t}$ and $\rho$. By the choice of the space $\boldsymbol{M}_{h} \times \Psi_{h}$, the jump conditions (3.6b) and (3.6c) imply that

$$
\llbracket \boldsymbol{n} \times \widehat{\mathcal{W}} \rrbracket=0 \quad \text { and } \quad \llbracket \widehat{\mathcal{U}} \cdot \boldsymbol{n} \rrbracket=0 \quad \text { on } \mathscr{E}_{h} .
$$

Inserting the definition of the numerical traces $(3.4 \mathrm{~d})$ and $(3.4 \mathrm{e})$, we readily obtain that, on $\mathscr{E}_{h}$,

$$
\begin{array}{r}
\llbracket \boldsymbol{n} \times \mathcal{W} \rrbracket+\tau_{t}^{+}\left(\mathfrak{U}^{+}\right)_{t}+\tau_{t}^{-}\left(\mathfrak{U}^{-}\right)_{t}-\left(\tau_{t}^{+}+\tau_{t}^{-}\right) \boldsymbol{\lambda}_{t}=0, \\
\llbracket \mathcal{U} \cdot \boldsymbol{n} \rrbracket+\frac{1}{\tau_{n}^{+}} \mathcal{P}^{+}+\frac{1}{\tau_{n}^{-}} \mathcal{P}^{-}-\left(\frac{1}{\tau_{n}^{+}}+\frac{1}{\tau_{n}^{-}}\right) \rho=0,
\end{array}
$$

or, equivalently,

$$
\begin{aligned}
\boldsymbol{\lambda}_{t} & =\left(\frac{\tau_{t}^{+}\left(\mathfrak{U}^{+}\right)_{t}+\tau_{t}^{-}\left(\mathfrak{U}^{-}\right)_{t}}{\tau_{t}^{-}+\tau_{t}^{+}}\right)+\left(\frac{1}{\tau_{t}^{-}+\tau_{t}^{+}}\right) \llbracket \boldsymbol{n} \times \mathfrak{W} \rrbracket, \\
\rho & =\left(\frac{\tau_{n}^{-} \mathcal{P}^{+}+\tau_{n}^{+} \mathcal{P}^{-}}{\tau_{n}^{-}+\tau_{n}^{+}}\right)+\left(\frac{\tau_{n}^{-} \tau_{n}^{+}}{\tau_{n}^{-}+\tau_{n}^{+}}\right) \llbracket \mathfrak{U} \cdot \boldsymbol{n} \rrbracket .
\end{aligned}
$$

Substituting these expressions into (3.4d) and (3.4e),

$$
\begin{aligned}
(\widehat{\mathcal{W}})_{t} & =\left(\frac{\tau_{t}^{-}\left(\mathcal{W}^{+}\right)_{t}+\tau_{t}^{+}\left(\mathcal{W}^{-}\right)_{t}}{\tau_{t}^{-}+\tau_{t}^{+}}\right)+\left(\frac{\tau_{t}^{+} \tau_{t}^{-}}{\tau_{t}^{-}+\tau_{t}^{+}}\right) \llbracket \mathfrak{U} \times \boldsymbol{n} \rrbracket \\
(\widehat{\mathfrak{U}})_{n} & =\left(\frac{\tau_{n}^{+}\left(\mathfrak{U}^{+}\right)_{n}+\tau_{n}^{-}\left(\mathfrak{U}^{-}\right)_{n}}{\tau_{n}^{-}+\tau_{n}^{+}}\right)+\left(\frac{1}{\tau_{n}^{-}+\tau_{n}^{+}}\right) \llbracket \mathcal{P} \boldsymbol{n} \rrbracket .
\end{aligned}
$$


In other words, the numerical traces satisfy the equations of (2.5). The fact that they satisfy the equations (2.6a) and (2.6b) follows from conditions (3.6a) and (3.6c), respectively. Finally, the equations (2.6c) and (2.6d) follow directly from the definition of the numerical traces of the local solvers $(3.4 \mathrm{e})$ and (3.4d), respectively.

Thus, by the uniqueness result of Proposition 2.1(2), we now conclude that $(\mathcal{W}, \mathcal{U}, \mathcal{P})$ coincides with $\left(\boldsymbol{\omega}_{h}, \boldsymbol{u}_{h}, p_{h}\right)$, and consequently, $\boldsymbol{\lambda}_{t}=\left(\widehat{\boldsymbol{u}}_{h}\right)_{t}$ and $\rho=\widehat{p}_{h}$. This completes the proof.

At this point, we can comment more on our strategy for construction of hybridized DG methods. Roughly speaking, the derivation of our hybridized methods proceeds by imposing discrete versions of all four transmission conditions in (3.1) through the four numerical traces of the HDG method. The two numerical traces we picked as unknowns in this case, namely $\boldsymbol{\lambda}_{t}$ and $\rho$, being single valued on $\mathscr{E}_{h}$, already satisfy a zero-jump transmission condition, so we have in some sense, already imposed the second and the fourth of the conditions in (3.1). The discrete analogues of the remaining two (the first and the third) transmission conditions are (3.6b) and (3.6c), which requires the remaining two numerical traces to be single valued. Theorem 3.1 shows that once these conditions are imposed, the HDG solution is recovered.

Next, we give a characterization of unknown traces $\boldsymbol{\lambda}_{t}$ and $\rho$ and the discrete HDG solution $\left(\boldsymbol{\omega}_{h}, \boldsymbol{u}_{h}, p_{h}\right)$ in terms of the local solvers. In particular, we show that the jump conditions (3.6b) and (3.6c) define a mixed method for the tangential velocity and the pressure. To state the result, we need to introduce some notation. Letting $\boldsymbol{\lambda}_{t}^{o}=\left.\boldsymbol{\lambda}_{t}\right|_{\mathscr{E}_{h}^{o}}$, and remembering our identification of functions with their zero extension, we can write

$$
\lambda_{t}=\lambda_{t}^{o}+g_{t}
$$

We denote by $\left(\boldsymbol{M}_{h}^{o}\right)_{t}$ the functions of $\left(\boldsymbol{M}_{h}\right)_{t}$ which are zero on $\partial \Omega$ (so $\boldsymbol{\lambda}_{t}^{o}$ is in $\left(\boldsymbol{M}_{h}^{o}\right)_{t}$ ). Finally, we use the following notation for certain specific local solutions

$$
\begin{aligned}
\left(\mathcal{W}_{\boldsymbol{\lambda}_{t}}, \mathcal{U}_{\boldsymbol{\lambda}_{t}}, \mathcal{P}_{\boldsymbol{\lambda}_{t}}\right) & :=\mathcal{L}^{\mathrm{I}}\left(\boldsymbol{\lambda}_{t}, 0, \mathbf{0}\right), \\
\left(\mathcal{W}_{\rho}, \mathcal{U}_{\rho}, \mathcal{P}_{\rho}\right) & :=\mathcal{L}^{\mathrm{I}}(\mathbf{0}, \rho, \mathbf{0}), \\
\left(\mathcal{W}_{\boldsymbol{f}}, \mathcal{U}_{\boldsymbol{f}}, \mathcal{P}_{\boldsymbol{f}}\right) & :=\mathcal{L}^{\mathrm{I}}(\mathbf{0}, 0, \boldsymbol{f}),
\end{aligned}
$$

where $\mathcal{L}^{\mathrm{I}}$ is as in (3.4f). We are now ready to state our main result for this case.

Theorem 3.2 (Characterization of the approximate solution). We have that

$$
\begin{aligned}
& \boldsymbol{\omega}_{h}=\mathcal{W}_{\boldsymbol{\lambda}_{t}^{o}}+\mathcal{W}_{\rho}+\mathcal{W}_{\boldsymbol{f}}+\mathcal{W}_{\boldsymbol{g}_{t}}, \\
& \boldsymbol{u}_{h}=\mathcal{U}_{\boldsymbol{\lambda}_{t}^{o}}+\mathcal{U}_{\rho}+\mathcal{U}_{\boldsymbol{f}}+\boldsymbol{U}_{\boldsymbol{g}_{t}}, \\
& p_{h}=\mathcal{P}_{\boldsymbol{\lambda}_{t}^{o}}+\mathcal{P}_{\rho}+\mathcal{P}_{\boldsymbol{f}}+\mathcal{P}_{\boldsymbol{g}_{t}},
\end{aligned}
$$

where $\left(\boldsymbol{\lambda}_{t}^{o}, \rho\right)$ is the only element of $\left(\boldsymbol{M}_{h}^{o}\right)_{t} \times \Psi_{h}$ such that

$$
\begin{aligned}
a_{h}\left(\boldsymbol{\lambda}_{t}^{o}, \boldsymbol{\mu}_{t}\right)+b_{h}\left(\rho, \boldsymbol{\mu}_{t}\right) & =\ell_{1}\left(\boldsymbol{\mu}_{t}\right), \\
-b_{h}\left(\psi, \boldsymbol{\lambda}_{t}^{o}\right)+c_{h}(\rho, \psi) & =\ell_{2}(\psi),
\end{aligned}
$$

for all $\left(\boldsymbol{\mu}_{t}, \psi\right) \in\left(\boldsymbol{M}_{h}^{o}\right)_{t} \times \Psi_{h}$, and

$$
\left(\mathcal{P}_{\boldsymbol{\lambda}_{t}^{o}}+\mathcal{P}_{\rho}+\mathcal{P}_{\boldsymbol{f}}+\mathcal{P}_{\boldsymbol{g}_{t}}, 1\right)_{\Omega}=0
$$


Here

$$
\begin{aligned}
a_{h}\left(\boldsymbol{\lambda}_{t}, \boldsymbol{\mu}_{t}\right) & :=\left(\mathcal{W}_{\boldsymbol{\lambda}_{t}}, \mathcal{W}_{\boldsymbol{\mu}_{t}}\right)_{\Omega_{h}}+\left\langle\tau_{t}\left(\boldsymbol{\lambda}_{t}-\mathcal{U}_{\boldsymbol{\lambda}_{t}}\right)_{t},\left(\boldsymbol{\mu}_{t}-\mathcal{U}_{\boldsymbol{\mu}_{t}}\right)_{t}\right\rangle_{\partial \Omega_{h}}+\left\langle\frac{1}{\tau_{n}} \mathcal{P}_{\boldsymbol{\lambda}_{t}}, \mathcal{P}_{\boldsymbol{\mu}_{t}}\right\rangle_{\partial \Omega_{h}} \\
b_{h}\left(\rho, \boldsymbol{\mu}_{t}\right) & :=\left\langle\rho, \boldsymbol{n} \cdot \boldsymbol{U}_{\boldsymbol{\mu}_{t}}+\frac{1}{\tau_{n}} \mathcal{P}_{\boldsymbol{\mu}_{t}}\right\rangle_{\partial \Omega_{h}}, \\
c_{h}(\rho, \psi) & :=\left(\mathcal{W}_{\rho}, \mathcal{W}_{\psi}\right)_{\Omega_{h}}+\left\langle\tau_{t}\left(\boldsymbol{U}_{\rho}\right)_{t},\left(\boldsymbol{U}_{\psi}\right)_{t}\right\rangle_{\partial \Omega_{h}}+\left\langle\frac{1}{\tau_{n}}\left(\rho-\mathcal{P}_{\rho}\right),\left(\psi-\mathcal{P}_{\psi}\right)\right\rangle_{\partial \Omega_{h}}
\end{aligned}
$$

and

$$
\begin{aligned}
\ell_{1}\left(\boldsymbol{\mu}_{t}\right) & :=\left(\boldsymbol{f}, \mathcal{U}_{\boldsymbol{\mu}_{t}}\right)_{\Omega_{h}}-a_{h}\left(\boldsymbol{g}, \boldsymbol{\mu}_{t}\right) \\
\ell_{2}(\psi) & :=-\left(\boldsymbol{f}, \mathcal{U}_{\psi}\right)_{\Omega_{h}}-\langle\boldsymbol{g} \cdot \boldsymbol{n}, \psi\rangle_{\partial \Omega}+b_{h}\left(\psi, \boldsymbol{g}_{t}\right)
\end{aligned}
$$

The proof of this theorem is in Section 4. In view of this theorem, we can obtain the HDG solution by first solving a symmetric global system that is smaller than (2.2) and then locally recovering all solution components (by applying $\mathcal{L}^{\mathbb{I}}$ ). This is the main advantage brought about by hybridization. It makes this HDG method competitive in comparison with other existing DG methods for Stokes flow.

It is interesting to note that the space in which the trace variables lie, namely $\left(\boldsymbol{M}_{h}\right)_{t}$ and $\Psi_{h}$ can be arbitrarily large. While it is in the interest of efficiency to choose as small a space as possible (for a given accuracy), in mixed methods one also often require spaces to be not too large for stability reasons. In the HDG method, stability is guaranteed through the penalty parameters $\tau_{n}$ and $\tau_{t}$. A consequence of this is that (3.8) is uniquely solvable, no matter how large $\left(\boldsymbol{M}_{h}\right)_{t}$ and $\Psi_{h}$ are. For the analogous hybridized mixed method of [8], we needed the trace spaces corresponding to $\left(\boldsymbol{M}_{h}\right)_{t}$ and $\Psi_{h}$ to be exactly equal to certain spaces of jumps, which created additional implementation issues such as construction of local basis functions for the spaces.

3.2. Hybridization of type II. A formulation with velocity and means of pressure. Recalling our scheme for construction of hybridized methods described in the beginning of this section, we now consider the case when $\boldsymbol{u}_{t}$ and $\boldsymbol{u}_{n}$ (i.e., all components of $\boldsymbol{u}$ ) are chosen as the unknowns in the mesh interfaces. Correspondingly, we should use the transmission conditions on the other two variables, namely

$$
\llbracket \boldsymbol{\omega} \times\left.\boldsymbol{n} \rrbracket\right|_{\mathscr{E}_{h}^{\circ}}=0, \quad \text { and }\left.\quad \llbracket p \boldsymbol{n} \rrbracket\right|_{\mathscr{E}_{h}^{\circ}}=0
$$

to derive a hybridized formulation. However, the success of this strategy relies on us being able to compute approximate Stokes solutions within each element locally, once a discrete approximation to $\boldsymbol{u}$, say $\boldsymbol{\lambda}$, is obtained on the boundary of every mesh element. Here we find a difficulty not encountered in the previous case, namely the HDG discretization (2.2) on one element with $\boldsymbol{\lambda}$ as boundary data (of Type II) is not solvable in general, unless

$$
\int_{\partial K} \boldsymbol{\lambda}_{n} \cdot \boldsymbol{n}=0
$$

as seen from Proposition 2.1(2). Thus we are led to modify our local solvers, which in turn necessitates the introduction of a new variable $(\bar{\rho})$ approximating the means of pressures on the element boundaries, as we shall see now. 
The new local solver, denoted by $\mathcal{L}^{\mathrm{II}}$, maps a given function $(\boldsymbol{\lambda}, \bar{\rho}, \boldsymbol{f})$ in $\boldsymbol{L}^{2}\left(\partial \Omega_{h}\right) \times$ $\ell^{2}\left(\partial \Omega_{h}\right) \times \boldsymbol{L}^{2}(\Omega)$ to a triple $(\mathcal{W}, \mathcal{U}, \mathcal{P}) \in \boldsymbol{W}_{h} \times \boldsymbol{V}_{h} \times P_{h}$ defined below. Here, $\ell^{2}\left(\partial \Omega_{h}\right)$ denotes the set of functions in $L^{2}\left(\partial \Omega_{h}\right)$ that are constant on each $\partial K$ for all mesh elements $K$. On any element $K \in \Omega_{h}$, the function $(\mathcal{W}, \mathcal{U}, \mathcal{P})$ restricted to $K$ is in $\boldsymbol{W}(K) \times \boldsymbol{V}(K) \times P(K)$ and satisfies

$$
\begin{array}{rlr}
(\boldsymbol{\mathcal { W }}, \boldsymbol{\tau})_{K}-(\boldsymbol{U}, \operatorname{curl} \boldsymbol{\tau})_{K} & =-\langle\boldsymbol{\lambda}, \boldsymbol{n} \times \boldsymbol{\tau}\rangle_{\partial K}, \\
(\boldsymbol{\mathcal { W }}, \operatorname{curl} \boldsymbol{v})_{K}+\langle\widehat{\mathcal{W}}, \boldsymbol{v} \times \boldsymbol{n}\rangle_{\partial K} & \\
-(\mathcal{P}, \operatorname{div} \boldsymbol{v})_{K}+\langle\widehat{\mathcal{P}}, \boldsymbol{v} \cdot \boldsymbol{n}\rangle_{\partial K} & =(\boldsymbol{f}, \boldsymbol{v})_{K}, \\
-(\boldsymbol{U}, \operatorname{grad} q)_{K} & =\langle\boldsymbol{\lambda} \cdot \boldsymbol{n}, q-\bar{q}\rangle_{\partial K}, \\
\overline{\mathcal{P}} & =\bar{\rho},
\end{array}
$$

where

$$
\begin{aligned}
& \widehat{\mathcal{W}}=\mathcal{W}+\tau_{t}(\mathcal{U}-\boldsymbol{\lambda}) \times \boldsymbol{n}, \\
& \widehat{\mathcal{P}}=\mathcal{P}+\tau_{n}(\boldsymbol{U}-\boldsymbol{\lambda}) \cdot \boldsymbol{n} .
\end{aligned}
$$

Here, we use the convention that for a given function $q$ (that may not be in $\ell^{2}\left(\partial \Omega_{h}\right)$ ), we understand $\bar{q}$ to mean the function in $\ell^{2}\left(\partial \Omega_{h}\right)$ satisfying

$$
\left.\bar{q}\right|_{\partial K}=\frac{1}{|\partial K|} \int_{\partial K} q d \gamma .
$$

Obviously, for functions $\rho$ in $\ell^{2}\left(\partial \Omega_{h}\right)$, we have $\bar{\rho}=\rho$. Let $\boldsymbol{\lambda}_{n}^{0}$ be the function on $\partial \Omega_{h}$ defined by $\left.\boldsymbol{\lambda}_{n}^{0}\right|_{\partial K}=\left.\boldsymbol{\lambda}_{n}\right|_{\partial K}-\left.\overline{\boldsymbol{\lambda} \cdot \boldsymbol{n}}\right|_{\partial K} \boldsymbol{n}$, for all mesh elements $K$. Then, we can rewrite the right hand side of (3.11c) as $\left\langle\boldsymbol{\lambda}_{n}^{0}, q \boldsymbol{n}\right\rangle_{\partial K}$. Hence, the system (3.11) minus (3.11d) is the same as the HDG system (2.2) applied to one element with the data $\boldsymbol{g}_{t}=\boldsymbol{\lambda}_{t}$ and $\boldsymbol{g}_{n}=\boldsymbol{\lambda}_{n}^{0}$. Consequently, by Proposition 2.1(2), the system has a solution, and moreover, the solution is unique once (3.11d) is added to the system. Thus, the map $\mathcal{L}^{\text {II }}$ is well defined.

Note that (3.11) is the HDG discretization of the exact Stokes problem

$$
\begin{aligned}
\boldsymbol{\omega}_{K}-\operatorname{curl} \boldsymbol{u}_{K} & =0 & & \text { in } K, \\
\operatorname{curl} \boldsymbol{\omega}_{K}+\operatorname{grad} p_{K} & =\boldsymbol{f} & & \text { in } K, \\
\operatorname{div} \boldsymbol{u}_{K} & =0 & & \text { in } K, \\
\boldsymbol{u}_{K} & =\boldsymbol{\lambda}_{t}+\boldsymbol{\lambda}_{n}^{0} & & \text { on } \partial K, \\
\bar{p}_{K} & =\bar{\rho}, & &
\end{aligned}
$$

on a single element $K$.

Next, we find conditions on $(\boldsymbol{\lambda}, \bar{\rho}, \boldsymbol{f})$ that makes $(\mathcal{W}, \mathcal{U}, \mathcal{P}) \equiv \mathcal{L}^{\mathrm{II}}(\boldsymbol{\lambda}, \bar{\rho}, \boldsymbol{f})$ equal to $\left(\boldsymbol{\omega}_{h}, \boldsymbol{u}_{h}, p_{h}\right)$. First, we restrict $\boldsymbol{\lambda}$ to the space $\boldsymbol{M}_{h}$ defined by

$$
\begin{aligned}
\boldsymbol{M}_{h} & :=\left\{\boldsymbol{\mu} \in \boldsymbol{L}^{2}\left(\mathscr{E}_{h}\right):\left.\quad \boldsymbol{\mu}\right|_{e} \in \boldsymbol{M}(e) \quad \forall e \in \mathscr{E}_{h}\right\}, \\
\bar{\Psi}_{h} & :=\ell^{2}\left(\partial \Omega_{h}\right),
\end{aligned}
$$

where $\boldsymbol{M}(e)$ is a finite dimensional space on the face $e \in \mathscr{E}_{h}$ such that

$$
\begin{array}{r}
\boldsymbol{M}(e) \supseteq\left\{\left.(\boldsymbol{v}+\boldsymbol{n} \times \boldsymbol{\tau}+\boldsymbol{n} q)\right|_{e}:(\boldsymbol{\tau}, \boldsymbol{v}, q) \in \boldsymbol{W}(K) \times \boldsymbol{V}(K) \times P(K)\right. \\
\forall K: e \subset \partial K\} .
\end{array}
$$


Then we have the following theorem, which identifies certain discrete analogues of (3.9) as sufficient conditions for the coincidence of the locally recovered solution with the HDG solution.

Theorem 3.3 (Conditions for type II hybridization). Suppose $\left(\boldsymbol{\omega}_{h}, \boldsymbol{u}_{h}, p_{h}\right)$ is the solution of the HDG method defined by (2.2), (2.5), and (2.6). Assume that $(\boldsymbol{\lambda}, \bar{\rho}) \in \boldsymbol{M}_{h} \times \bar{\Psi}_{h}$ is such that

$$
\begin{array}{rlrl}
\boldsymbol{\lambda} & =\boldsymbol{g} & & \text { on } \partial \Omega, \\
\left\langle\llbracket \boldsymbol{n} \times \widehat{\mathcal{W}} \rrbracket, \boldsymbol{\mu}_{t}\right\rangle_{\mathscr{E}_{h}}=0 & & \forall \boldsymbol{\mu} \in \boldsymbol{M}_{h}, \\
\left\langle\llbracket \widehat{\mathcal{P}} \boldsymbol{n} \rrbracket, \boldsymbol{\mu}_{n}\right\rangle_{\mathscr{E}_{h}}=0 & & \forall \boldsymbol{\mu} \in \boldsymbol{M}_{h}, \\
\langle\boldsymbol{\lambda} \cdot \boldsymbol{n}, \bar{q}\rangle_{\partial \Omega_{h}}=0 & & \forall \bar{q} \in \bar{\Psi}_{h}, \\
(\mathcal{P}, 1)_{\Omega} & =0 . & &
\end{array}
$$

Then $(\mathcal{W}, \mathcal{U}, \mathcal{P})=\left(\boldsymbol{\omega}_{h}, \boldsymbol{u}_{h}, p_{h}\right), \boldsymbol{\lambda}_{t}=\left(\widehat{\boldsymbol{u}}_{h}\right)_{t}$, and $\boldsymbol{\lambda}_{n}=\left(\widehat{\boldsymbol{u}}_{h}\right)_{n}$.

Proof. We will show that $(\mathcal{W}, \mathcal{U}, \mathcal{P})$ and $\left(\boldsymbol{\omega}_{h}, \boldsymbol{u}_{h}, p_{h}\right)$ satisfy the same set of equations. To do this, just as in the proof of Theorem 3.1, it suffices to show that the numerical traces $(\widehat{\mathcal{W}})_{t}, \boldsymbol{\lambda}_{t}, \boldsymbol{\lambda}_{n}$, and $\widehat{\mathcal{P}}$ can be related to $(\mathcal{W}, \mathcal{U}, \mathcal{P})$ through the expressions in (2.5).

We therefore derive expressions for $(\widehat{\mathcal{W}})_{t}, \boldsymbol{\lambda}_{t}, \boldsymbol{\lambda}_{n}$, and $\widehat{\mathcal{P}}$. By the choice of the space $\boldsymbol{M}_{h}$, the jump conditions (3.15b) and (3.15c) imply that

$$
\llbracket \boldsymbol{n} \times \widehat{\mathcal{W}} \rrbracket=0 \quad \text { and } \quad \llbracket \widehat{\mathcal{P}} \boldsymbol{n} \rrbracket=\mathbf{0} \quad \text { on } \mathscr{E}_{h}^{o} .
$$

Inserting the definition of the numerical traces (3.11e) and (3.11f), we readily obtain that, on $\mathscr{E}_{h}$,

$$
\begin{array}{r}
\llbracket \boldsymbol{n} \times \mathcal{W} \rrbracket+\tau_{t}^{+}\left(\mathfrak{U}^{+}\right)_{t}+\tau_{t}^{-}\left(\mathfrak{U}^{-}\right)_{t}-\left(\tau_{t}^{+}+\tau_{t}^{-}\right) \boldsymbol{\lambda}_{t}=0, \\
\llbracket \mathcal{P} \boldsymbol{n} \rrbracket+\tau_{n}^{+}\left(\mathcal{U}^{+}\right)_{n}+\tau_{n}^{-}\left(\mathcal{U}^{-}\right)_{n}-\left(\tau_{n}^{+}+\tau_{n}^{-}\right) \boldsymbol{\lambda}_{n}=0,
\end{array}
$$

or equivalently,

$$
\begin{aligned}
\boldsymbol{\lambda}_{t} & =\left(\frac{\tau_{t}^{+}\left(\mathfrak{U}^{+}\right)_{t}+\tau_{t}^{-}\left(\mathfrak{U}^{-}\right)_{t}}{\tau_{t}^{-}+\tau_{t}^{+}}\right)+\left(\frac{1}{\tau_{t}^{-}+\tau_{t}^{+}}\right) \llbracket \boldsymbol{n} \times \mathfrak{W} \rrbracket, \\
\boldsymbol{\lambda}_{n} & =\left(\frac{\tau_{t}^{+}\left(\mathfrak{U}^{+}\right)_{n}+\tau_{t}^{-}\left(\mathfrak{U}^{-}\right)_{n}}{\tau_{t}^{-}+\tau_{t}^{+}}\right)+\left(\frac{1}{\tau_{t}^{-}+\tau_{t}^{+}}\right) \llbracket \mathcal{P} \boldsymbol{n} \rrbracket .
\end{aligned}
$$

Hence,

$$
\begin{aligned}
(\widehat{\mathcal{W}})_{t} & =\left(\frac{\tau_{t}^{-}\left(\mathcal{W}^{+}\right)_{t}+\tau_{t}^{+}\left(\mathcal{W}^{-}\right)_{t}}{\tau_{t}^{-}+\tau_{t}^{+}}\right)+\left(\frac{\tau_{t}^{+} \tau_{t}^{-}}{\tau_{t}^{-}+\tau_{t}^{+}}\right) \llbracket \mathfrak{U} \times \boldsymbol{n} \rrbracket \\
\widehat{\mathcal{P}} & =\left(\frac{\tau_{n}^{+} \mathcal{P}^{+}+\tau_{n}^{-} \mathcal{P}^{-}}{\tau_{n}^{-}+\tau_{n}^{+}}\right)+\left(\frac{\tau_{n}^{+} \tau_{n}^{-}}{\tau_{n}^{-}+\tau_{n}^{+}}\right) \llbracket \mathfrak{U} \cdot \boldsymbol{n} \rrbracket .
\end{aligned}
$$

In other words, the numerical traces satisfy the equations $(2.5 \mathrm{a}),(2.5 \mathrm{~b}),(2.5 \mathrm{c})$ and (2.5d). The fact that they also satisfy (2.6) follows from conditions (3.15a) and (3.15c) and the definition of the local solvers.

Consequently, by Proposition 2.1(2), we conclude that the difference between $(\mathcal{W}, \mathcal{U}, \mathcal{P})$ and $\left(\boldsymbol{\omega}_{h}, \boldsymbol{u}_{h}, p_{h}\right)$ is $(\mathbf{0}, \mathbf{0}, \kappa)$ for some constant function $\kappa$. Equation (3.15e) then completes the proof. $\square$ 
Next, we show that the jump conditions (3.15b) and (3.15c) define a mixed method for the velocity traces and pressure averages on element boundaries. We denote by $\boldsymbol{M}_{h}^{o}$ the set of functions in $\boldsymbol{M}_{h}$ that vanish on $\partial \Omega$ and split $\boldsymbol{\lambda}=\boldsymbol{\lambda}^{o}+\boldsymbol{g}$ with $\boldsymbol{\lambda}^{o}$ in $\boldsymbol{M}_{h}^{o}$. In analogy with (3.7) of the type I hybridization, we now define the specific local solutions for this case by

$$
\begin{aligned}
\left(\mathcal{W}_{\boldsymbol{\lambda}}, \mathcal{U}_{\boldsymbol{\lambda}}, \mathcal{P}_{\boldsymbol{\lambda}}\right) & :=\mathcal{L}^{\mathrm{II}}(\boldsymbol{\lambda}, 0, \mathbf{0}), \\
\left(\mathcal{W}_{\bar{\rho}}, \mathcal{U}_{\bar{\rho}}, \mathcal{P}_{\bar{\rho}}\right) & :=\mathcal{L}^{\mathrm{II}}(\mathbf{0}, \bar{\rho}, \mathbf{0}), \\
\left(\mathcal{W}_{\boldsymbol{f}}, \mathcal{U}_{\boldsymbol{f}}, \mathcal{P}_{\boldsymbol{f}}\right) & :=\mathcal{L}^{\mathrm{II}}(\mathbf{0}, 0, \boldsymbol{f}),
\end{aligned}
$$

but note that by Proposition 2.1(2),

$$
\left(\mathcal{W}_{\bar{\rho}}, \mathcal{U}_{\bar{\rho}}, \mathcal{P}_{\bar{\rho}}\right)=(\mathbf{0}, \mathbf{0}, \bar{\rho})
$$

Our main result for the type II hybridization is the following theorem.

THEOREM 3.4 (Characterization of the approximate solution). We have that

$$
\begin{aligned}
& \boldsymbol{\omega}_{h}=\mathcal{W}_{\boldsymbol{\lambda}^{\circ}}+\mathcal{W}_{\boldsymbol{f}}+\mathcal{W}_{\boldsymbol{g}}, \\
& \boldsymbol{u}_{h}=\mathcal{U}_{\boldsymbol{\lambda}^{\circ}}+\mathcal{U}_{\boldsymbol{f}}+\mathcal{U}_{\boldsymbol{g}}, \\
& p_{h}=\mathcal{P}_{\boldsymbol{\lambda}^{\circ}}+\mathcal{P}_{\boldsymbol{f}}+\mathcal{P}_{\boldsymbol{g}}+\mathcal{P}_{\bar{\rho}},
\end{aligned}
$$

where $\left(\boldsymbol{\lambda}^{o}, \bar{\rho}\right)$ is the only element of $\boldsymbol{M}_{h}^{o} \times \bar{\Psi}_{h}$ such that

$$
\begin{aligned}
a_{h}\left(\boldsymbol{\lambda}^{o}, \boldsymbol{\mu}\right)+b_{h}(\bar{\rho}, \boldsymbol{\mu}) & =\ell(\boldsymbol{\mu}), \\
-b_{h}\left(\bar{\psi}, \boldsymbol{\lambda}^{o}\right) & =0,
\end{aligned}
$$

for all $(\boldsymbol{\mu}, \bar{\psi}) \in M_{h}^{o} \times \bar{\Psi}_{h}$, and

$$
\left(\mathcal{P}_{\boldsymbol{\lambda}^{o}}+\mathcal{P}_{\bar{\rho}}+\mathcal{P}_{\boldsymbol{f}}+\mathcal{P}_{\boldsymbol{g}}, 1\right)_{\Omega}=0 .
$$

Here

$$
\begin{aligned}
& a_{h}(\boldsymbol{\lambda}, \boldsymbol{\mu})=\left(\mathcal{W}_{\boldsymbol{\lambda}}, \mathcal{W}_{\boldsymbol{\mu}}\right)_{\Omega_{h}}+\left\langle\tau_{t}\left(\boldsymbol{\lambda}-\boldsymbol{U}_{\boldsymbol{\lambda}}\right)_{t},\left(\boldsymbol{\mu}-\boldsymbol{U}_{\boldsymbol{\mu}}\right)_{t}\right\rangle_{\partial \Omega_{h}} \\
&+\left\langle\tau_{n}\left(\boldsymbol{\lambda}-\boldsymbol{U}_{\boldsymbol{\lambda}}\right)_{n},\left(\boldsymbol{\mu}-\boldsymbol{U}_{\boldsymbol{\mu}}\right)_{n}\right\rangle_{\partial \Omega_{h}}, \\
& b_{h}(\bar{\rho}, \boldsymbol{\mu})=-\langle\bar{\rho}, \boldsymbol{\mu} \cdot \boldsymbol{n}\rangle_{\partial \Omega_{h}}, \\
& \ell(\boldsymbol{\mu})=\left(\boldsymbol{f}, \boldsymbol{U}_{\boldsymbol{\mu}}\right)_{\Omega_{h}}-a_{h}(\boldsymbol{g}, \boldsymbol{\mu}) .
\end{aligned}
$$

A proof can be found in Section 4. For appropriate choice of polynomial spaces, as in the previous case, to satisfy the conditions of Proposition 2.1, we choose the degrees $d_{P}, d_{V}$ and $d_{W}$ to be integers obeying (2.12). Then $\boldsymbol{M}_{h}$ is fixed once we pick any $\boldsymbol{M}(e)$ satisfying (3.14c), e.g., $\boldsymbol{M}(e)=\mathcal{P}_{\max \left(d_{V}, d_{W}, d_{P}\right)}(e)$.

3.3. Hybridization of type III. A formulation with tangential vorticity, normal velocity, and pressure means. Next we hybridize the HDG methods by making another choice of two variables in (3.2), namely $\boldsymbol{\omega}_{t}$ and $\boldsymbol{u}_{n}$, as the unknowns on the mesh interfaces. Their discrete approximations will be denoted by $\gamma_{t}$ and $\boldsymbol{\lambda}_{n}$, respectively. When we try to formulate a system for these unknowns using the transmission conditions on the other two variables, namely

$$
\llbracket \boldsymbol{u} \times\left.\boldsymbol{n} \rrbracket\right|_{\mathscr{E}_{h}^{\circ}}=0, \quad \text { and }\left.\quad \llbracket p \boldsymbol{n} \rrbracket\right|_{\mathscr{E}_{h}^{\circ}}=0
$$


we again face the same difficulty we faced in the Type II case. Consequently, as we shall see, we must introduce a new variable $\bar{\rho}$ approximating the averages of pressure on element boundaries, just as in the Type II case.

To hybridize the HDG method, we begin as in the previous cases by introducing discrete local solutions. These will be obtained using the HDG discretization of the following Stokes problem

$$
\begin{aligned}
\boldsymbol{\omega}_{K}-\operatorname{curl} \boldsymbol{u}_{K} & =0 & & \text { in } K, \\
\operatorname{curl} \boldsymbol{\omega}_{K}+\operatorname{grad} p_{K} & =\boldsymbol{f} & & \text { in } K, \\
\operatorname{div} \boldsymbol{u}_{K} & =0 & & \text { in } K, \\
\left(\boldsymbol{\omega}_{K}\right)_{t} & =\gamma_{t} & & \text { on } \partial K, \\
\left(\boldsymbol{u}_{K}\right)_{n} & =\boldsymbol{\lambda}_{n}^{0} & & \text { on } \partial K, \\
\bar{p}_{K} & =\bar{\rho} . & &
\end{aligned}
$$

on a single element $K$. Given the function $\left(\boldsymbol{\gamma}_{t}, \boldsymbol{\lambda}_{n}, \bar{\rho}, \boldsymbol{f}\right)$ in $\boldsymbol{L}^{2}\left(\partial \Omega_{h}\right) \times L^{2}\left(\partial \Omega_{h}\right) \times$ $\ell^{2}\left(\partial \Omega_{h}\right) \times \boldsymbol{L}^{2}(\Omega)$, we define $(\mathcal{W}, \mathcal{U}, \mathcal{P})$ in $\boldsymbol{W}_{h} \times \boldsymbol{V}_{h} \times P_{h}$ on the element $K \in \Omega_{h}$ as the function in $\boldsymbol{W}(K) \times \boldsymbol{V}(K) \times P(K)$ such that

$$
\begin{aligned}
(\mathcal{W}, \boldsymbol{\tau})_{K}-(\mathcal{U}, \operatorname{curl} \boldsymbol{\tau})_{K}+\langle\widehat{\mathcal{U}}, \boldsymbol{n} \times \boldsymbol{\tau}\rangle_{\partial K} & =0, \\
(\mathcal{W}, \operatorname{curl} \boldsymbol{v})_{K}-(\mathcal{P}, \operatorname{div} \boldsymbol{v})_{K}+\langle\widehat{\mathcal{P}}, \boldsymbol{v} \cdot \boldsymbol{n}\rangle_{\partial K} & =(\boldsymbol{f}, \boldsymbol{v})_{K}-\left\langle\boldsymbol{\gamma}_{t}, \boldsymbol{v} \times \boldsymbol{n}\right\rangle_{\partial K}, \\
-(\mathcal{U}, \operatorname{grad} q)_{K} & =-\left\langle\boldsymbol{\lambda}_{n} \cdot \boldsymbol{n}, q-\bar{q}\right\rangle_{\partial K} . \\
\overline{\mathcal{P}} & =\bar{\rho},
\end{aligned}
$$

where

$$
\begin{gathered}
\widehat{\mathfrak{U}}=\mathfrak{U}+\frac{1}{\tau_{t}} \boldsymbol{n} \times\left(\mathcal{W}-\gamma_{t}\right), \\
\widehat{\mathcal{P}}=\mathcal{P}+\tau_{n}\left(\mathcal{U}-\boldsymbol{\lambda}_{n}\right) \cdot \boldsymbol{n} .
\end{gathered}
$$

By Proposition 2.1(3), there is a unique solution to (3.20) on each mesh element $K$. In other words, the local solver $\mathcal{L}^{\text {III }}\left(\gamma_{t}, \boldsymbol{\lambda}_{n}, \bar{\rho}, \boldsymbol{f}\right):=(\mathcal{W}, \mathcal{U}, \mathcal{P})$ is well defined.

As in the previous cases, we now proceed to identify the discrete analogues of (3.18) that make $\mathcal{L}^{\text {III }}\left(\boldsymbol{\gamma}_{t}, \boldsymbol{\lambda}_{n}, \bar{\rho}, \boldsymbol{f}\right)$ identical to $\left(\boldsymbol{\omega}_{h}, \boldsymbol{u}_{h}, p_{h}\right)$. This will yield a mixed method for $\left(\gamma_{t}, \boldsymbol{\lambda}_{n}, \bar{\rho}, \boldsymbol{f}\right)$. To do this, we begin by restricting the function $\left(\boldsymbol{\gamma}_{t}, \boldsymbol{\lambda}_{n}, \bar{\rho}\right)$ to the space $\left(\boldsymbol{G}_{h}\right)_{t} \times\left(\boldsymbol{M}_{h}\right)_{n} \times \bar{\Psi}_{h}$, where

$$
\begin{aligned}
\left(\boldsymbol{G}_{h}\right)_{t}:=\left\{\boldsymbol{\delta}_{t} \in \boldsymbol{L}^{2}\left(\mathscr{E}_{h}\right):\right. & \left.\left.\boldsymbol{\delta}_{t}\right|_{e} \in \boldsymbol{G}(e) \quad \forall e \in \mathscr{E}_{h}\right\}, \\
\left(\boldsymbol{M}_{h}\right)_{n}:=\left\{\boldsymbol{\mu}_{n} \in \boldsymbol{L}^{2}\left(\mathscr{E}_{h}\right):\right. & \left.\left.\boldsymbol{\mu}_{n}\right|_{e} \in \boldsymbol{M}(e) \quad \forall e \in \mathscr{E}_{h}\right\}, \\
\bar{\Psi}_{h}:=\left\{\bar{\psi} \in L^{2}\left(\partial \Omega_{h}\right):\right. & \left.\left.\bar{\psi}\right|_{\partial K} \in \mathbb{R} \quad \forall K \in \Omega_{h}\right\} \equiv \ell^{2}\left(\partial \Omega_{h}\right),
\end{aligned}
$$

where, $\boldsymbol{G}(e)$ and $\boldsymbol{M}(e)$ for each face $e \in \mathscr{E}_{h}$ are finite dimensional spaces satisfying

$$
\begin{aligned}
\boldsymbol{G}(e) & \supseteq\left\{\left.\left(\boldsymbol{v}_{t}+\boldsymbol{n} \times \boldsymbol{\tau}\right)\right|_{e}:(\boldsymbol{\tau}, \boldsymbol{v}) \in \boldsymbol{W}(K) \times \boldsymbol{U}(K) \quad \forall K: e \subset \partial K\right\}, \\
\boldsymbol{M}(e) & \supseteq\left\{\left.\left(\boldsymbol{v}_{n}+\boldsymbol{n} q\right)\right|_{e}:(\boldsymbol{v}, q) \in \boldsymbol{U}(K) \times P(K) \quad \forall K: e \subset \partial K\right\},
\end{aligned}
$$

Theorem 3.5 (Conditions for type III hybridization). Suppose $\left(\boldsymbol{\omega}_{h}, \boldsymbol{u}_{h}, p_{h}\right)$ is the solution of the HDG method defined by (2.2), (2.5), and (2.6). Assume that 
$\left(\boldsymbol{\gamma}_{t}, \boldsymbol{\lambda}_{n}, \bar{\rho}\right) \in\left(\boldsymbol{G}_{h}\right)_{t} \times\left(\boldsymbol{M}_{h}\right)_{n} \times \bar{\Psi}_{h}$, is such that

$$
\begin{aligned}
\boldsymbol{\lambda}_{n} & =\boldsymbol{g}_{n} & & \text { on } \partial \Omega, \\
\left\langle\llbracket \widehat{\mathcal{U}} \times \boldsymbol{n} \rrbracket, \boldsymbol{\delta}_{t}\right\rangle_{\mathscr{E}_{h}} & =\left\langle\boldsymbol{g}_{t} \times \boldsymbol{n}, \boldsymbol{\delta}_{t}\right\rangle_{\partial \Omega} & & \forall \boldsymbol{\delta}_{t} \in\left(\boldsymbol{G}_{h}\right)_{t}, \\
\left\langle\llbracket \widehat{\mathcal{P}} \boldsymbol{n} \rrbracket, \boldsymbol{\mu}_{n}\right\rangle_{\mathscr{E}_{h}} & =0 & & \forall \boldsymbol{\mu}_{n} \in\left(\boldsymbol{M}_{h}\right)_{n}, \\
\left\langle\boldsymbol{\lambda}_{n} \cdot \boldsymbol{n}, \bar{q}\right\rangle_{\partial \Omega_{h}} & =0 & & \forall \bar{q} \in \bar{\Psi}_{h}, \\
(\mathcal{P}, 1)_{\Omega} & =0 . & &
\end{aligned}
$$

Then $(\mathcal{W}, \mathcal{u}, \mathcal{P})=\left(\boldsymbol{\omega}_{h}, \boldsymbol{u}_{h}, p_{h}\right), \boldsymbol{\lambda}_{n}=\left(\widehat{\boldsymbol{u}}_{h}\right)_{n}$, and $\boldsymbol{\gamma}_{t}=\left(\widehat{\boldsymbol{\omega}}_{h}\right)_{t}$.

Proof. We begin by noting that $(\mathcal{W}, \mathcal{U}, \mathcal{P})$ is in the space $\boldsymbol{W}_{h} \times \boldsymbol{V}_{h} \times P_{h}$. Moreover, $(\mathcal{W}, \mathfrak{U}, \mathcal{P})$ satisfies the weak formulation $(2.2)$ by the definition of the local solver (3.20).

Next, we note that, by the choice of the space $\left(\boldsymbol{G}_{h}\right)_{t} \times\left(\boldsymbol{M}_{h}\right)_{n}$, the jump conditions (3.22b) and (3.22c) imply that

$$
\llbracket \widehat{\mathcal{U}} \times \boldsymbol{n} \rrbracket=0 \quad \text { and } \quad \llbracket \widehat{\mathcal{P}} \boldsymbol{n} \rrbracket=0 \quad \text { on } \mathscr{E}_{h} .
$$

Inserting the definition of the numerical traces (3.20e) and (3.20f), we readily obtain that, on $\mathscr{E}_{h}^{o}$,

$$
\begin{array}{r}
\llbracket \mathcal{U} \times \boldsymbol{n} \rrbracket+\frac{1}{\tau_{t}^{+}}\left(\mathcal{W}^{+}\right)_{t}+\frac{1}{\tau_{t}^{-}}\left(\mathcal{W}^{-}\right)_{t}-\left(\frac{1}{\tau_{t}^{+}}+\frac{1}{\tau_{t}^{-}}\right) \boldsymbol{\gamma}_{t}=0, \\
\llbracket \mathcal{P} \boldsymbol{n} \rrbracket+\tau_{n}^{+}\left(\mathfrak{U}_{h}^{+}\right)_{n}+\tau_{n}^{-}\left(\mathcal{U}_{h}^{-}\right)_{n}-\left(\tau_{n}^{+}+\tau_{n}^{-}\right) \boldsymbol{\lambda}_{n}=0,
\end{array}
$$

or, equivalently,

$$
\begin{aligned}
\boldsymbol{\gamma}_{t} & =\left(\frac{\tau_{t}^{-}\left(\mathcal{W}^{+}\right)_{t}+\tau_{t}^{+}\left(\mathcal{W}^{-}\right)_{t}}{\tau_{t}^{-}+\tau_{t}^{+}}\right)+\left(\frac{\tau_{t}^{-} \tau_{t}^{+}}{\tau_{t}^{-}+\tau_{t}^{+}}\right) \llbracket \mathcal{U} \times \boldsymbol{n} \rrbracket, \\
\boldsymbol{\lambda}_{n} & =\left(\frac{\tau_{n}^{+}\left(\mathfrak{U}^{+}\right)_{n}+\tau_{n}^{-}\left(\mathfrak{U}^{-}\right)_{n}}{\tau_{n}^{-}+\tau_{n}^{+}}\right)+\left(\frac{1}{\tau_{n}^{-}+\tau_{n}^{+}}\right) \llbracket \mathcal{P} \boldsymbol{n} \rrbracket .
\end{aligned}
$$

Hence,

$$
\begin{aligned}
(\widehat{\mathfrak{u}})_{t} & =\left(\frac{\tau_{t}^{+}\left(\mathfrak{u}^{+}\right)_{t}+\tau_{t}^{-}\left(\mathfrak{U}^{-}\right)_{t}}{\tau_{t}^{-}+\tau_{t}^{+}}\right)+\left(\frac{1}{\tau_{t}^{-}+\tau_{t}^{+}}\right) \llbracket \boldsymbol{n} \times \mathcal{W} \rrbracket, \\
\widehat{\mathcal{P}} & =\left(\frac{\tau_{n}^{-} \mathcal{P}^{+}+\tau_{n}^{+} \mathcal{P}^{-}}{\tau_{n}^{-}+\tau_{n}^{+}}\right)+\left(\frac{\tau_{n}^{-} \tau_{n}^{+}}{\tau_{n}^{-}+\tau_{n}^{+}}\right) \llbracket \mathfrak{U} \cdot \boldsymbol{n} \rrbracket .
\end{aligned}
$$

In other words, the numerical traces satisfy the equations $(2.5 \mathrm{a}),(2.5 \mathrm{~b}),(2.5 \mathrm{c})$ and $(2.5 \mathrm{~d})$. The fact that they also satisfy (2.6) follows from conditions (3.22a) and (3.22c). They also satisfy the equations (2.6c) and (2.6d) by definition of the local solvers.

By the uniqueness result of Proposition 2.1(2), we can now conclude that the approximation $(\mathcal{W}, \mathcal{U}, \mathcal{P})$ coincides with $\left(\boldsymbol{\omega}_{h}, \boldsymbol{u}_{h}, p_{h}\right)$. Moreover, we also have $\boldsymbol{\gamma}_{t}=$ $\left(\widehat{\boldsymbol{\omega}}_{h}\right)_{t}$ and $\boldsymbol{\lambda}_{n}=\left(\widehat{\boldsymbol{u}}_{h}\right)_{n}$. This completes the proof. $\mathrm{Q}$

We now proceed to formulate a mixed method for the numerical traces. Define specific local solutions by

$$
\begin{array}{rlrl}
\left(\mathcal{W}_{\boldsymbol{\gamma}_{t}}, \mathcal{U}_{\boldsymbol{\gamma}_{t},}, \mathcal{P}_{\boldsymbol{\gamma}_{t}}\right) & :=\mathcal{L}^{\mathrm{III}}\left(\boldsymbol{\gamma}_{t}, \mathbf{0}, 0, \mathbf{0}\right), & \left(\mathcal{W}_{\boldsymbol{\lambda}_{n}}, \mathcal{U}_{\boldsymbol{\lambda}_{n},}, \mathcal{P}_{\boldsymbol{\lambda}_{n}}\right):=\mathcal{L}^{\mathrm{III}}\left(\mathbf{0}, \boldsymbol{\lambda}_{n}, 0, \mathbf{0}\right), \\
\left(\mathcal{W}_{\bar{\rho}}, \mathcal{U}_{\bar{\rho}}, \mathcal{P}_{\bar{\rho}}\right):=\mathcal{L}^{\mathrm{III}}(\mathbf{0}, \mathbf{0}, \bar{\rho}, \mathbf{0}), & \left(\mathcal{W}_{\boldsymbol{f}}, \mathcal{U}_{\boldsymbol{f}}, \mathcal{P}_{\boldsymbol{f}}\right):=\mathcal{L}^{\mathrm{III}}(\mathbf{0}, \mathbf{0}, 0, \boldsymbol{f}),
\end{array}
$$


and observe that by Proposition 2.1(2), $\left(\mathcal{W}_{\bar{\rho}}, \mathfrak{U}_{\bar{\rho}}, \mathcal{P}_{\bar{\rho}}\right)=(\mathbf{0}, \mathbf{0}, \bar{\rho})$. We additionally denote by $\left(\boldsymbol{M}_{h}^{o}\right)_{n}$ the functions of $\left(\boldsymbol{M}_{h}\right)_{n}$ which are zero on $\partial \Omega$, and we write $\boldsymbol{\lambda}_{n}$ as the sum of $\boldsymbol{\lambda}_{n}^{o}$ and $\boldsymbol{g}_{n}$, where $\boldsymbol{\lambda}_{n}^{o}$ is in $\left(\boldsymbol{M}_{h}^{o}\right)_{n}$. We are now ready to state our main result.

THEOREM 3.6 (Characterization of the approximate solution). We have that

$$
\begin{aligned}
& \boldsymbol{\omega}_{h}=\mathcal{W}_{\gamma_{t}}+\mathcal{W}_{\boldsymbol{\lambda}_{n}^{o}}+\mathcal{W}_{\boldsymbol{f}}+\mathcal{W}_{\boldsymbol{g}_{n},}, \\
& \boldsymbol{u}_{h}=\mathcal{U}_{\gamma_{t}}+\mathcal{U}_{\boldsymbol{\lambda}_{n}^{o}}+\mathcal{U}_{\boldsymbol{f}}+\mathcal{U}_{\boldsymbol{g}_{n}}, \\
& p_{h}=\mathcal{P}_{\boldsymbol{\gamma}_{t}}+\mathcal{P}_{\boldsymbol{\lambda}_{n}^{o}}+\mathcal{P}_{\boldsymbol{f}}+\mathcal{P}_{\boldsymbol{g}_{n}}+\mathcal{P}_{\bar{\rho}},
\end{aligned}
$$

where $\left(\boldsymbol{\gamma}_{t}, \boldsymbol{\lambda}_{n}^{o}, \bar{\rho}\right)$ is the only element of $\left(\boldsymbol{G}_{h}\right)_{t} \times\left(\boldsymbol{M}_{h}^{o}\right)_{n} \times \bar{\Psi}_{h}$ such that

$$
\begin{aligned}
a_{h}\left(\boldsymbol{\gamma}_{t}, \boldsymbol{\delta}_{t}\right)+b_{h}\left(\boldsymbol{\lambda}_{n}, \boldsymbol{\delta}_{t}\right) & =\ell_{1}\left(\boldsymbol{\delta}_{t}\right), \\
-b_{h}\left(\boldsymbol{\mu}_{n}, \boldsymbol{\gamma}_{t}\right)+c_{h}\left(\boldsymbol{\lambda}_{n}, \boldsymbol{\mu}_{n}\right)+d_{h}\left(\bar{\rho}, \boldsymbol{\mu}_{n}\right) & =\ell_{2}\left(\boldsymbol{\mu}_{n}\right), \\
-d_{h}\left(\bar{q}, \boldsymbol{\lambda}_{n}\right) & =0,
\end{aligned}
$$

for all $\left(\boldsymbol{\delta}_{t}, \boldsymbol{\mu}_{n}, \bar{\rho}\right) \in \boldsymbol{G}_{h} \times\left(\boldsymbol{M}_{h}^{o}\right)_{n} \times \bar{\Psi}_{h}$, and

$$
\left(\mathcal{P}_{\boldsymbol{\lambda}_{t}^{o}}+\mathcal{P}_{\rho}+\mathcal{P}_{\boldsymbol{f}}+\mathcal{P}_{\boldsymbol{g}_{t}}, 1\right)_{\Omega}=0 .
$$

Here

$$
\begin{aligned}
a_{h}\left(\boldsymbol{\gamma}_{t}, \boldsymbol{\delta}_{t}\right):= & \left(\mathcal{W}_{\gamma_{t}}, \mathcal{W}_{\boldsymbol{\delta}_{t}}\right)_{\Omega_{h}} \\
& +\left\langle\frac{1}{\tau_{t}} \boldsymbol{n} \times\left(\boldsymbol{\gamma}_{t}-\mathcal{W}_{\boldsymbol{\gamma}_{t}}\right), \boldsymbol{n} \times\left(\boldsymbol{\delta}_{t}-\mathcal{W}_{\boldsymbol{\delta}_{t}}\right)\right\rangle_{\partial \Omega_{h}}+\left\langle\tau_{n}\left(\mathcal{U}_{\boldsymbol{\gamma}_{t}}\right)_{n},\left(\mathcal{U}_{\boldsymbol{\delta}_{t}}\right)_{n}\right\rangle_{\partial \Omega_{h}}, \\
b_{h}\left(\boldsymbol{\lambda}_{n}, \boldsymbol{\delta}_{t}\right):= & \left\langle\boldsymbol{\lambda}_{n}, \mathcal{P}_{\boldsymbol{\delta}_{t}}+\tau_{n}\left(\boldsymbol{U}_{\boldsymbol{\lambda}_{t}}\right)_{n}\right\rangle_{\partial \Omega_{h}}, \\
c_{h}\left(\boldsymbol{\lambda}_{n}, \boldsymbol{\mu}_{n}\right):= & \left(\mathcal{W}_{\boldsymbol{\lambda}_{n}}, \mathcal{W}_{\boldsymbol{\mu}_{n}}\right)_{\Omega_{h}} \\
& +\left\langle\frac{1}{\tau_{t}} \boldsymbol{n} \times \mathcal{W}_{\boldsymbol{\mu}_{n}}, \boldsymbol{n} \times \mathcal{W}_{\boldsymbol{\lambda}_{n}}\right\rangle_{\partial \Omega_{h}}+\left\langle\tau_{n}\left(\boldsymbol{\mu}_{n}-\boldsymbol{U}_{\boldsymbol{\mu}_{n}}\right)_{n},\left(\boldsymbol{\lambda}_{n}-\boldsymbol{U}_{\boldsymbol{\lambda}_{n}}\right)_{n}\right\rangle_{\partial \Omega_{h}} \\
d_{h}\left(\bar{\rho}, \boldsymbol{\mu}_{n}\right):= & -\left\langle\bar{\rho}, \boldsymbol{\mu}_{n} \cdot \boldsymbol{n}\right\rangle_{\partial \Omega_{h}},
\end{aligned}
$$

and

$$
\begin{aligned}
\ell_{1}\left(\boldsymbol{\delta}_{t}\right) & :=-\left(\boldsymbol{f}, \boldsymbol{U}_{\boldsymbol{\delta}_{t}}\right)_{\Omega_{h}}-b_{h}\left(\boldsymbol{g}_{n}, \boldsymbol{\delta}_{t}\right)-\left\langle\boldsymbol{g}_{t} \times \boldsymbol{n}, \boldsymbol{\delta}_{t}\right\rangle_{\partial \Omega}, \\
\ell_{2}\left(\boldsymbol{\mu}_{n}\right) & :=\left(\boldsymbol{f}, \boldsymbol{U}_{\boldsymbol{\mu}_{n}}\right)_{\Omega_{h}}-c_{h}\left(\boldsymbol{g}_{n}, \boldsymbol{\mu}_{n}\right) .
\end{aligned}
$$

3.4. Hybridization of type IV. A formulation with tangential vorticity, pressure, and harmonic velocity potentials. There is now only one more remaining choice of two variables from in (3.2), namely $\boldsymbol{\omega}_{t}$ and $p$, that we have not investigated yet. This is the type IV case. This case presents additional complications not found in previous three cases. The complications are rooted in the same reason for which we did not consider a "type IV boundary condition" in Section 2.

To explain the difficulty, suppose we are given an approximation $\left(\boldsymbol{\gamma}_{t}, \rho\right)$ to $\left(\boldsymbol{\omega}_{t}, p\right)$ on $\partial \Omega_{h}$. To obtain an approximate solution inside the mesh elements, let us try to define a local solution $(\mathcal{W}, \mathcal{U}, \mathcal{P})$ generated by data $\left(\gamma_{t}, \rho, \boldsymbol{f}\right)$ in $\boldsymbol{L}^{2}\left(\partial \Omega_{h}\right) \times L^{2}\left(\partial \Omega_{h}\right) \times$ $\boldsymbol{L}^{2}(\Omega)$. For this, we would like to use the HDG method applied to one element $K$, with boundary conditions on tangential vorticity and pressure (which would be 
discrete versions of boundary conditions $\boldsymbol{\omega}_{t}=\gamma_{t}$ and $p=\rho$ on $\left.\partial K\right)$. Thus we are led to take $(\mathcal{W}, \mathcal{U}, \mathcal{P})$ as the function in $\boldsymbol{W}(K) \times \boldsymbol{V}(K) \times P(K)$ such that

$$
\begin{aligned}
(\mathcal{W}, \boldsymbol{\tau})_{K}-(\boldsymbol{U}, \operatorname{curl} \boldsymbol{\tau})_{K}+\langle\widehat{\mathcal{u}}, \boldsymbol{n} \times \boldsymbol{\tau}\rangle_{\partial K} & =0 \\
(\boldsymbol{W}, \operatorname{curl} \boldsymbol{v})_{K}-(\mathcal{P}, \operatorname{div} \boldsymbol{v})_{K} & =(\boldsymbol{f}, \boldsymbol{v})_{K} \\
& -\left\langle\boldsymbol{n} \times \boldsymbol{\gamma}_{t}+\rho \boldsymbol{n}, \boldsymbol{v}\right\rangle_{\partial K} \\
-(\boldsymbol{U}, \operatorname{grad} q)_{K}+\langle\widehat{\boldsymbol{u}} \cdot \boldsymbol{n}, q\rangle_{\partial K} & =0
\end{aligned}
$$

with $(\widehat{\mathfrak{U}})_{t}=(\mathfrak{U})_{t}+\tau_{t}^{-1} \boldsymbol{n} \times\left(\mathcal{W}-\gamma_{t}\right)$, and $(\widehat{\mathfrak{U}})_{n}=(\mathfrak{U})_{n}+\tau_{n}^{-1}(\mathcal{P}-\rho) \boldsymbol{n}$. Unfortunately this problem is not solvable in general, which is the same reason we omitted this type of boundary condition in Proposition 2.1.

Nonetheless, upon reviewing the proof of Proposition 2.1 in the case of one element, we find that the null space of the above system is of the form $(\mathcal{W}, \mathcal{U}, \mathcal{P})=$ $(\mathbf{0}, \operatorname{grad} \phi, 0)$ where $\phi$ is in the following local space harmonic velocity potentials:

$$
\Phi(K)=\left\{\xi: \operatorname{grad} \xi \in \boldsymbol{V}(K): \Delta \xi=0 \text { and }(\xi, 1)_{K}=0\right\} .
$$

Hence we can recover unique solvability if the velocity is kept orthogonal to $\Phi(K)$. Keeping this in mind, we are motivated to reformulate the local problems to give a consistent system of equations as follows. Denote the $L^{2}$-projection of $\boldsymbol{v} \in \boldsymbol{V}(K)$ into $\operatorname{grad} \Phi(K)$ by $\operatorname{grad} \phi_{\boldsymbol{v}}$. Given the function $\left(\gamma_{t}, \rho, \phi, \boldsymbol{f}\right)$ in $\boldsymbol{L}^{2}\left(\partial \Omega_{h}\right) \times L^{2}\left(\partial \Omega_{h}\right) \times$ $H^{1}\left(\Omega_{h}\right) \times \boldsymbol{L}^{2}(\Omega)$, we define $(\boldsymbol{W}, \mathcal{U}, \mathcal{P})$ in $\boldsymbol{W}_{h} \times \boldsymbol{V}_{h} \times P_{h}$ on the element $K \in \Omega_{h}$ as the function in $\boldsymbol{W}(K) \times \boldsymbol{V}(K) \times P(K)$ such that

$$
\begin{aligned}
(\mathcal{W}, \boldsymbol{\tau})_{K}-(\boldsymbol{U}, \operatorname{curl} \boldsymbol{\tau})_{K}+\langle\widehat{\mathcal{u}}, \boldsymbol{n} \times \boldsymbol{\tau}\rangle_{\partial K} & =0, \\
(\mathcal{W}, \operatorname{curl} \boldsymbol{v})_{K}-(\mathcal{P}, \operatorname{div} \boldsymbol{v})_{K} & =\left(\boldsymbol{f}, \boldsymbol{v}-\operatorname{grad} \phi_{\boldsymbol{v}}\right)_{K} \\
& -\left\langle\boldsymbol{n} \times \boldsymbol{\gamma}_{t}+\rho \boldsymbol{n}, \boldsymbol{v}-\operatorname{grad} \phi_{\boldsymbol{v}}\right\rangle_{\partial K} \\
-(\mathcal{U}, \operatorname{grad} q)_{K}+\langle\widehat{\boldsymbol{u}} \cdot \boldsymbol{n}, q\rangle_{\partial K} & =0, \\
(\mathcal{U}, \operatorname{grad} \xi)_{K} & =(\operatorname{grad} \phi, \operatorname{grad} \xi)_{K},
\end{aligned}
$$

where

$$
\begin{aligned}
& (\widehat{\mathfrak{U}})_{t}=(\mathfrak{U})_{t}+\frac{1}{\tau_{t}} \boldsymbol{n} \times\left(\mathcal{W}-\gamma_{t}\right), \\
& (\widehat{\mathfrak{U}})_{n}=(\mathfrak{U})_{n}+\frac{1}{\tau_{n}}(\mathcal{P}-\rho) \boldsymbol{n} .
\end{aligned}
$$

A minor modification of the arguments in Proposition 2.1 shows unique solvability of (3.24), hence we can define a fourth local solver $\mathcal{L}^{\mathrm{IV}}: \boldsymbol{L}^{2}\left(\partial \Omega_{h}\right) \times L^{2}\left(\partial \Omega_{h}\right) \times H^{1}\left(\Omega_{h}\right) \times$ $\boldsymbol{L}^{2}(\Omega) \mapsto \boldsymbol{W}(K) \times \boldsymbol{V}(K) \times P(K)$ that takes $\left(\gamma_{t}, \rho, \phi, \boldsymbol{f}\right)$ to $(\mathcal{W}, \mathcal{U}, \mathcal{P})$.

Note that (3.24) is a discretization of the exact Stokes problem

$$
\begin{aligned}
\boldsymbol{\omega}_{K}-\operatorname{curl} \boldsymbol{u}_{K}=0 & \text { in } K, \\
\operatorname{curl} \boldsymbol{\omega}_{K}+\operatorname{grad} p_{K}=\boldsymbol{f} & \text { in } K, \\
\operatorname{div} \boldsymbol{u}_{K}=0 & \text { in } K, \\
\left(\boldsymbol{\omega}_{K}\right)_{t}=\gamma_{t} & \text { on } \partial K, \\
p_{K}=\rho & \text { on } \partial K,
\end{aligned}
$$

with the additional condition that the velocity field $\boldsymbol{u}_{K}$ is $L^{2}$-orthogonal to all gradients of harmonic functions, which is necessary for well-posedness. 
Although we could have considered a global "type IV boundary condition" case in Proposition 2.1 through the addition of an equation like (3.24d), it does not appear to be very useful, because we do not know the data needed for the right hand side. However, we can use type IV boundary conditions locally to hybridize a global problem with type II boundary condition because we already have global solvability for the type II boundary condition case. We only need to ensure that the local problems are solvable, and the reformulation of the local solvers with (3.24d) guarantees it.

Now, we proceed as in the previous cases to identify conditions on $\gamma_{t}, \rho$ and $\phi$ in such a way that $(\mathcal{W}, \mathcal{U}, \mathcal{P})$ is identical to $\left(\boldsymbol{\omega}_{h}, \boldsymbol{u}_{h}, p_{h}\right)$. We begin by restricting the function $\left(\gamma_{t}, \rho, \phi\right)$ to the space $\left(\boldsymbol{G}_{h}\right)_{t} \times \Psi_{h} \times \Phi_{h}$, where

$$
\begin{array}{rlrl}
\left(\boldsymbol{G}_{h}\right)_{t}: & :=\left\{\boldsymbol{\delta}_{t} \in \boldsymbol{L}^{2}\left(\mathscr{E}_{h}\right):\right. & \left.\left.\boldsymbol{\delta}_{t}\right|_{e} \in \boldsymbol{G}(e) \quad \forall e \in \mathscr{E}_{h}\right\}, \\
\Psi_{h}:=\left\{\psi \in L^{2}\left(\mathscr{E}_{h}\right):\right. & \left.\left.\psi\right|_{e} \in \Psi(e) \quad \forall e \in \mathscr{E}_{h}\right\}, \\
\Phi_{h}:=\left\{\xi \in H^{1}\left(\Omega_{h}\right):\right. & \left.\left.\xi\right|_{K} \in \Phi(K) \quad \forall K \in \Omega_{h}\right\},
\end{array}
$$

where, on each face $e \in \mathscr{E}_{h}$, we have finite dimensional spaces $\boldsymbol{G}(e)$ and $\Psi(e)$ satisfying

$$
\begin{aligned}
& \boldsymbol{G}(e) \supseteq\left\{\left.\left(\boldsymbol{v}_{t}+\boldsymbol{n} \times \boldsymbol{\tau}\right)\right|_{e}:(\boldsymbol{\tau}, \boldsymbol{v}) \in \boldsymbol{W}(K) \times \boldsymbol{U}(K) \quad \forall K: e \subset \partial K\right\}, \\
& \Psi(e) \supseteq\left\{\left.(q+\boldsymbol{v} \cdot \boldsymbol{n})\right|_{e}:(\boldsymbol{v}, q) \in \boldsymbol{U}(K) \times P(K) \quad \forall K: e \subset \partial K\right\} .
\end{aligned}
$$

The next theorem identifies the discrete analogues of the transmission conditions

$$
\llbracket \boldsymbol{u} \times\left.\boldsymbol{n} \rrbracket\right|_{\mathscr{E}_{h}^{\circ}}=0,\left.\quad \llbracket \boldsymbol{u} \cdot \boldsymbol{n} \rrbracket\right|_{\mathscr{E}_{h}^{\circ}}=0
$$

that recovers the original solution. An additional condition also appears because of our reformulation of the local solvers.

Theorem 3.7 (Conditions for type IV hybridization). Suppose $\left(\boldsymbol{\omega}_{h}, \boldsymbol{u}_{h}, p_{h}\right)$ is the solution of the HDG method defined by (2.2), (2.5), and (2.6). Assume that $\left(\gamma_{t}, \rho, \phi\right) \in \boldsymbol{M}_{h} \times \Psi_{h} \times \Phi_{h}$ is such that

$$
\begin{aligned}
\left\langle\llbracket \widehat{\mathcal{U}} \times \boldsymbol{n} \rrbracket, \boldsymbol{\delta}_{t}\right\rangle_{\mathscr{E}_{h}} & =\left\langle\boldsymbol{g} \times \boldsymbol{n}, \boldsymbol{\delta}_{t}\right\rangle_{\partial \Omega} & & \forall \boldsymbol{\delta}_{t} \in \boldsymbol{G}_{h}, \\
\langle\llbracket \widehat{\mathcal{u}} \cdot \boldsymbol{n} \rrbracket, \psi\rangle_{\mathscr{E}_{h}} & =\langle\boldsymbol{g} \cdot \boldsymbol{n}, \psi\rangle_{\partial \Omega} & & \forall \psi \in \Psi_{h}, \\
\left\langle\boldsymbol{n} \times \gamma_{t}+\rho \boldsymbol{n}, \operatorname{grad} \xi\right\rangle_{\partial \Omega_{h}} & =(\boldsymbol{f}, \operatorname{grad} \xi)_{\Omega_{h}} & & \forall \xi \in \Phi_{h}, \\
(\mathcal{P}, 1)_{\Omega} & =0 . & &
\end{aligned}
$$

Then $(\mathcal{W}, \mathcal{U}, \mathcal{P})=\left(\boldsymbol{\omega}_{h}, \boldsymbol{u}_{h}, p_{h}\right), \gamma_{t}=\left(\widehat{\boldsymbol{\omega}}_{h}\right)_{t}$, and $\rho=\widehat{p}_{h}$.

Proof. The proof is similar to the analogous proofs in the previous three cases, and begins with the observation that $(\mathcal{W}, \mathcal{U}, \mathcal{P})$ satisfies the weak formulation $(2.2)$ by the definition of the local solver (3.24) and condition (3.27c). Next, the jump conditions (3.27a) and (3.27b) imply that

$$
\llbracket \widehat{\mathcal{U}} \times \boldsymbol{n} \rrbracket=0 \quad \text { and } \quad \llbracket \widehat{\mathcal{U}} \cdot \boldsymbol{n} \rrbracket=0 \quad \text { on } \mathscr{E}_{h} .
$$

Inserting the definition of the numerical traces (3.24e) and (3.24f), we readily obtain that, on $\mathscr{E}_{h}$,

$$
\begin{array}{r}
\llbracket \mathcal{U} \times \boldsymbol{n} \rrbracket+\frac{1}{\tau_{t}^{+}}\left(\mathcal{W}^{+}\right)_{t}+\frac{1}{\tau_{t}^{-}}\left(\mathcal{W}^{-}\right)_{t}-\left(\frac{1}{\tau_{t}^{+}}+\frac{1}{\tau_{t}^{-}}\right) \gamma_{t}=0, \\
\llbracket \mathcal{U} \cdot \boldsymbol{n} \rrbracket+\frac{1}{\tau_{n}^{+}} \mathcal{P}_{h}^{+}+\frac{1}{\tau_{n}^{-}} \mathcal{P}_{h}^{-}-\left(\frac{1}{\tau_{n}^{+}}+\frac{1}{\tau_{n}^{-}}\right) \rho=0,
\end{array}
$$


or, equivalently,

$$
\begin{aligned}
\boldsymbol{\gamma}_{t} & =\left(\frac{\tau_{t}^{-}\left(\mathcal{W}^{+}\right)_{t}+\tau_{t}^{+}\left(\mathcal{W}^{-}\right)_{t}}{\tau_{t}^{-}+\tau_{t}^{+}}\right)+\left(\frac{\tau_{t}^{-} \tau_{t}^{+}}{\tau_{t}^{-}+\tau_{t}^{+}}\right) \llbracket \mathfrak{U} \times \boldsymbol{n} \rrbracket, \\
\rho & =\left(\frac{\tau_{n}^{-} \mathcal{P}^{+}+\tau_{n}^{+} \mathcal{P}^{-}}{\tau_{n}^{-}+\tau_{n}^{+}}\right)+\left(\frac{\tau_{n}^{-} \tau_{n}^{+}}{\tau_{n}^{-}+\tau_{n}^{+}}\right) \llbracket \mathcal{U} \cdot \boldsymbol{n} \rrbracket .
\end{aligned}
$$

Hence,

$$
\begin{aligned}
& (\widehat{\mathfrak{u}})_{t}=\left(\frac{\tau_{t}^{+}\left(\mathfrak{U}^{+}\right)_{t}+\tau_{t}^{+}\left(\mathfrak{u}^{-}\right)_{t}}{\tau_{t}^{-}+\tau_{t}^{+}}\right)+\left(\frac{\tau_{t}^{+} \tau_{t}^{-}}{\tau_{t}^{-}+\tau_{t}^{+}}\right) \llbracket \boldsymbol{n} \times \mathcal{W} \rrbracket \\
& (\widehat{\mathfrak{U}})_{n}=\left(\frac{\tau_{n}^{+}\left(\mathfrak{U}^{+}\right)_{n}+\tau_{n}^{-}\left(\mathfrak{U}^{-}\right)_{n}}{\tau_{n}^{-}+\tau_{n}^{+}}\right)+\left(\frac{1}{\tau_{n}^{-}+\tau_{n}^{+}}\right) \llbracket \mathcal{P} \boldsymbol{n} \rrbracket .
\end{aligned}
$$

In other words, $(\mathcal{W}, \mathcal{U}, \mathcal{P})$ satisfies $(2.2),(2.5)$, and (2.6). By the uniqueness result of Proposition 2.1(2), we can now conclude that the approximation $(\mathcal{W}, \mathcal{U}, \mathcal{P})$ coincides with $\left(\boldsymbol{\omega}_{h}, \boldsymbol{u}_{h}, p_{h}\right)$ and consequently $\boldsymbol{\gamma}_{t}=\left(\widehat{\boldsymbol{\omega}}_{h}\right)_{t}$ and $\rho=\widehat{p}_{h}$. $\mathrm{v}$

Next, we give a characterization of the approximate solution in terms of the local solutions

$$
\begin{array}{rlrl}
\left(\mathcal{W}_{\gamma_{t}}, \mathcal{U}_{\gamma_{t}}, \mathcal{P}_{\boldsymbol{\gamma}_{t}}\right) & :=\mathcal{L}^{\mathrm{IV}}\left(\boldsymbol{\gamma}_{t}, 0,0, \mathbf{0}\right), & \left(\mathcal{W}_{\rho}, \mathcal{U}_{\rho}, \mathcal{P}_{\rho}\right) & :=\mathcal{L}^{\mathrm{IV}}(\mathbf{0}, \rho, 0, \mathbf{0}), \\
\left(\mathcal{W}_{\phi}, \mathcal{U}_{\phi}, \mathcal{P}_{\phi}\right):=\mathcal{L}^{\mathrm{IV}}(\mathbf{0}, 0, \phi, \mathbf{0}), & \left(\mathcal{W}_{\boldsymbol{f}}, \mathcal{U}_{\boldsymbol{f}}, \mathcal{P}_{\boldsymbol{f}}\right):=\mathcal{L}^{\mathrm{IV}}(\mathbf{0}, \mathbf{0}, 0, \boldsymbol{f})
\end{array}
$$

Note that

$$
\left(\mathcal{W}_{\phi}, \mathcal{U}_{\phi}, \mathcal{P}_{\phi}\right)=(\mathbf{0}, \operatorname{grad} \phi, 0)
$$

by direct verification in (3.24). The next theorem gives a mixed problem for the numerical traces $\gamma_{t}, \rho$ together with the volumetric unknown $\phi$. The presence of the variable $\phi$ defined within the elements (and not element boundaries, as in the previous cases) may appear to annul the potential advantages of dimensional reduction brought about by hybridization. However, this is not the case because $\phi$ is completely determined by its values on element boundaries.

Theorem 3.8 (Characterization of the approximate solution). We have that

$$
\begin{aligned}
& \boldsymbol{\omega}_{h}=\mathcal{W}_{\gamma_{t}}+\mathcal{W}_{\rho}+\mathcal{W}_{\boldsymbol{f}}, \\
& \boldsymbol{u}_{h}=\boldsymbol{U}_{\gamma_{t}}+\mathcal{U}_{\rho}+\mathcal{U}_{\boldsymbol{f}}+\operatorname{grad} \phi, \\
& p_{h}=\mathcal{P}_{\boldsymbol{\lambda}_{t}^{o}}+\mathcal{P}_{\rho}+\mathcal{P}_{\boldsymbol{f}},
\end{aligned}
$$

where $\left(\gamma_{t}, \rho, \phi\right)$ is the only element of $\left(\boldsymbol{G}_{h}\right)_{t} \times \Psi_{h} \times \Phi_{h}$ such that

$$
\begin{aligned}
a_{h}\left(\gamma_{t}, \boldsymbol{\delta}_{t}\right)+b_{h}\left(\rho, \boldsymbol{\delta}_{t}\right)+c_{h}\left(\phi, \boldsymbol{\delta}_{t}\right) & =\ell_{1}\left(\boldsymbol{\mu}_{t}\right) \\
b_{h}\left(\psi, \gamma_{t}\right)+d_{h}(\rho, \psi)+e_{h}(\phi, \psi) & =\ell_{2}(\psi), \\
-c_{h}\left(\xi, \gamma_{t}\right)-e_{h}(\xi, \rho) & =\ell_{3}(\xi)
\end{aligned}
$$

for all $\left(\boldsymbol{\delta}_{t}, \psi, \xi\right) \in\left(\boldsymbol{G}_{h}\right)_{t} \times \Psi_{h} \times \Phi_{h}$, and

$$
\left(\mathcal{P}_{\gamma_{t}}+\mathcal{P}_{\rho}+\mathcal{P}_{\boldsymbol{f}}, 1\right)_{\Omega}=0 .
$$


Here

$$
\begin{array}{rll}
a_{h}\left(\boldsymbol{\gamma}_{t}, \boldsymbol{\delta}_{t}\right):= & \left(\mathcal{W}_{\boldsymbol{\gamma}_{t}}, \mathcal{W}_{\boldsymbol{\delta}_{t}}\right)_{\Omega_{h}}+\left\langle\frac{1}{\tau_{n}} \mathcal{P}_{\boldsymbol{\gamma}_{t}}, \mathcal{P}_{\boldsymbol{\delta}_{t}}\right\rangle_{\partial \Omega_{h}} \\
& +\left\langle\frac{1}{\tau_{t}} \boldsymbol{n} \times\left(\boldsymbol{\gamma}_{t}-\mathcal{W}_{\boldsymbol{\gamma}_{t}}\right), \boldsymbol{n} \times\left(\boldsymbol{\delta}_{t}-\mathcal{W}_{\boldsymbol{\delta}_{t}}\right)\right\rangle_{\partial \Omega_{h}}, \\
b_{h}\left(\rho, \boldsymbol{\delta}_{t}\right):= & -\left\langle\boldsymbol{U}_{\boldsymbol{\delta}_{t}}+\frac{1}{\tau_{n}} \mathcal{P}_{\boldsymbol{\delta}_{t}}, \rho\right\rangle_{\partial \Omega_{h}}, \\
c_{h}(\phi, \boldsymbol{\delta}):= & \left\langle\boldsymbol{n} \times \operatorname{grad} \phi, \boldsymbol{\delta}_{t}\right\rangle_{\partial \Omega_{h}}, \\
d_{h}(\rho, \psi):= & \left(\mathcal{W}_{\rho}, \mathcal{W}_{\psi}\right)_{\Omega_{h}}+\left\langle\frac{1}{\tau_{n}}\left(\rho-\mathcal{P}_{\rho}\right),\left(\psi-\mathcal{P}_{\psi}\right)\right\rangle_{\partial \Omega_{h}} \\
& +\left\langle\frac{1}{\tau_{t}} \boldsymbol{n} \times \mathcal{W}_{\rho}, \boldsymbol{n} \times \mathcal{W}_{\psi}\right\rangle_{\partial \Omega_{h}}, \\
e_{h}(\phi, \psi):= & -\langle\operatorname{grad} \phi \cdot \boldsymbol{n}, \psi\rangle_{\partial \Omega_{h}},
\end{array}
$$

and

$$
\begin{aligned}
\ell_{1}\left(\boldsymbol{\delta}_{t}\right) & :=-\left(\boldsymbol{f}, \boldsymbol{U}_{\boldsymbol{\delta}_{t}}\right)_{\Omega_{h}}-\left\langle\boldsymbol{g} \times \boldsymbol{n}, \boldsymbol{\delta}_{t}\right\rangle_{\partial \Omega} \\
\ell_{2}(\psi) & :=-\left(\boldsymbol{f}, \mathfrak{u}_{\psi}\right)_{\Omega_{h}}-\langle\boldsymbol{g} \cdot \boldsymbol{n}, \psi\rangle_{\partial \Omega} \\
\ell_{3}(\psi) & :=+(\boldsymbol{f}, \operatorname{grad} \xi)_{\Omega_{h}}
\end{aligned}
$$

3.5. Summary. We have shown how to hybridize the HDG methods in four different ways according to the choice of globally coupled variables. These variables are described in Table 3.1 for each of the hybridizations we considered. They are

\begin{tabular}{|c|c|c|c|c|c|}
\hline type & \multicolumn{3}{|c|}{ unknowns } & \multicolumn{2}{|c|}{ jump conditions } \\
\hline I & $\left(\widehat{\boldsymbol{u}}_{h}\right)_{t}$ & $\widehat{p}_{h}$ & & $\| \boldsymbol{n} \times\left(\widehat{\boldsymbol{\omega}}_{h}\right)_{t} \rrbracket=\mathbf{0}$ & $\llbracket\left(\widehat{\boldsymbol{u}}_{h}\right)_{n} \cdot \boldsymbol{n} \rrbracket=0$ \\
\hline II & $\left(\widehat{\boldsymbol{u}}_{h}\right)_{t}$ & $\left(\widehat{\boldsymbol{u}}_{h}\right)_{n}$ & $\bar{p}_{h}$ & $\llbracket \boldsymbol{n} \times\left(\widehat{\boldsymbol{\omega}}_{h}\right)_{t} \rrbracket=\mathbf{0}$ & $\llbracket \hat{p}_{h} \boldsymbol{n} \rrbracket=\mathbf{0}$ \\
\hline III & $\left(\widehat{\boldsymbol{\omega}}_{h}\right)_{t}$ & $\left(\widehat{\boldsymbol{u}}_{h}\right)_{n}$ & $\bar{p}_{h}$ & $\llbracket\left(\widehat{\boldsymbol{u}}_{h}\right)_{t} \times \boldsymbol{n} \rrbracket=\mathbf{0}$ & $\llbracket \widehat{p}_{h} \boldsymbol{n} \rrbracket=\mathbf{0}$ \\
\hline IV & $\left(\widehat{\boldsymbol{\omega}}_{h}\right)_{t}$ & $\widehat{p}_{h}$ & $\phi_{h}$ & $\llbracket\left(\widehat{\boldsymbol{u}}_{h}\right)_{t} \times \boldsymbol{n} \rrbracket=\mathbf{0}$ & $\llbracket\left(\widehat{\boldsymbol{u}}_{h}\right)_{n} \cdot \boldsymbol{n} \rrbracket=0$ \\
\hline
\end{tabular}
referred to as unknowns therein since all the other variables can be eliminated from the original equations. The corresponding discrete transmission conditions appear alongside under the heading jump conditions. The primary motivation for all these hybridizations is the reduction in the number of global degrees of freedom achieved by the elimination of volumetric unknowns $\boldsymbol{\omega}_{h}, \boldsymbol{u}_{h}$ and $p_{h}$. The variational equations on the mesh faces that we derived in each type result in significantly smaller systems, especially in the high order case.

TABLE 3.1

The unknowns and jump conditions for the hybridizations

For DG methods, the possibility to derive a hybridized formulation is strongly dependent on the structure of the numerical traces. Although we gave expressions for the numerical traces in the traditional DG format as in (2.5), we should note that the numerical traces on which the jump conditions are imposed can be expressed element by element. Indeed, on the boundary of each mesh element $K$, the numerical traces 
on which the jump conditions are imposed, have the following expressions using the values of variables from just that element:

$$
\begin{gathered}
\text { Type I: } \begin{cases}\left(\widehat{\boldsymbol{\omega}}_{h}\right)_{t}=\left(\boldsymbol{\omega}_{h}\right)_{t}+\tau_{t}\left(\boldsymbol{u}_{h}-\left(\widehat{\boldsymbol{u}}_{h}\right)_{t}\right) \times \boldsymbol{n}, & \text { on } \partial K \\
\left(\widehat{\boldsymbol{u}}_{h}\right)_{n}=\left(\boldsymbol{u}_{h}\right)_{n}+\frac{1}{\tau_{n}}\left(p_{h}-\widehat{p}_{h}\right) \boldsymbol{n} & \text { on } \partial K,\end{cases} \\
\text { Type II: }\left\{\begin{array}{cc}
\left(\widehat{\boldsymbol{\omega}}_{h}\right)_{t}=\left(\boldsymbol{\omega}_{h}\right)_{t}+\tau_{t}\left(\boldsymbol{u}_{h}-\left(\widehat{\boldsymbol{u}}_{h}\right)_{t}\right) \times \boldsymbol{n}, & \text { on } \partial K, \\
\widehat{p}_{h}=p_{h}+\tau_{n}\left(\boldsymbol{u}_{h}-\left(\widehat{\boldsymbol{u}}_{h}\right)_{n}\right) \cdot \boldsymbol{n} & \text { on } \partial K,
\end{array}\right. \\
\text { Type III: }\left\{\begin{array}{cc}
\left(\widehat{\boldsymbol{u}}_{h}\right)_{t}=\left(\boldsymbol{u}_{h}\right)_{t}+\frac{1}{\tau_{t}} \boldsymbol{n} \times\left(\boldsymbol{\omega}_{h}-\left(\widehat{\boldsymbol{\omega}}_{h}\right)_{t}\right), & \text { on } \partial K, \\
\widehat{p}_{h}=p_{h}+\tau_{n}\left(\boldsymbol{u}_{h}-\left(\widehat{\boldsymbol{u}}_{h}\right)_{n}\right) \cdot \boldsymbol{n}, & \text { on } \partial K,
\end{array}\right. \\
\text { Type IV: }\left\{\begin{array}{cc}
\left(\widehat{\boldsymbol{u}}_{h}\right)_{t}=\left(\boldsymbol{u}_{h}\right)_{t}+\frac{1}{\tau_{t}} \boldsymbol{n} \times\left(\boldsymbol{\omega}_{h}-\left(\widehat{\boldsymbol{\omega}}_{h}\right)_{t}\right) & \text { on } \partial K, \\
\left(\widehat{\boldsymbol{u}}_{h}\right)_{n}=\left(\boldsymbol{u}_{h}\right)_{n}+\frac{1}{\tau_{n}}\left(p_{h}-\left(\widehat{p}_{h}\right)_{t}\right) \boldsymbol{n} . & \text { on } \partial K .
\end{array}\right.
\end{gathered}
$$

Finally, let us note that in the rewritten expressions of the numerical traces above, it is easy to formally set the parameters $\tau_{t}, \tau_{n}$ to either zero or infinity, which gives rise to numerical methods we can think of as being limiting cases of the HDG methods. In Table 3.2, for each of these limiting cases, we give the associated continuity properties of some the components of the approximate solution as well as the corresponding natural hybridizations.

In particular, if we use the hybridizations of type I or IV and formally set $\tau_{n}=\infty$ in (3.29) or (3.32), we immediately obtain that $\boldsymbol{u}_{h} \in H(d i v, \Omega)$, by the jump condition (3.6c), respectively, jump condition (3.27b), for the type I, respectively, type IV, boundary condition. We also immediately see that the discrete incompressibility condition (2.2c) becomes

$$
\left(\operatorname{div} \boldsymbol{u}_{h}, q\right)_{\Omega_{h}}=0 \quad \forall q \in P_{h},
$$

and if we assume, as in Proposition 2.1, that

$$
\operatorname{div} \boldsymbol{V}(K) \subset P(K) \quad \forall K \in \Omega_{h},
$$

we can conclude that our approximate velocity $\boldsymbol{u}_{h}$ is strongly incompressible. That is, the distributional divergence of the numerical velocity approximation satisfies $\operatorname{div} \boldsymbol{u}_{h}=0$ in all $\Omega$. It is interesting that even though the space $\boldsymbol{V}_{h}$ is a space of completely discontinuous functions, we are able to recover such a velocity approximation. The first DG methods producing strongly incompressible velocities were introduced, in the framework of the Navier-Stokes equations, in [12] and were later more explicitly developed in [13]; see also [21], where this idea is applied to square and cube elements. Another DG method able to provide strongly incompressible velocities is the method introduced in [3]. It uses a velocity space $\boldsymbol{V}_{h}$ of exactly divergence-free velocities and uses a hybridization technique to avoid the almost impossible task of constructing its bases.

Unfortunately, the above-mentioned methods do not fit in our setting. The methods in $[12,13]$ do not use the vorticity as an unknown; instead, they use the gradient of the velocity. The method in [3] almost fits in our setting except for the fact that the numerical traces for the tangential vorticity and the tangential velocity do not 
coincide for any finite values of $\tau_{t}^{ \pm}$. If, on the other hand, we formally set $\tau_{t}^{-}=\infty$ and then take $\tau_{t}^{+}=0$, we do recover the general form of the numerical traces considered in [3]. However, in that case, the numerical trace for the tangential vorticity becomes independent of the tangential velocity. This is certainly not the case for the scheme treated in [3].

TABLE 3.2

The continuity properties induced by the formal limits.

\begin{tabular}{c|cc}
\hline formal limit & continuity property & hybridization type \\
\hline$\tau_{t}=0$ & $\boldsymbol{\omega}_{h} \in H(\operatorname{curl}, \Omega)$ & I, II \\
$\frac{1}{\tau_{t}}=0$ & $\boldsymbol{u}_{h} \in H(\operatorname{curl}, \Omega)$ & III, IV \\
$\tau_{n}=0$ & $p_{h} \in \mathrm{C}^{0}(\Omega)$ & II, III \\
$\frac{1}{\tau_{n}}=0$ & $\boldsymbol{u}_{h} \in H(\operatorname{div}, \Omega)$ & I, IV \\
\hline
\end{tabular}

In Table 3.3, we describe four special limiting cases. Most finite element methods for the Stokes problem use approximate velocities $\boldsymbol{u}_{h}$ in $\boldsymbol{H}^{1}(\Omega)$, see [2]; they thus correspond to the case $\frac{1}{\tau_{t}}=\frac{1}{\tau_{n}}=0$. The method introduced by Nédélec in [17] corresponds to the case $\tau_{t}=\frac{1}{\tau_{n}}=0$; its hybridization was carried out in $[7,8]$.

TABLE 3.3

Four special formal limits of HDG methods.

\begin{tabular}{c|cc}
\hline & $\tau_{t}=0$ & $\frac{1}{\tau_{t}}=0$ \\
\hline \multirow{4}{*}{$\tau_{n}=0$} & $\boldsymbol{\omega}_{h} \in H(\operatorname{curl}, \Omega)$ & $\boldsymbol{u}_{h} \in H(\operatorname{curl}, \Omega)$ \\
$p_{h} \in \mathrm{C}^{0}(\Omega)$ & $p_{h} \in \mathrm{C}^{0}(\Omega)$ \\
& type II hybridization & type III hybridization \\
& & \\
$\tau_{n}=0$ & $\boldsymbol{\omega}_{h} \in H(\operatorname{curl}, \Omega)$ & $\boldsymbol{u}_{h} \in H(\operatorname{curl}, \Omega)$ \\
& $\boldsymbol{u}_{h} \in H(\operatorname{div}, \Omega)$ & $\boldsymbol{u}_{h} \in H(\operatorname{div}, \Omega)$ \\
& type I hybridization & type IV hybridization \\
\hline
\end{tabular}

\section{Proofs of the characterization theorems.}

4.1. Preliminaries. We begin by proving an auxiliary identity that we will use in all our proofs. It is stated in terms of functions $\left(\mathbf{w}_{h}, \mathbf{u}_{h}, \mathrm{p}_{h}\right)$ in $\boldsymbol{W}_{h} \times \boldsymbol{V}_{h} \times P_{h}$ that are assumed to satisfy the equations

$$
\begin{aligned}
\left(\mathbf{w}_{h}, \boldsymbol{\tau}\right)_{\Omega_{h}}-\left(\mathbf{u}_{h}, \operatorname{curl} \boldsymbol{\tau}\right)_{\Omega_{h}}= & -\left\langle\widehat{\mathbf{u}}_{h}, \boldsymbol{n} \times \boldsymbol{\tau}\right\rangle_{\partial \Omega_{h}}, \\
\left(\mathbf{w}_{h}, \operatorname{curl} \boldsymbol{v}\right)_{\Omega_{h}}-\left(\mathbf{p}_{h}, \operatorname{div} \boldsymbol{v}\right)_{\Omega_{h}}= & (\mathbf{f}, \boldsymbol{v}-\mathbf{P} \boldsymbol{v})_{\Omega_{h}}-\left\langle\widehat{\mathbf{p}}_{h},(\boldsymbol{v}-\mathbf{P} \boldsymbol{v}) \cdot \boldsymbol{n}\right\rangle_{\partial \Omega_{h}} \\
& -\left\langle\widehat{\mathbf{w}}_{h},(\boldsymbol{v}-\mathbf{P} \boldsymbol{v}) \times \boldsymbol{n}\right\rangle_{\partial \Omega_{h}} \\
-\left(\mathbf{u}_{h}, \operatorname{grad} q\right)_{\Omega_{h}}= & -\left\langle\widehat{\mathbf{u}}_{h} \cdot \boldsymbol{n}, q-\mathrm{P} q\right\rangle_{\partial \Omega_{h}},
\end{aligned}
$$

for all $(\boldsymbol{\tau}, \boldsymbol{v}, q) \in \boldsymbol{W}_{h} \times \boldsymbol{V}_{h} \times P_{h}$. Here $\mathbf{P}$ is a projection from $P_{h}$, and $\mathbf{P}$ is a projection from $\boldsymbol{V}_{h}$. Their ranges are denoted by $\bar{\psi}_{h}$ and $\boldsymbol{H}_{h}$, respectively. The symbols $\widehat{\mathbf{w}}_{h}, \widehat{\mathbf{u}}_{h}$, and $\widehat{\mathrm{p}}_{h}$, while evocative of numerical traces, are not assumed to be related to the variables $\left(\mathbf{w}_{h}, \mathbf{u}_{h}, \mathrm{p}_{h}\right)$ as in (2.5), nor are they assumed to be single-valued on $\mathscr{E}_{h}$. They simply denote some given functions on $\partial \Omega_{h}$. 
LEMMA 4.1. Let $\left(\mathbf{w}_{h}, \mathbf{u}_{h}, \mathbf{p}_{h}\right)$ be a function satisfying equations (4.1a) and (4.1c), and let $\left(\mathbf{w}_{h}^{\prime}, \mathbf{u}_{h}^{\prime}, \mathrm{p}_{h}^{\prime}\right)$ be a function satisfying (4.1b) with $\mathbf{f}, \widehat{\mathbf{w}}_{h}$, and $\widehat{\mathbf{p}}_{h}$, replaced by $\mathbf{f}^{\prime}$, $\widehat{\mathbf{w}}_{h}^{\prime}$, and $\widehat{\mathbf{p}}_{h}^{\prime}$, respectively. Then

$$
\begin{aligned}
-\left\langle\widehat{\mathbf{u}}_{h}, \boldsymbol{n} \times \widehat{\mathbf{w}}_{h}^{\prime}+\boldsymbol{n} \widehat{\mathrm{p}}_{h}^{\prime}\right\rangle_{\partial \Omega_{h}}= & \left(\mathbf{w}_{h}, \mathbf{w}_{h}^{\prime}\right)_{\Omega_{h}} \\
& -\left\langle\widehat{\mathbf{u}}_{h}-\mathbf{u}_{h}, \boldsymbol{n} \times\left(\widehat{\mathbf{w}}_{h}^{\prime}-\mathbf{w}_{h}^{\prime}\right)+\boldsymbol{n}\left(\widehat{\mathrm{p}}_{h}^{\prime}-\mathrm{p}_{h}^{\prime}\right)\right\rangle_{\partial \Omega_{h}} \\
& -\left(\mathbf{u}_{h}, \mathbf{f}^{\prime}\right)_{\Omega_{h}},
\end{aligned}
$$

whenever $\left(\mathbf{P u}_{h}, \mathbf{P p}_{h}^{\prime}\right)=(\mathbf{0}, 0)$.

Proof. By equation (4.1a) with $\boldsymbol{\tau}:=\mathbf{w}_{h}^{\prime}$, we have that

$$
\left(\mathbf{w}_{h}, \mathbf{w}_{h}^{\prime}\right)_{\Omega_{h}}=\left(\mathbf{u}_{h}, \operatorname{curl} \mathbf{w}_{h}^{\prime}\right)_{\Omega_{h}}-\left\langle\widehat{\mathbf{u}}_{h}, \boldsymbol{n} \times \mathbf{w}_{h}^{\prime}\right\rangle_{\partial \Omega_{h}}
$$

and so, after integration by parts,

$$
\left(\mathbf{w}_{h}, \mathbf{w}_{h}^{\prime}\right)_{\Omega_{h}}=\left(\operatorname{curl} \mathbf{u}_{h}, \mathbf{w}_{h}^{\prime}\right)_{\Omega_{h}}+\left\langle\mathbf{u}_{h}-\widehat{\mathbf{u}}_{h}, \boldsymbol{n} \times \mathbf{w}_{h}^{\prime}\right\rangle_{\partial \Omega_{h}} .
$$

By (4.1b) with $\boldsymbol{v}:=\mathbf{u}_{h}$ and $\mathbf{w}_{h}, \mathbf{u}_{h}, \mathrm{p}_{h}$, and $\mathbf{f}$ replaced by $\mathbf{w}_{h}^{\prime}, \mathbf{u}_{h}^{\prime}, \mathrm{p}_{h}^{\prime}$, and $\mathbf{f}^{\prime}$, respectively, we get

$$
\begin{aligned}
\left(\mathbf{w}_{h}, \mathbf{w}_{h}^{\prime}\right)_{\Omega_{h}}= & -\left\langle\widehat{\mathbf{w}}_{h}^{\prime},\left(\mathbf{u}_{h}-\mathbf{P} \mathbf{u}_{h}\right) \times \boldsymbol{n}\right\rangle_{\partial \Omega_{h}}+\left\langle\mathbf{u}_{h}-\widehat{\mathbf{u}}_{h}, \boldsymbol{n} \times \mathbf{w}_{h}^{\prime}\right\rangle_{\partial \Omega_{h}} \\
& +\left(\mathbf{p}_{h}^{\prime}, \operatorname{div} \mathbf{u}_{h}\right)_{\Omega_{h}}-\left\langle\widehat{\boldsymbol{p}}_{h}^{\prime},\left(\mathbf{u}_{h}-\mathbf{P} \mathbf{u}_{h}\right) \cdot \boldsymbol{n}\right\rangle_{\partial \Omega_{h}}+\left(\mathbf{f}^{\prime}, \mathbf{u}_{h}-\mathbf{P} \mathbf{u}_{h}\right)_{\Omega_{h}}, \\
= & -\left\langle\mathbf{u}_{h}, \boldsymbol{n} \times \widehat{\mathbf{w}}_{h}^{\prime},\right\rangle_{\partial \Omega_{h}}+\left\langle\mathbf{u}_{h}-\widehat{\mathbf{u}}_{h}, \boldsymbol{n} \times \mathbf{w}_{h}^{\prime}\right\rangle_{\partial \Omega_{h}} \\
& +\left(\operatorname{div} \mathbf{u}_{h}, \mathbf{p}_{h}^{\prime}\right)_{\Omega_{h}}-\left\langle\mathbf{u}_{h} \cdot \boldsymbol{n}, \widehat{\mathbf{p}}_{h}^{\prime}\right\rangle_{\partial \Omega_{h}}+\left(\mathbf{u}_{h}, \mathbf{f}^{\prime}\right)_{\Omega_{h}},
\end{aligned}
$$

since $\mathbf{P} \mathbf{u}_{h}=\mathbf{0}$. If we now integrate by parts, we get

$$
\begin{aligned}
\left(\mathbf{w}_{h}, \mathbf{w}_{h}^{\prime}\right)_{\Omega_{h}}= & -\left\langle\mathbf{u}_{h}, \boldsymbol{n} \times \widehat{\mathbf{w}}_{h}^{\prime},\right\rangle_{\partial \Omega_{h}}+\left\langle\mathbf{u}_{h}-\widehat{\mathbf{u}}_{h}, \boldsymbol{n} \times \mathbf{w}_{h}^{\prime}\right\rangle_{\partial \Omega_{h}} \\
& -\left(\mathbf{u}_{h}, \operatorname{grad} \mathbf{p}_{h}^{\prime}\right)_{\Omega_{h}}-\left\langle\mathbf{u}_{h} \cdot \boldsymbol{n}, \widehat{\mathbf{p}}_{h}^{\prime}-\mathbf{p}_{h}^{\prime}\right\rangle_{\partial \Omega_{h}}+\left(\mathbf{u}_{h}, \mathbf{f}^{\prime}\right)_{\Omega_{h}},
\end{aligned}
$$

and by (4.1c) with $q:=\mathrm{p}_{h}^{\prime}$,

$$
\begin{aligned}
\left(\mathbf{w}_{h}, \mathbf{w}_{h}^{\prime}\right)_{\Omega_{h}}= & -\left\langle\mathbf{u}_{h}, \boldsymbol{n} \times \widehat{\mathbf{w}}_{h}^{\prime},\right\rangle_{\partial \Omega_{h}}+\left\langle\mathbf{u}_{h}-\widehat{\mathbf{u}}_{h}, \boldsymbol{n} \times \mathbf{w}_{h}^{\prime}\right\rangle_{\partial \Omega_{h}} \\
& -\left\langle\widehat{\mathbf{u}}_{h} \cdot \boldsymbol{n}, \mathrm{p}_{h}^{\prime}-\mathrm{Pp}_{h}^{\prime}\right\rangle_{\partial \Omega_{h}}-\left\langle\mathbf{u}_{h} \cdot \boldsymbol{n}, \widehat{\mathbf{p}}_{h}^{\prime}-\mathrm{p}_{h}^{\prime}\right\rangle_{\partial \Omega_{h}}+\left(\mathbf{u}_{h}, \mathbf{f}^{\prime}\right)_{\Omega_{h}} \\
= & -\left\langle\widehat{\mathbf{u}}_{h}, \boldsymbol{n} \times \widehat{\mathbf{w}}_{h}^{\prime},\right\rangle_{\partial \Omega_{h}}+\left\langle\mathbf{u}_{h}-\widehat{\mathbf{u}}_{h}, \boldsymbol{n} \times\left(\mathbf{w}_{h}^{\prime}-\widehat{\mathbf{w}}_{h}^{\prime}\right)\right\rangle_{\partial \Omega_{h}} \\
& -\left\langle\widehat{\mathbf{u}}_{h} \cdot \boldsymbol{n}, \widehat{\mathbf{p}}_{h}^{\prime}\right\rangle_{\partial \Omega_{h}}+\left\langle\left(\widehat{\mathbf{u}}_{h}-\mathbf{u}_{h}\right) \cdot \boldsymbol{n}, \widehat{\mathbf{p}}_{h}^{\prime}-\mathrm{p}_{h}^{\prime}\right\rangle_{\partial \Omega_{h}}+\left(\mathbf{u}_{h}, \mathbf{f}^{\prime}\right)_{\Omega_{h}},
\end{aligned}
$$

since $\mathrm{P} p_{h}^{\prime}=0$. The result now follows after a simple rearrangement of terms. This completes the proof.

The following immediate consequence of this result will also be useful.

Corollary 4.2. Let $\left(\mathbf{w}_{h}, \mathbf{u}_{h}, \mathrm{p}_{h}\right)$ be a function satisfying the equations (4.1) and $\left(\mathbf{w}_{h}^{\prime}, \mathbf{u}_{h}^{\prime}, \mathbf{p}_{h}^{\prime}\right)$ a function satisfying equations (4.1) with $\mathbf{f}, \widehat{\mathbf{w}}_{h}, \widehat{\mathbf{u}}_{h}$, and $\widehat{\mathbf{p}}_{h}$, replaced by $\mathbf{f}^{\prime}, \widehat{\mathbf{w}}_{h}^{\prime}, \widehat{\mathbf{u}}_{h}^{\prime}$, and $\widehat{\mathbf{p}}_{h}^{\prime}$, respectively. Then we have

$$
-\left\langle\widehat{\mathbf{u}}_{h}, \boldsymbol{n} \times \widehat{\mathbf{w}}_{h}^{\prime}+\boldsymbol{n} \widehat{\mathrm{p}}_{h}^{\prime}\right\rangle_{\partial \Omega_{h}}+\left(\mathbf{u}_{h}, \mathbf{f}^{\prime}\right)_{\Omega_{h}}=-\left\langle\widehat{\mathbf{u}}_{h}^{\prime}, \boldsymbol{n} \times \widehat{\mathbf{w}}_{h}+\boldsymbol{n} \widehat{\mathrm{p}}_{h}\right\rangle_{\partial \Omega_{h}}+\left(\mathbf{u}_{h}^{\prime}, \mathbf{f}\right)_{\Omega_{h}},
$$

provided $\left(\mathbf{P u}_{h}, \mathrm{Pp}_{h}\right)=\left(\mathbf{P} \mathbf{u}_{h}^{\prime}, \mathrm{Pp}_{h}^{\prime}\right)=(\mathbf{0}, 0)$ and

$-\left\langle\widehat{\mathbf{u}}_{h}-\mathbf{u}_{h}, \boldsymbol{n} \times\left(\widehat{\mathbf{w}}_{h}^{\prime}-\mathbf{w}_{h}^{\prime}\right)+\boldsymbol{n}\left(\widehat{\mathrm{p}}_{h}^{\prime}-\mathrm{p}_{h}^{\prime}\right)\right\rangle_{\partial \Omega_{h}}=-\left\langle\widehat{\mathbf{u}}_{h}^{\prime}-\mathbf{u}_{h}^{\prime}, \boldsymbol{n} \times\left(\widehat{\mathbf{w}}_{h}-\mathbf{w}_{h}\right)+\boldsymbol{n}\left(\widehat{\mathrm{p}}_{h}-\mathrm{p}_{h}\right)\right\rangle_{\partial \Omega_{h}}$. 
4.2. Proof of the characterization of Theorem 3.2. To prove the characterization Theorem 3.2, we are going to use several key identities gathered in the following result. Recall the definitions of specific local solutions in (3.7) (such as $\boldsymbol{\mathcal { W }}_{\boldsymbol{\lambda}_{t}}, \boldsymbol{\mathcal { U }}_{\boldsymbol{\lambda}_{t}}$, etc.). We denote by $\widehat{\mathcal{W}}_{\odot}$ and $\widehat{\mathfrak{U}}_{\odot}$ the corresponding numerical traces, for all choices of the subscript " $\odot$ " that make sense in the discussion of this hybridization case:

$$
\begin{aligned}
\widehat{\mathcal{W}}_{\boldsymbol{\lambda}_{t}} & =\mathcal{W}_{\boldsymbol{\lambda}_{t}}+\tau_{t}\left(\mathcal{U}_{\boldsymbol{\lambda}_{t}}-\boldsymbol{\lambda}_{t}\right) \times \boldsymbol{n}, & \widehat{\mathfrak{U}}_{\boldsymbol{\lambda}_{t}} & =\mathcal{U}_{\boldsymbol{\lambda}_{t}}+\frac{1}{\tau_{n}} \mathcal{P}_{\boldsymbol{\lambda}_{t}} \boldsymbol{n} \\
\widehat{\mathcal{W}}_{\rho} & =\mathcal{W}_{\rho}+\tau_{t}\left(\mathfrak{U}_{\rho} \times \boldsymbol{n}\right), & \widehat{\mathfrak{U}}_{\rho} & =\mathfrak{U}_{\rho}+\frac{1}{\tau_{n}}\left(\mathcal{P}_{\rho}-\rho\right) \boldsymbol{n} \\
\widehat{\mathcal{W}}_{\boldsymbol{f}} & =\mathcal{W}_{\boldsymbol{f}}+\tau_{t}\left(\mathfrak{U}_{\boldsymbol{f}} \times \boldsymbol{n}\right), & \widehat{\mathfrak{U}}_{\boldsymbol{f}} & =\mathfrak{U}_{\boldsymbol{f}}+\frac{1}{\tau_{n}} \mathcal{P}_{\boldsymbol{f}} \boldsymbol{n}
\end{aligned}
$$

Clearly these equations are inherited from the definitions $(3.4 \mathrm{~d})$ and $(3.4 \mathrm{e})$.

Lemma 4.3 (Elementary identities). For any $\boldsymbol{\lambda}_{t}, \boldsymbol{\mu}_{t} \in \boldsymbol{L}^{2}\left(\mathscr{E}_{h}\right)$, any $\rho, \psi \in L^{2}\left(\mathscr{E}_{h}\right)$ and any $\boldsymbol{f} \in \boldsymbol{L}^{2}(\Omega)$, we have

$$
\begin{aligned}
-\left\langle\llbracket \boldsymbol{n} \times \widehat{\mathcal{W}}_{\boldsymbol{\lambda}_{t}} \rrbracket, \boldsymbol{\mu}_{t}\right\rangle_{\mathscr{E}_{h}}= & \left(\mathcal{W}_{\boldsymbol{\lambda}_{t}}, \mathcal{W}_{\boldsymbol{\mu}_{t}}\right)_{\Omega_{h}}+\left\langle\tau_{t}\left(\boldsymbol{\lambda}_{t}-\boldsymbol{U}_{\boldsymbol{\lambda}_{t}}\right)_{t},\left(\boldsymbol{\mu}_{t}-\boldsymbol{U}_{\boldsymbol{\mu}_{t}}\right)_{t}\right\rangle_{\partial \Omega_{h}} \\
& +\left\langle\frac{1}{\tau_{n}} \mathcal{P}_{\boldsymbol{\lambda}_{t}}, \mathcal{P}_{\boldsymbol{\mu}_{t}}\right\rangle_{\partial \Omega_{h}} \\
-\left\langle\llbracket \boldsymbol{n} \times \widehat{\mathcal{W}}_{\rho} \rrbracket, \boldsymbol{\mu}_{t}\right\rangle_{\mathscr{E}_{h}}= & \left\langle\llbracket \widehat{\mathcal{U}}_{\boldsymbol{\mu}_{t}} \cdot \boldsymbol{n} \rrbracket, \rho\right\rangle_{\mathscr{E}_{h}}, \\
-\left\langle\llbracket \boldsymbol{n} \times \widehat{\mathcal{W}}_{\boldsymbol{f}} \rrbracket, \boldsymbol{\mu}_{t}\right\rangle_{\mathscr{E}_{h}}= & -\left(\boldsymbol{f}, \boldsymbol{u}_{\boldsymbol{\mu}_{t}}\right)_{\Omega_{h}} .
\end{aligned}
$$

and

$$
\begin{aligned}
-\left\langle\llbracket \widehat{\mathcal{U}}_{\boldsymbol{\lambda}_{t}} \cdot \boldsymbol{n} \rrbracket, \psi\right\rangle_{\mathscr{E}_{h}}= & \left\langle\llbracket \boldsymbol{n} \times \widehat{\mathcal{W}}_{\psi} \rrbracket, \boldsymbol{\lambda}_{t}\right\rangle_{\mathscr{E}_{h}}, \\
-\left\langle\llbracket \widehat{\boldsymbol{u}}_{\rho} \cdot \boldsymbol{n} \rrbracket, \psi\right\rangle_{\mathscr{E}_{h}}= & \left(\boldsymbol{\mathcal { W }}_{\rho}, \boldsymbol{\mathcal { W }}_{\psi}\right)_{\Omega_{h}}+\left\langle\tau_{t}\left(\boldsymbol{U}_{\rho}\right)_{t},\left(\boldsymbol{U}_{\psi}\right)_{t}\right\rangle_{\partial \Omega_{h}} \\
& +\left\langle\frac{1}{\tau_{n}}\left(\mathcal{P}_{\rho}-\rho\right),\left(\mathcal{P}_{\psi}-\psi\right)\right\rangle_{\partial \Omega_{h}} \\
-\left\langle\llbracket \widehat{\mathfrak{u}}_{\boldsymbol{f}} \cdot \boldsymbol{n} \rrbracket, \psi\right\rangle_{\mathscr{E}_{h}}= & +\left(\boldsymbol{f}, \boldsymbol{u}_{\psi}\right)_{\Omega_{h}} .
\end{aligned}
$$

Proof. In all the applications of Lemma 4.1 and Corollary 4.2 in this proof, we take $(\mathbf{P}, \mathbf{P})=(\mathbf{0}, 0)$. Observe that (4.1) is satisfied by $\left(\mathbf{w}_{h}, \mathbf{u}_{h}, \mathbf{p}_{h}\right)=\left(\mathcal{W}_{\boldsymbol{\mu}_{t}}, \boldsymbol{U}_{\boldsymbol{\mu}_{t}}, \mathcal{P}_{\boldsymbol{\mu}_{t}}\right)$ if we set

$$
\left(\widehat{\mathbf{w}}_{h},\left(\widehat{\mathbf{u}}_{h}\right)_{t},\left(\widehat{\mathbf{u}}_{h}\right)_{n}, \widehat{\mathrm{p}}_{h}, \mathbf{f}\right)=\left(\widehat{\mathcal{W}}_{\boldsymbol{\mu}_{t}}, \boldsymbol{\mu}_{t},\left(\widehat{\boldsymbol{u}}_{\boldsymbol{\mu}_{t}}\right)_{n}, 0, \mathbf{0}\right) .
$$

The system (4.1) is also satisfied by $\left(\mathbf{w}_{h}^{\prime}, \mathbf{u}_{h}^{\prime}, \mathrm{p}_{h}^{\prime}\right)=\left(\boldsymbol{\mathcal { W }}_{\boldsymbol{\lambda}_{t}}, \boldsymbol{U}_{\boldsymbol{\lambda}_{t}}, \mathcal{P}_{\boldsymbol{\lambda}_{t}}\right)$, if we set

$$
\left(\widehat{\mathbf{w}}_{h}^{\prime},\left(\widehat{\mathbf{u}}_{h}^{\prime}\right)_{t},\left(\widehat{\mathbf{u}}_{h}^{\prime}\right)_{n}, \widehat{\mathbf{p}}_{h}^{\prime}, \mathbf{f}^{\prime}\right)=\left(\widehat{\mathcal{W}}_{\boldsymbol{\lambda}_{t}}, \boldsymbol{\lambda}_{t},\left(\widehat{\boldsymbol{u}}_{\boldsymbol{\lambda}_{t}}\right)_{n}, 0, \mathbf{0}\right) .
$$

Hence, by Lemma 4.1,

$$
\begin{aligned}
-\left\langle\llbracket n \times \widehat{\mathcal{W}}_{\boldsymbol{\lambda}_{t}} \rrbracket, \boldsymbol{\mu}_{t}\right\rangle_{\mathscr{E}_{h}}=\left(\mathcal{W}_{\boldsymbol{\lambda}_{t}}, \mathcal{W}_{\boldsymbol{\mu}_{t}}\right)_{\Omega_{h}} & -\left\langle\boldsymbol{\mu}_{t}-\mathcal{U}_{\boldsymbol{\mu}_{t}}, \boldsymbol{n} \times\left(\widehat{\mathcal{W}}_{\boldsymbol{\lambda}_{t}}-\mathcal{W}_{\boldsymbol{\lambda}_{t}}\right)\right\rangle_{\partial \Omega_{h}} \\
& -\left\langle\widehat{\boldsymbol{U}}_{\boldsymbol{\mu}_{t}}-\boldsymbol{U}_{\boldsymbol{\mu}_{t}}, \boldsymbol{n}\left(0-\mathcal{P}_{\boldsymbol{\lambda}_{t}}\right)\right\rangle_{\partial \Omega_{h}} .
\end{aligned}
$$

The first identity of the lemma follows from this and the identities defining the numerical traces such as (4.2). 
The second identity of the lemma follows just as the fourth; see below. The third identity follows from Corollary 4.2. It is easy to check that the conditions of the corollary are satisfied with

$$
\begin{array}{ll}
\left(\mathbf{w}_{h}, \mathbf{u}_{h}, \mathrm{p}_{h}\right)=\left(\mathcal{W}_{\boldsymbol{\mu}_{t}}, \boldsymbol{u}_{\boldsymbol{\mu}_{t},}, \mathcal{P}_{\boldsymbol{\mu}_{t}}\right), & \left(\widehat{\mathbf{w}}_{h},\left(\widehat{\mathbf{u}}_{h}\right)_{t},\left(\widehat{\mathbf{u}}_{h}\right)_{n}, \widehat{p}_{h}, \mathbf{f}\right)=\left(\widehat{\mathcal{W}}_{\boldsymbol{\mu}_{t},}, \boldsymbol{\mu}_{t},\left(\widehat{\boldsymbol{u}}_{\boldsymbol{\mu}_{t}}\right)_{n}, 0, \mathbf{0}\right), \\
\left(\mathbf{w}_{h}^{\prime}, \mathbf{u}_{h}^{\prime}, \mathbf{p}_{h}^{\prime}\right)=\left(\mathcal{W}_{\boldsymbol{f}}, \boldsymbol{u}_{\boldsymbol{f}}, \mathcal{P}_{\boldsymbol{f}}\right), & \left(\widehat{\mathbf{w}}_{h}^{\prime},\left(\widehat{\mathbf{u}}_{h}^{\prime}\right)_{t},\left(\widehat{\mathbf{u}}_{h}^{\prime}\right)_{n}, \widehat{\boldsymbol{p}}_{h}^{\prime}, \mathbf{f}^{\prime}\right)=\left(\widehat{\mathcal{W}}_{\boldsymbol{f}}, \mathbf{0},\left(\widehat{\boldsymbol{u}}_{\boldsymbol{f}}\right)_{n}, 0, \boldsymbol{f}\right) .
\end{array}
$$

Hence the corollary implies that

$$
\begin{aligned}
-\left\langle\boldsymbol{u}_{t}, \boldsymbol{n} \times \widehat{\boldsymbol{W}}_{\boldsymbol{f}}\right\rangle_{\partial \Omega_{h}}+\left(\boldsymbol{u}_{\boldsymbol{\mu}}, \boldsymbol{f}\right)_{\Omega_{h}} & =-\left\langle\widehat{\mathbf{u}}_{h}, \boldsymbol{n} \times \widehat{\mathbf{w}}_{h}^{\prime}\right\rangle_{\partial \Omega_{h}}+\left(\mathbf{u}_{h}, \mathbf{f}^{\prime}\right)_{\Omega_{h}} \\
& =-\left\langle\widehat{\mathbf{u}}_{h}^{\prime}, \boldsymbol{n} \times \widehat{\mathbf{w}}_{h}\right\rangle_{\partial \Omega_{h}}+\left(\mathbf{u}_{h}^{\prime}, \mathbf{f}\right)_{\Omega_{h}} \\
& =-\left\langle\mathbf{0}, \boldsymbol{n} \times \widehat{\boldsymbol{W}}_{\boldsymbol{\mu}}\right\rangle_{\partial \Omega_{h}} \\
& =0
\end{aligned}
$$

and the required identity follows.

The fourth identity also follows from Corollary 4.2 after verifying its conditions with

$$
\begin{array}{ll}
\left(\mathbf{w}_{h}, \mathbf{u}_{h}, \mathrm{p}_{h}\right)=\left(\mathcal{W}_{\boldsymbol{\lambda}_{t}}, \boldsymbol{u}_{\boldsymbol{\lambda}_{t}}, \mathcal{P}_{\boldsymbol{\lambda}_{t}}\right), & \left(\widehat{\mathbf{w}}_{h},\left(\widehat{\mathbf{u}}_{h}\right)_{t},\left(\widehat{\mathbf{u}}_{h}\right)_{n}, \widehat{\mathrm{p}}_{h}, \mathbf{f}\right)=\left(\widehat{\mathcal{W}}_{\boldsymbol{\lambda}_{t}}, \boldsymbol{\lambda}_{t},\left(\widehat{\mathbf{u}}_{\boldsymbol{\lambda}_{t}}\right)_{n}, 0, \mathbf{0}\right), \\
\left(\mathbf{w}_{h}^{\prime}, \mathbf{u}_{h}^{\prime}, \mathrm{p}_{h}^{\prime}\right)=\left(\boldsymbol{\mathcal { W }}_{\psi}, \boldsymbol{u}_{\psi}, \mathcal{P}_{\psi}\right), & \left(\widehat{\mathbf{w}}_{h}^{\prime},\left(\widehat{\mathbf{u}}_{h}^{\prime}\right)_{t},\left(\widehat{\mathbf{u}}_{h}^{\prime}\right)_{n}, \widehat{\mathrm{p}}_{h}^{\prime}, \mathbf{f}^{\prime}\right)=\left(\widehat{\mathcal{W}}_{\psi}, \mathbf{0},\left(\widehat{\boldsymbol{u}}_{\psi}\right)_{n}, \psi, \mathbf{0}\right) .
\end{array}
$$

The fifth identity follows from Lemma 4.1 with

$$
\begin{array}{ll}
\left(\mathbf{w}_{h}, \mathbf{u}_{h}, \mathrm{p}_{h}\right)=\left(\mathcal{W}_{\rho}, \boldsymbol{U}_{\rho}, \mathcal{P}_{\rho}\right), & \left(\widehat{\mathbf{w}}_{h},\left(\widehat{\mathbf{u}}_{h}\right)_{t},\left(\widehat{\mathbf{u}}_{h}\right)_{n}, \widehat{\mathbf{p}}_{h}, \mathbf{f}\right)=\left(\widehat{\mathcal{W}}_{\rho}, \mathbf{0},\left(\boldsymbol{U}_{\rho}\right)_{n}, \rho, \mathbf{0}\right) . \\
\left(\mathbf{w}_{h}^{\prime}, \mathbf{u}_{h}^{\prime}, \mathbf{p}_{h}^{\prime}\right)=\left(\mathcal{W}_{\psi}, \boldsymbol{U}_{\psi}, \mathcal{P}_{\psi}\right), & \left(\widehat{\mathbf{w}}_{h}^{\prime},\left(\widehat{\mathbf{u}}_{h}^{\prime}\right)_{t},\left(\widehat{\mathbf{u}}_{h}^{\prime}\right)_{n}, \widehat{\mathbf{p}}_{h}^{\prime}, \mathbf{f}^{\prime}\right)=\left(\widehat{\mathcal{W}}_{\psi}, \mathbf{0},\left(\widehat{\boldsymbol{U}}_{\psi}\right)_{n}, \psi, \mathbf{0}\right) .
\end{array}
$$

The sixth identity follows from Corollary 4.2 after verifying its conditions with

$$
\begin{array}{ll}
\left(\mathbf{w}_{h}, \mathbf{u}_{h}, \mathrm{p}_{h}\right)=\left(\mathcal{W}_{\boldsymbol{f}}, \boldsymbol{u}_{\boldsymbol{f}}, \mathcal{P}_{\boldsymbol{f}}\right), & \left(\widehat{\mathbf{w}}_{h},\left(\widehat{\mathbf{u}}_{h}\right)_{t},\left(\widehat{\mathbf{u}}_{h}\right)_{n}, \widehat{\mathrm{p}}_{h}, \mathbf{f}\right)=\left(\widehat{\mathcal{W}}_{\boldsymbol{\mu}_{t}}, \mathbf{0},\left(\widehat{\mathbf{u}}_{\boldsymbol{f}}\right)_{n}, 0, \boldsymbol{f}\right), \\
\left(\mathbf{w}_{h}^{\prime}, \mathbf{u}_{h}^{\prime}, \mathbf{p}_{h}^{\prime}\right)=\left(\boldsymbol{\mathcal { W }}_{\psi}, \boldsymbol{u}_{\psi}, \mathcal{P}_{\psi}\right), & \left(\widehat{\mathbf{w}}_{h}^{\prime},\left(\widehat{\mathbf{u}}_{h}^{\prime}\right)_{t},\left(\widehat{\mathbf{u}}_{h}^{\prime}\right)_{n}, \widehat{\mathbf{p}}_{h}^{\prime}, \mathbf{f}^{\prime}\right)=\left(\widehat{\mathcal{W}}_{\psi}, \mathbf{0},\left(\widehat{\boldsymbol{u}}_{\psi}\right)_{n}, \psi, \mathbf{0}\right) .
\end{array}
$$

This completes the proof of the lemma.

Proof of Theorem 3.2. By the jump conditions (3.6b) and (3.6c),

$$
\begin{aligned}
-\left\langle\llbracket \boldsymbol{n} \times\left(\widehat{\mathcal{W}}_{\boldsymbol{\lambda}_{t}^{o}}+\widehat{\mathcal{W}}_{\rho}\right) \rrbracket, \boldsymbol{\mu}_{t}\right\rangle_{\mathscr{E}_{h}} & =\left\langle\llbracket \boldsymbol{n} \times\left(\widehat{\mathcal{W}}_{\boldsymbol{f}}+\widehat{\mathcal{W}}_{\boldsymbol{g}}\right) \rrbracket, \boldsymbol{\mu}_{t}\right\rangle_{\mathscr{E}_{h}}, \\
-\left\langle\llbracket\left(\widehat{\mathcal{U}}_{\boldsymbol{\lambda}_{t}^{o}}+\widehat{\mathfrak{U}}_{\rho}\right) \cdot \boldsymbol{n} \rrbracket, \psi\right\rangle_{\mathscr{E}_{h}} & =\left\langle\llbracket\left(\widehat{\mathcal{U}}_{\boldsymbol{f}}+\widehat{\mathfrak{U}}_{\boldsymbol{g}}\right) \cdot \boldsymbol{n} \rrbracket, \psi\right\rangle_{\mathscr{E}_{h}}-\langle\boldsymbol{g} \cdot \boldsymbol{n}, \psi\rangle_{\partial \Omega} .
\end{aligned}
$$

By Lemma 4.3, we have that

$$
\begin{aligned}
& -\left\langle\llbracket \boldsymbol{n} \times \widehat{\mathcal{W}}_{\boldsymbol{\lambda}_{t}^{o}} \rrbracket, \boldsymbol{\mu}_{t}\right\rangle_{\mathscr{E}_{h}}=a_{h}\left(\boldsymbol{\lambda}_{t}^{o}, \boldsymbol{\mu}_{t}\right), \quad-\left\langle\llbracket \widehat{\mathcal{U}}_{\boldsymbol{\lambda}_{t}^{o}} \cdot \boldsymbol{n} \rrbracket, \psi\right\rangle_{\mathscr{E}_{h}}=-b_{h}\left(\psi, \boldsymbol{\lambda}_{t}^{o}\right), \\
& -\left\langle\llbracket \boldsymbol{n} \times \widehat{\mathcal{W}}_{\rho} \rrbracket, \boldsymbol{\mu}_{t}\right\rangle_{\mathscr{E}_{h}}=b_{h}\left(\rho, \boldsymbol{\mu}_{t}\right), \quad-\left\langle\llbracket \widehat{\boldsymbol{U}}_{\rho} \cdot \boldsymbol{n} \rrbracket, \psi\right\rangle_{\mathscr{E}_{h}}=c_{h}(\rho, \psi) .
\end{aligned}
$$

In order to prove (3.8a) and (3.8b), we now only have to show that $\ell_{1}=\tilde{\ell}_{1}$ and $\ell_{2}=\tilde{\ell}_{2}$ where

$$
\begin{aligned}
\tilde{\ell}_{1}\left(\boldsymbol{\mu}_{t}\right) & :=\left\langle\llbracket \boldsymbol{n} \times \widehat{\mathcal{W}}_{\boldsymbol{f}} \rrbracket, \boldsymbol{\mu}_{t}\right\rangle_{\mathscr{E}_{h}}+\left\langle\llbracket \boldsymbol{n} \times \widehat{\mathcal{W}}_{\boldsymbol{g}} \rrbracket, \boldsymbol{\mu}_{t}\right\rangle_{\mathscr{E}_{h}}, \\
\tilde{\ell}_{2}(\psi) & :=\left\langle\llbracket \widehat{\mathcal{U}}_{\boldsymbol{f}} \cdot \boldsymbol{n} \rrbracket, \psi\right\rangle_{\mathscr{E}_{h}}+\left\langle\llbracket \widehat{\mathcal{U}}_{\boldsymbol{g}} \cdot \boldsymbol{n} \rrbracket, \psi\right\rangle_{\mathscr{E}_{h}}-\langle\boldsymbol{g} \cdot \boldsymbol{n}, \psi\rangle_{\partial \Omega}
\end{aligned}
$$


But, again by Lemma 4.3, we have

$$
\begin{aligned}
\tilde{\ell}_{1}\left(\boldsymbol{\mu}_{t}\right) & =\left(\boldsymbol{f}, \boldsymbol{U}_{\boldsymbol{\mu}_{t}}\right)_{\Omega_{h}}-a_{h}\left(\boldsymbol{g}, \boldsymbol{\mu}_{t}\right) \\
& =\ell_{1}\left(\boldsymbol{\mu}_{t}\right) .
\end{aligned}
$$

Similarly, applying Lemma 4.3 one more time,

$$
\begin{aligned}
\widetilde{\ell}_{2}(\psi) & =-\left(\boldsymbol{f}, \mathfrak{u}_{\psi}\right)_{\Omega_{h}}-\left\langle\boldsymbol{g}, \llbracket \boldsymbol{n} \times \widehat{\mathcal{W}}_{\psi} \rrbracket\right\rangle_{\mathscr{E}_{h}}-\langle\boldsymbol{g} \cdot \boldsymbol{n}, \psi\rangle_{\partial \Omega} \\
& =-\left(\boldsymbol{f}, \mathfrak{u}_{\psi}\right)_{\Omega_{h}}+b_{h}\left(\psi, \boldsymbol{g}_{t}\right)-\langle\boldsymbol{g} \cdot \boldsymbol{n}, \psi\rangle_{\partial \Omega} \\
& =\ell_{2}(\psi) .
\end{aligned}
$$

It now only remains to prove that $\left(\boldsymbol{\lambda}_{t}^{o}, \rho\right)$ is the only solution of $(3.8 \mathrm{a})-(3.8 \mathrm{c})$. First observe that the above arguments in fact show that the jump conditions (3.6b) and (3.6c) hold if and only if (3.8a) and (3.8b) hold, respectively. Hence if $\left(\tilde{\boldsymbol{\lambda}}_{t}^{o}, \tilde{\rho}\right)$ is another solution of $(3.8 \mathrm{a})-(3.8 \mathrm{c})$, then the numerical traces generated by $\mathcal{L}^{\mathrm{I}}\left(\tilde{\boldsymbol{\lambda}}_{t}^{o}, \tilde{\rho}, \boldsymbol{f}\right)$ will also satisfy (3.6b) and (3.6c). But then, since (3.8c) implies (3.6d), we find that all the conditions of Theorem 3.1 are verified, so we conclude that $\tilde{\boldsymbol{\lambda}}_{t}^{o}+\boldsymbol{g}_{t}=\left(\widehat{\boldsymbol{u}}_{h}\right)_{t}$ and $\tilde{\rho}=\widehat{p}_{h}$. Since we also have $\left(\boldsymbol{\lambda}_{t}^{o}+\boldsymbol{g}_{t}, \rho\right)=\left(\left(\widehat{\boldsymbol{u}}_{h}\right)_{t}, \widehat{p}_{h}\right)$, we conclude that $\left(\tilde{\boldsymbol{\lambda}}_{t}^{o}, \tilde{\rho}\right)=$ $\left(\boldsymbol{\lambda}_{t}^{o}, \rho\right)$. This completes the proof of Theorem 3.2.

4.3. Proof of the characterization of Theorem 3.4. To prove Theorem 3.4, we proceed as in the previous case and gather several key identities in the following result. Recall the definitions of specific local solutions in (3.16) (such as $\boldsymbol{W}_{\boldsymbol{\lambda}}, \boldsymbol{U}_{\boldsymbol{\lambda}}$, etc.). The numerical traces $\widehat{\mathcal{W}}_{\odot}$ and $\widehat{\mathcal{P}}_{\odot}$ are given by (3.11) for the choices of subscript $\odot$ that make sense here, such as when $\odot$ is $\boldsymbol{\lambda}$, $\bar{\rho}$ or $\boldsymbol{f}$, e.g.,

$$
\widehat{\mathcal{P}}_{\boldsymbol{\lambda}}=\mathcal{P}_{\boldsymbol{\lambda}}+\tau_{n}\left(\mathcal{U}_{\boldsymbol{\lambda}}-\boldsymbol{\lambda}\right) \cdot \boldsymbol{n}, \quad \widehat{\mathcal{W}}_{\bar{\rho}}=\mathcal{W}_{\bar{\rho}}+\tau_{t} \boldsymbol{U}_{\bar{\rho}} \times \boldsymbol{n},
$$

just as in the previous case.

Lemma 4.4 (Elementary identities). For any $\boldsymbol{\lambda}, \boldsymbol{\mu} \in \boldsymbol{L}^{2}\left(\mathscr{E}_{h}\right)$, any $\bar{\rho} \in \ell^{2}\left(\partial \Omega_{h}\right)$ and any $\boldsymbol{f} \in \boldsymbol{L}^{2}(\Omega)$, we have

$$
\begin{aligned}
-\left\langle\llbracket \boldsymbol{n} \times \widehat{\mathcal{W}}_{\boldsymbol{\lambda}}+\widehat{\mathcal{P}}_{\boldsymbol{\lambda}} \boldsymbol{n} \rrbracket, \boldsymbol{\mu}\right\rangle_{\mathscr{E}_{h}} & =\left(\mathcal{W}_{\boldsymbol{\lambda}}, \mathcal{W}_{\boldsymbol{\mu}}\right)_{\Omega_{h}} \\
& +\left\langle\tau_{t}\left(\boldsymbol{\lambda}-\mathcal{U}_{\boldsymbol{\lambda}}\right)_{t},\left(\boldsymbol{\mu}-\boldsymbol{U}_{\boldsymbol{\mu}}\right)_{t}\right\rangle_{\partial \Omega_{h}} \\
& +\left\langle\tau_{n}\left(\boldsymbol{\lambda}-\mathfrak{U}_{\boldsymbol{\lambda}}\right)_{n},\left(\boldsymbol{\mu}-\mathfrak{U}_{\boldsymbol{\mu}}\right)_{n}\right\rangle_{\partial \Omega_{h}}, \\
-\left\langle\llbracket \boldsymbol{n} \times \widehat{\mathcal{W}}_{\bar{\rho}}+\widehat{\mathcal{P}}_{\bar{\rho}} \boldsymbol{n} \rrbracket, \boldsymbol{\mu}\right\rangle_{\mathscr{E}_{h}} & =-\langle\bar{\rho}, \boldsymbol{\mu} \cdot \boldsymbol{n}\rangle_{\partial \Omega_{h}}, \\
-\left\langle\llbracket \boldsymbol{n} \times \widehat{\mathcal{W}}_{\boldsymbol{f}}+\widehat{\mathcal{P}}_{\boldsymbol{f}} \boldsymbol{n} \rrbracket, \boldsymbol{\mu}\right\rangle_{\mathscr{E}_{h}} & =-\left(\boldsymbol{f}, \boldsymbol{U}_{\boldsymbol{\mu}}\right)_{\Omega_{h}} .
\end{aligned}
$$

Proof. The second identity immediately follows because by (3.17),

$$
\boldsymbol{n} \times \widehat{\mathcal{W}}_{\bar{\rho}}+\boldsymbol{n} \widehat{\mathcal{P}}_{\bar{\rho}}=+\bar{\rho} \boldsymbol{n} .
$$

To prove the remaining identities, we set $\mathbf{P}=\mathbf{0}$ and $\mathbf{P} \psi=\bar{\psi}$ (where $\bar{\psi}$ is as defined in (3.12) and apply Lemma 4.1 and Corollary 4.2 appropriately. Indeed, to prove the first identity, first observe that (4.1) is satisfied by

$$
\begin{array}{rlrl}
\left(\mathbf{w}_{h}^{\prime}, \mathbf{u}_{h}^{\prime}, \mathrm{p}_{h}^{\prime}\right) & =\left(\mathcal{W}_{\boldsymbol{\lambda}}, \mathcal{U}_{\boldsymbol{\lambda}}, \mathcal{P}_{\boldsymbol{\lambda}}\right) \quad \text { with } \quad\left(\widehat{\mathbf{w}}_{h}^{\prime}, \widehat{\mathbf{u}}_{h}^{\prime}, \widehat{\mathrm{p}}_{h}^{\prime}, \mathbf{f}^{\prime}\right)=\left(\widehat{\mathcal{W}}_{\boldsymbol{\lambda}}, \boldsymbol{\lambda}, \widehat{\mathcal{P}}_{\boldsymbol{\lambda}}, \mathbf{0}\right), \quad \text { and } \\
\left(\mathbf{w}_{h}, \mathbf{u}_{h}, \mathrm{p}_{h}\right)=\left(\mathcal{W}_{\boldsymbol{\mu}}, \mathcal{U}_{\boldsymbol{\mu}}, \mathcal{P}_{\boldsymbol{\mu}}\right) \quad \text { with } \quad\left(\widehat{\mathbf{w}}_{h}, \widehat{\mathbf{u}}_{h}, \widehat{\mathrm{p}}_{h}, \mathbf{f}\right)=\left(\widehat{\mathcal{W}}_{\boldsymbol{\mu}}, \boldsymbol{\mu}, \widehat{\mathcal{P}}_{\boldsymbol{\mu}}, \mathbf{0}\right) .
\end{array}
$$


Furthermore, $\mathrm{PP}_{\boldsymbol{\lambda}}=0$. Hence the first identity follows by applying Lemma 4.1.

Similarly, the last identity follows from Corollary 4.2 setting

$$
\begin{array}{ll}
\left(\mathbf{w}_{h}^{\prime}, \mathbf{u}_{h}^{\prime}, \mathrm{p}_{h}^{\prime}\right)=\left(\mathcal{W}_{\boldsymbol{f}}, \boldsymbol{u}_{\boldsymbol{f}}, \mathcal{P}_{\boldsymbol{f}}\right) & \left(\widehat{\mathbf{w}}_{h}^{\prime}, \widehat{\mathbf{u}}_{h}^{\prime}, \widehat{\mathrm{p}}_{h}^{\prime}, \mathbf{f}^{\prime}\right)=\left(\widehat{\mathcal{W}}_{\boldsymbol{f}}, \mathbf{0}, \widehat{\mathcal{P}}_{\boldsymbol{f}}, \boldsymbol{f}\right), \quad \text { and } \\
\left(\mathbf{w}_{h}, \mathbf{u}_{h}, \mathrm{p}_{h}\right)=\left(\boldsymbol{\mathcal { W }}_{\boldsymbol{\mu}}, \boldsymbol{u}_{\boldsymbol{\mu}}, \mathcal{P}_{\boldsymbol{\mu}}\right) & \left(\widehat{\mathbf{w}}_{h}, \widehat{\mathbf{u}}_{h}, \widehat{\mathrm{p}}_{h}, \mathbf{f}\right)=\left(\widehat{\mathcal{W}}_{\boldsymbol{\mu}}, \boldsymbol{\mu}, \widehat{\mathcal{P}}_{\boldsymbol{\mu}}, \mathbf{0}\right) .
\end{array}
$$

This completes the proof of the identities.

Proof of Theorem 3.4. By the jump conditions (3.15b) and (3.15c),

$$
\begin{aligned}
-\left\langle\llbracket n \times \widehat{\mathcal{W}}_{\boldsymbol{\lambda}^{\circ}}+\widehat{\mathcal{P}}_{\boldsymbol{\lambda}^{\circ}} \boldsymbol{n}+\boldsymbol{n} \times \widehat{\mathcal{W}}_{\bar{\rho}}+\widehat{\mathcal{P}}_{\bar{\rho}} \boldsymbol{n} \rrbracket, \boldsymbol{\mu}\right\rangle_{\mathscr{E}_{h}} \\
=\left\langle\llbracket \boldsymbol{n} \times \widehat{\mathcal{W}}_{\boldsymbol{f}}+\widehat{\mathcal{P}}_{\boldsymbol{f}} \boldsymbol{n}+\boldsymbol{n} \times \widehat{\mathcal{W}}_{\boldsymbol{g}}+\widehat{\mathcal{P}}_{\boldsymbol{g}} \boldsymbol{n} \rrbracket, \boldsymbol{\mu}\right\rangle_{\mathscr{E}_{h}} .
\end{aligned}
$$

By Lemma 4.4, we have that

$$
\begin{aligned}
-\left\langle\llbracket \boldsymbol{n} \times \widehat{\mathcal{W}}_{\boldsymbol{\lambda}^{o}}+\widehat{\mathcal{P}}_{\boldsymbol{\lambda}^{o}} \boldsymbol{n} \rrbracket, \boldsymbol{\mu}\right\rangle_{\mathscr{E}_{h}} & =a_{h}\left(\boldsymbol{\lambda}^{o}, \boldsymbol{\mu}\right), \\
-\left\langle\llbracket \boldsymbol{n} \times \widehat{\mathcal{W}}_{\bar{\rho}}+\widehat{\mathcal{P}}_{\bar{\rho}} \boldsymbol{n} \rrbracket, \boldsymbol{\mu}\right\rangle_{\mathscr{E}_{h}} & =b_{h}(\bar{\rho}, \boldsymbol{\mu}) .
\end{aligned}
$$

It remains to show that the form $\ell(\cdot)$ of the theorem coincides with $\widetilde{\ell}$ defined by

$$
\tilde{\ell}(\boldsymbol{\mu}):=\left\langle\llbracket \boldsymbol{n} \times \widehat{\mathcal{W}}_{\boldsymbol{f}}+\widehat{\mathcal{P}}_{\boldsymbol{f}} \boldsymbol{n} \rrbracket, \boldsymbol{\mu}\right\rangle_{\mathscr{E}_{h}}+\left\langle\llbracket \boldsymbol{n} \times \widehat{\mathcal{W}}_{\boldsymbol{g}}+\widehat{\mathcal{P}}_{\boldsymbol{g}} \boldsymbol{n} \rrbracket, \boldsymbol{\mu}\right\rangle_{\mathscr{E}_{h}}
$$

But, again by Lemma 4.4, we have

$$
\begin{aligned}
\widetilde{\ell}(\boldsymbol{\mu}) & =\left(\boldsymbol{f}, \boldsymbol{U}_{\boldsymbol{\mu}}\right)_{\Omega_{h}}-a_{h}(\boldsymbol{g}, \boldsymbol{\mu}) \\
& =\ell(\boldsymbol{\mu}) .
\end{aligned}
$$

The proof of uniqueness of the trace solution $\left(\boldsymbol{\lambda}^{o}, \bar{\rho}\right)$ proceeds as in the type I case, so we omit it.

4.4. Proof of the characterization of Theorem 3.6. We now prove Theorem 3.6, using the identities gathered in the next lemma. The notation for the numerical traces of the form $\widehat{\boldsymbol{U}}_{\odot}$ and $\widehat{\mathcal{P}}_{\odot}$ have meanings inherited from $(3.20 \mathrm{e})$ and $(3.20 \mathrm{f})$ as in the previous cases.

Lemma 4.5 (Elementary identities). For any $\boldsymbol{\gamma}_{t}, \boldsymbol{\delta}_{t} \in \boldsymbol{L}^{2}\left(\mathscr{E}_{h}\right)$, any $\boldsymbol{\lambda}_{n}, \boldsymbol{\mu}_{n} \in$ $\boldsymbol{L}^{2}\left(\mathscr{E}_{h}\right)$, any $\rho, \psi \in L^{2}\left(\mathscr{E}_{h}\right)$ and any $\boldsymbol{f} \in \boldsymbol{L}^{2}(\Omega)$, we have

$$
\begin{aligned}
-\left\langle\llbracket \widehat{\mathcal{U}}_{\boldsymbol{\gamma}_{t}} \times \boldsymbol{n} \rrbracket, \boldsymbol{\delta}_{t}\right\rangle_{\mathscr{E}_{h}} & =\left(\boldsymbol{W}_{\boldsymbol{\gamma}_{t}}, \boldsymbol{W}_{\boldsymbol{\delta}_{t}}\right)_{\Omega_{h}}+\left\langle\tau_{n}\left(\boldsymbol{U}_{\boldsymbol{\gamma}_{t}}\right)_{n},\left(\boldsymbol{U}_{\boldsymbol{\delta}_{t}}\right)_{n}\right\rangle_{\partial \Omega_{h}} \\
& +\left\langle\frac{1}{\tau_{t}} \boldsymbol{n} \times\left(\boldsymbol{\gamma}_{t}-\mathcal{W}_{\boldsymbol{\gamma}_{t}}\right), \boldsymbol{n} \times\left(\boldsymbol{\delta}_{t}-\boldsymbol{W}_{\boldsymbol{\delta}_{t}}\right)\right\rangle_{\partial \Omega_{h}}, \\
-\left\langle\llbracket \widehat{\mathcal{U}}_{\boldsymbol{\lambda}_{n}} \times \boldsymbol{n} \rrbracket, \boldsymbol{\delta}_{t}\right\rangle_{\mathscr{E}_{h}} & =\left\langle\llbracket \widehat{\mathcal{P}}_{\boldsymbol{\delta}_{t}} \boldsymbol{n} \rrbracket, \boldsymbol{\lambda}_{n}\right\rangle_{\mathscr{E}_{h}}, \\
-\left\langle\llbracket \widehat{\mathcal{U}}_{\bar{\rho}} \times \boldsymbol{n} \rrbracket, \boldsymbol{\delta}_{t}\right\rangle_{\mathscr{E}_{h}} & =0, \\
-\left\langle\llbracket \widehat{\mathcal{U}}_{\boldsymbol{f}} \times \boldsymbol{n} \rrbracket, \boldsymbol{\delta}_{t}\right\rangle_{\mathscr{E}_{h}} & =\left(\boldsymbol{f}, \boldsymbol{u}_{\boldsymbol{\delta}_{t}}\right)_{\Omega_{h}},
\end{aligned}
$$

and

$$
\begin{aligned}
-\left\langle\llbracket \widehat{\mathcal{P}}_{\boldsymbol{\gamma}_{t}} \boldsymbol{n} \rrbracket, \boldsymbol{\mu}_{n}\right\rangle_{\mathscr{E}_{h}} & =\left\langle\llbracket \widehat{\mathcal{U}}_{\boldsymbol{\mu}_{n}} \times \boldsymbol{n} \rrbracket, \boldsymbol{\gamma}_{t}\right\rangle_{\mathscr{E}_{h}}, \\
-\left\langle\llbracket \widehat{\mathcal{P}}_{\boldsymbol{\lambda}_{n}} \boldsymbol{n} \rrbracket, \boldsymbol{\mu}_{n}\right\rangle_{\mathscr{E}_{h}} & =\left(\boldsymbol{\mathcal { W }}_{\boldsymbol{\lambda}_{n}}, \boldsymbol{W}_{\boldsymbol{\mu}_{n}}\right)_{\Omega_{h}}+\left\langle\frac{1}{\tau_{t}} \boldsymbol{n} \times \mathcal{W}_{\boldsymbol{\lambda}_{n}}, \boldsymbol{n} \times \mathcal{W}_{\boldsymbol{\mu}_{n}}\right\rangle_{\partial \Omega_{h}} \\
& +\left\langle\tau_{n}\left(\boldsymbol{\lambda}_{n}-\boldsymbol{U}_{\boldsymbol{\lambda}_{n}}\right)_{n},\left(\boldsymbol{\mu}_{n}-\boldsymbol{U}_{\boldsymbol{\mu}_{n}}\right)_{n}\right\rangle_{\partial \Omega_{h}} \\
-\left\langle\llbracket \widehat{\mathcal{P}}_{\bar{\rho}} \boldsymbol{n} \rrbracket, \boldsymbol{\mu}_{n}\right\rangle_{\mathscr{E}_{h}} & =-\left\langle\bar{\rho}, \boldsymbol{\mu}_{n} \cdot \boldsymbol{n}\right\rangle_{\partial \Omega_{h}} \\
-\left\langle\llbracket \widehat{\mathcal{P}}_{\boldsymbol{f}} \boldsymbol{n} \rrbracket, \boldsymbol{\mu}_{n}\right\rangle_{\mathscr{E}_{h}} & =-\left(\boldsymbol{f}, \boldsymbol{U}_{\boldsymbol{\mu}_{n}}\right)_{\Omega_{h}} .
\end{aligned}
$$


Proof. The third and seventh identities immediately follow because $\widehat{\mathfrak{U}}_{\bar{\rho}}=\mathbf{0}$ and $\widehat{\mathcal{P}}_{\bar{\rho}}=\bar{\rho}$.

For proving the remaining identities, we apply Lemma 4.1 and Corollary 4.2 with $\mathbf{P}=\mathbf{0}$ and $\mathbf{P} \psi=\bar{\psi}$. To prove the first identity, observe that (4.1) is satisfied by

$$
\left(\mathbf{w}_{h}^{\prime}, \mathbf{u}_{h}^{\prime}, \mathbf{p}_{h}^{\prime}\right)=\left(\mathcal{W}_{\boldsymbol{\delta}_{t}}, \boldsymbol{U}_{\boldsymbol{\delta}_{t}}, \mathcal{P}_{\boldsymbol{\delta}_{t}}\right) \text { with }\left(\widehat{\mathbf{w}}_{h}^{\prime},\left(\widehat{\mathbf{u}}_{h}^{\prime}\right)_{t},\left(\widehat{\mathbf{u}}_{h}^{\prime}\right)_{n}, \widehat{\mathbf{p}}_{h}^{\prime}, \mathbf{f}^{\prime}\right)=\left(\boldsymbol{\delta}_{t},\left(\widehat{\boldsymbol{U}}_{\delta_{t}}\right)_{t}, 0, \widehat{\mathcal{P}}_{\boldsymbol{\delta}_{t}}, \mathbf{0}\right) \text {. }
$$

Eq. (4.1) is also satisfied by

$$
\left(\mathbf{w}_{h}^{\prime}, \mathbf{u}_{h}^{\prime}, \mathrm{p}_{h}^{\prime}\right)=\left(\mathcal{W}_{\gamma_{t}}, \boldsymbol{u}_{\gamma_{t}}, \mathcal{P}_{\gamma_{t}}\right) \text { with }\left(\widehat{\mathbf{w}}_{h}^{\prime},\left(\widehat{\mathbf{u}}_{h}^{\prime}\right)_{t},\left(\widehat{\mathbf{u}}_{h}^{\prime}\right)_{n}, \widehat{\mathbf{p}}_{h}^{\prime}, \mathbf{f}^{\prime}\right)=\left(\boldsymbol{\gamma}_{t},\left(\widehat{\boldsymbol{u}}_{\gamma_{t}}\right)_{t}, 0, \widehat{\mathcal{P}}_{\gamma_{t}}, \mathbf{0}\right) \text {. }
$$

Since we also have $\mathrm{PP}_{\boldsymbol{\delta}_{t}}=0$ because of (3.20d), all the conditions for applying Lemma 4.1 are satisfied. Thus the first identity follows from the Lemma 4.1.

The second identity follows like the fifth; see below. The fourth identity follows from Corollary 4.2 with

$$
\begin{array}{ll}
\left(\mathbf{w}_{h}^{\prime}, \mathbf{u}_{h}^{\prime}, \mathbf{p}_{h}^{\prime}\right)=\left(\mathcal{W}_{\boldsymbol{\delta}_{t}}, \boldsymbol{U}_{\boldsymbol{\delta}_{t},}, \mathcal{P}_{\boldsymbol{\delta}_{t}}\right), & \left(\widehat{\mathbf{w}}_{h}^{\prime},\left(\widehat{\mathbf{u}}_{h}^{\prime}\right)_{t},\left(\widehat{\mathbf{u}}_{h}^{\prime}\right)_{n}, \widehat{\mathrm{p}}_{h}^{\prime}, \mathbf{f}^{\prime}\right)=\left(\boldsymbol{\delta}_{t},\left(\widehat{\boldsymbol{U}}_{\boldsymbol{\delta}_{t}}\right)_{t}, 0, \widehat{\mathcal{P}}_{\boldsymbol{\delta}_{t}}, \mathbf{0}\right), \\
\left(\mathbf{w}_{h}, \mathbf{u}_{h}, \mathrm{p}_{h}\right)=\left(\boldsymbol{\mathcal { W }}_{\boldsymbol{f}}, \boldsymbol{U}_{\boldsymbol{f}}, \mathcal{P}_{\boldsymbol{f}}\right), \quad\left(\widehat{\mathbf{w}}_{h},\left(\widehat{\mathbf{u}}_{h}\right)_{t},\left(\widehat{\mathbf{u}}_{h}\right)_{n}, \widehat{\mathrm{p}}_{h}, \mathbf{f}\right)=\left(\mathbf{0},\left(\widehat{\boldsymbol{u}}_{\boldsymbol{f}}\right)_{t}, \mathbf{0}, \widehat{\mathcal{P}}_{\boldsymbol{f}}, \boldsymbol{f}\right) .
\end{array}
$$

The fifth identity follows from Corollary 4.2 with

$$
\begin{aligned}
& \left(\mathbf{w}_{h}^{\prime}, \mathbf{u}_{h}^{\prime}, \mathbf{p}_{h}^{\prime}\right)=\left(\mathcal{W}_{\boldsymbol{\gamma}_{t}}, \mathcal{u}_{\gamma_{t}}, \mathcal{P}_{\boldsymbol{\gamma}_{t}}\right), \quad\left(\widehat{\mathbf{w}}_{h}^{\prime},\left(\widehat{\mathbf{u}}_{h}^{\prime}\right)_{t},\left(\widehat{\mathbf{u}}_{h}^{\prime}\right)_{n}, \widehat{\mathbf{p}}_{h}^{\prime}, \mathbf{f}^{\prime}\right)=\left(\gamma_{t},\left(\widehat{\boldsymbol{u}}_{\boldsymbol{\gamma}_{t}}\right)_{t}, 0, \widehat{\mathcal{P}}_{\boldsymbol{\gamma}_{t}}, \mathbf{0}\right), \\
& \left(\mathbf{w}_{h}, \mathbf{u}_{h}, \mathbf{p}_{h}\right)=\left(\boldsymbol{\mathcal { W }}_{\boldsymbol{\mu}_{n}}, \boldsymbol{u}_{\boldsymbol{\mu}_{n}}, \mathcal{P}_{\boldsymbol{\mu}_{n}}\right), \quad\left(\widehat{\mathbf{w}}_{h},\left(\widehat{\mathbf{u}}_{h}\right)_{t},\left(\widehat{\mathbf{u}}_{h}\right)_{n}, \widehat{\mathbf{p}}_{h}, \mathbf{f}\right)=\left(\mathbf{0},\left(\widehat{\boldsymbol{u}}_{\boldsymbol{\mu}_{n}}\right)_{t}, \boldsymbol{\mu}_{n}, \widehat{\mathcal{P}}_{\boldsymbol{\mu}_{n}}, \mathbf{0}\right) .
\end{aligned}
$$

The sixth identity follows from Lemma 4.1 with

$$
\begin{array}{ll}
\left(\mathbf{w}_{h}, \mathbf{u}_{h}, \mathrm{p}_{h}\right)=\left(\mathcal{W}_{\boldsymbol{\mu}_{n}}, \mathcal{u}_{\boldsymbol{\mu}_{n}}, \mathcal{P}_{\boldsymbol{\mu}_{n}}\right), & \left(\widehat{\mathbf{w}}_{h},\left(\widehat{\mathbf{u}}_{h}\right)_{t},\left(\widehat{\mathbf{u}}_{h}\right)_{n}, \widehat{\mathrm{p}}_{h}, \mathbf{f}\right)=\left(\mathbf{0},\left(\widehat{\mathbf{u}}_{\boldsymbol{\mu}_{n}}\right)_{t}, \boldsymbol{\mu}_{n}, \widehat{\mathcal{P}}_{\boldsymbol{\mu}_{n}}, \mathbf{0}\right), \\
\left(\mathbf{w}_{h}^{\prime}, \mathbf{u}_{h}^{\prime}, \mathbf{p}_{h}^{\prime}\right)=\left(\boldsymbol{\mathcal { W }}_{\boldsymbol{\lambda}_{n}}, \boldsymbol{u}_{\boldsymbol{\lambda}_{n}}, \mathcal{P}_{\boldsymbol{\lambda}_{n}}\right), & \left(\widehat{\mathbf{w}}_{h}^{\prime},\left(\widehat{\mathbf{u}}_{h}^{\prime}\right)_{t},\left(\widehat{\mathbf{u}}_{h}^{\prime}\right)_{n}, \widehat{\mathbf{p}}_{h}^{\prime}, \mathbf{f}^{\prime}\right)=\left(\mathbf{0},\left(\widehat{\boldsymbol{U}}_{\boldsymbol{\lambda}_{n}}\right)_{t}, \boldsymbol{\lambda}_{n}, \widehat{\mathcal{P}}_{\boldsymbol{\lambda}_{n}}, \mathbf{0}\right) .
\end{array}
$$

The eighth identity follows from Corollary 4.2 with

$$
\begin{array}{ll}
\left(\mathbf{w}_{h}, \mathbf{u}_{h}, \mathbf{p}_{h}\right)=\left(\mathcal{W}_{\boldsymbol{\mu}_{n}}, \boldsymbol{U}_{\boldsymbol{\mu}_{n},}, \mathcal{P}_{\boldsymbol{\mu}_{n}}\right), & \left(\widehat{\mathbf{w}}_{h},\left(\widehat{\mathbf{u}}_{h}\right)_{t},\left(\widehat{\mathbf{u}}_{h}\right)_{n}, \widehat{\mathbf{p}}_{h}, \mathbf{f}\right)=\left(\mathbf{0},\left(\widehat{\boldsymbol{u}}_{\boldsymbol{\mu}_{n}}\right)_{t}, \boldsymbol{\mu}_{n}, \widehat{\mathcal{P}}_{\boldsymbol{\mu}_{n}}, \mathbf{0}\right), \\
\left(\mathbf{w}_{h}^{\prime}, \mathbf{u}_{h}^{\prime}, \mathbf{p}_{h}^{\prime}\right)=\left(\mathcal{W}_{\boldsymbol{f}}, \boldsymbol{u}_{\boldsymbol{f}}, \mathcal{P}_{\boldsymbol{f}}\right), \quad\left(\widehat{\mathbf{w}}_{h}^{\prime},\left(\widehat{\mathbf{u}}_{h}^{\prime}\right)_{t},\left(\widehat{\mathbf{u}}_{h}^{\prime}\right)_{n}, \widehat{\mathbf{p}}_{h}^{\prime}, \mathbf{f}^{\prime}\right)=\left(\mathbf{0},\left(\widehat{\boldsymbol{u}}_{\boldsymbol{f}}\right)_{t}, \mathbf{0}, \widehat{\mathcal{P}}_{\boldsymbol{f}}, \boldsymbol{f}\right) .
\end{array}
$$

This completes the proof. $\square$

Proof of Theorem 3.6. By the jump conditions (3.22b) and (3.22c),

$$
\begin{aligned}
-\left\langle\llbracket\left(\widehat{\mathcal{U}}_{\gamma_{t}}+\widehat{\mathcal{U}}_{\boldsymbol{\lambda}_{n}^{o}}+\widehat{\mathcal{U}}_{\bar{\rho}}\right) \times \boldsymbol{n} \rrbracket, \boldsymbol{\delta}_{t}\right\rangle_{\mathscr{E}_{h}} & =\left\langle\llbracket\left(\widehat{\mathcal{U}}_{\boldsymbol{f}_{t}}+\widehat{\mathcal{U}}_{\boldsymbol{g}_{n}}\right) \times \boldsymbol{n} \rrbracket, \boldsymbol{\delta}_{t}\right\rangle_{\mathscr{E}_{h}}-\left\langle\boldsymbol{g}_{t} \times \boldsymbol{n}, \boldsymbol{\delta}_{t}\right\rangle_{\partial \Omega}, \\
-\left\langle\llbracket\left(\widehat{\mathcal{P}}_{\boldsymbol{\gamma}_{t}}+\widehat{\mathcal{P}}_{\boldsymbol{\lambda}_{n}^{o}}+\widehat{\mathcal{P}}_{\bar{\rho}}\right) \boldsymbol{n} \rrbracket, \boldsymbol{\mu}_{n}\right\rangle_{\mathscr{E}_{h}} & =\left\langle\llbracket\left(\widehat{\mathcal{P}}_{\boldsymbol{f}_{t}}+\widehat{\mathcal{P}}_{\boldsymbol{g}_{n}}\right) \boldsymbol{n} \rrbracket, \boldsymbol{\mu}_{n}\right\rangle_{\mathscr{E}_{h}} .
\end{aligned}
$$

By Lemma 4.5, we have that

$$
\begin{array}{rlrl}
-\left\langle\llbracket \widehat{\mathcal{U}}_{\gamma_{t}} \times \boldsymbol{n} \rrbracket, \boldsymbol{\delta}_{t}\right\rangle_{\mathscr{E}_{h}} & =a_{h}\left(\boldsymbol{\lambda}_{t}^{o}, \boldsymbol{\delta}_{t}\right), & -\left\langle\llbracket \widehat{\mathcal{P}}_{\gamma_{t}} \boldsymbol{n} \rrbracket, \boldsymbol{\mu}_{n}\right\rangle_{\mathscr{E}_{h}} & =-b_{h}\left(\boldsymbol{\mu}_{n}, \boldsymbol{\gamma}_{t}\right), \\
-\left\langle\llbracket \widehat{\mathcal{U}}_{\boldsymbol{\lambda}_{n}^{o}} \times \boldsymbol{n} \rrbracket, \boldsymbol{\delta}_{t}\right\rangle_{\mathscr{E}_{h}} & =b_{h}\left(\boldsymbol{\lambda}_{n}^{o}, \boldsymbol{\delta}_{t}\right), & -\left\langle\llbracket \widehat{\mathcal{P}}_{\boldsymbol{\lambda}_{n}^{o}} \boldsymbol{n} \rrbracket, \boldsymbol{\mu}_{n}\right\rangle_{\mathscr{E}_{h}} & =c_{h}\left(\boldsymbol{\lambda}_{n}^{o}, \boldsymbol{\mu}_{n}\right), \\
-\left\langle\llbracket \widehat{\mathcal{U}}_{\bar{\rho}} \times \boldsymbol{n} \rrbracket, \boldsymbol{\delta}_{t}\right\rangle_{\mathscr{E}_{h}}=0, & & -\left\langle\llbracket \widehat{\mathcal{P}}_{\bar{\rho}} \boldsymbol{n} \rrbracket, \boldsymbol{\mu}_{n}\right\rangle_{\mathscr{E}_{h}}=d\left(\bar{\rho}, \boldsymbol{\mu}_{n}\right) .
\end{array}
$$

It remains to show that $\ell_{1}=\tilde{\ell}_{1}$ and $\ell_{2}=\tilde{\ell}_{2}$ where

$$
\begin{aligned}
\tilde{\ell}_{1}\left(\boldsymbol{\delta}_{t}\right) & :=-\left\langle\llbracket\left(\widehat{\boldsymbol{u}}_{\boldsymbol{f}}+\widehat{\boldsymbol{U}}_{\boldsymbol{g}_{n}}\right) \times \boldsymbol{n} \rrbracket, \boldsymbol{\delta}_{t}\right\rangle_{\mathscr{E}_{h}}-\left\langle\boldsymbol{g}_{t} \times \boldsymbol{n}, \boldsymbol{\delta}_{t}\right\rangle_{\partial \Omega}, \\
\tilde{\ell}_{2}(\psi) & :=\left\langle\llbracket\left(\widehat{\mathcal{P}}_{\boldsymbol{f}_{t}}+\widehat{\mathcal{P}}_{\boldsymbol{g}_{n}}\right) \boldsymbol{n} \rrbracket, \boldsymbol{\mu}_{n}\right\rangle_{\mathscr{E}_{h}} .
\end{aligned}
$$


But, again by Lemma 4.5, we have

$$
\begin{aligned}
\tilde{\ell}_{1}\left(\boldsymbol{\delta}_{t}\right) & =-\left(\boldsymbol{f}, \boldsymbol{U}_{\boldsymbol{\delta}_{t}}\right)_{\Omega_{h}}-b_{h}\left(\boldsymbol{g}_{n}, \boldsymbol{\delta}_{t}\right)-\left\langle\boldsymbol{g}_{t} \times \boldsymbol{n}, \boldsymbol{\delta}_{t}\right\rangle_{\partial \Omega} \\
& =\ell_{1}\left(\boldsymbol{\delta}_{t}\right),
\end{aligned}
$$

and, similarly, by Lemma 4.5 ,

$$
\begin{aligned}
\tilde{\ell}_{2}\left(\boldsymbol{\mu}_{n}\right) & =\left(\boldsymbol{f}, \boldsymbol{u}_{\boldsymbol{\mu}_{n}}\right)_{\Omega_{h}}-c_{h}\left(\boldsymbol{g}_{n}, \boldsymbol{\mu}_{n}\right) \\
& =\ell_{2}\left(\boldsymbol{\mu}_{n}\right) .
\end{aligned}
$$

The proof of Theorem 3.6 is completed by also establishing the uniqueness as in the previous cases.

4.5. Proof of the characterization of Theorem 3.8. To prove Theorem 3.8, we use the identities below. The numerical traces of the form $\widehat{\boldsymbol{u}}_{\odot}$ appearing in these identities are defined using (3.24e) and (3.24f) as in the previous cases for all possible choices of the subscripts $\odot$ that make sense for this case.

Lemma 4.6 (Elementary identities). For any $\boldsymbol{\gamma}_{t}, \boldsymbol{\delta}_{t} \in \boldsymbol{L}^{2}\left(\mathscr{E}_{h}\right)$, any $\rho, \psi \in L^{2}\left(\mathscr{E}_{h}\right)$, any $\phi \in H^{1}\left(\Omega_{h}\right)$ and any $\boldsymbol{f} \in \boldsymbol{L}^{2}(\Omega)$, we have

$$
\begin{aligned}
& -\left\langle\llbracket \widehat{\mathcal{U}}_{\gamma_{t}} \times \boldsymbol{n} \rrbracket, \boldsymbol{\delta}_{t}\right\rangle_{\mathscr{E}_{h}}=\left(\mathcal{W}_{\gamma_{t}}, \mathcal{W}_{\boldsymbol{\delta}_{t}}\right)_{\Omega_{h}}+\left\langle\frac{1}{\tau_{n}} \mathcal{P}_{\gamma_{t}}, \mathcal{P}_{\boldsymbol{\delta}_{t}}\right\rangle_{\partial \Omega_{h}} \\
& +\left\langle\frac{1}{\tau_{t}} \boldsymbol{n} \times\left(\gamma_{t}-\mathcal{W}_{\gamma_{t}}\right), \boldsymbol{n} \times\left(\boldsymbol{\delta}_{t}-\mathcal{W}_{\boldsymbol{\delta}_{t}}\right)\right\rangle_{\partial \Omega_{h}}, \\
& -\left\langle\llbracket \widehat{\boldsymbol{U}}_{\rho} \times \boldsymbol{n} \rrbracket, \boldsymbol{\delta}_{t}\right\rangle_{\mathscr{E}_{h}}=-\left\langle\llbracket \widehat{\boldsymbol{U}}_{\boldsymbol{\delta}_{t}} \cdot \boldsymbol{n} \rrbracket, \rho\right\rangle_{\mathscr{E}_{h}}, \\
& -\left\langle\llbracket \widehat{\boldsymbol{u}}_{\phi} \times \boldsymbol{n} \rrbracket, \boldsymbol{\delta}_{t}\right\rangle_{\mathscr{E}_{h}}=\left\langle\boldsymbol{n} \times \operatorname{grad} \phi, \boldsymbol{\delta}_{t}\right\rangle_{\partial \Omega_{h}}, \\
& -\left\langle\llbracket \widehat{\mathcal{U}}_{\boldsymbol{f}} \times \boldsymbol{n} \rrbracket, \boldsymbol{\delta}_{t}\right\rangle_{\mathscr{E}_{h}}=+\left(\boldsymbol{f}, \mathcal{u}_{\boldsymbol{\delta}_{t}}\right)_{\Omega_{h}},
\end{aligned}
$$

and

$$
\begin{aligned}
& -\left\langle\llbracket \widehat{\mathcal{U}}_{\gamma_{t}} \cdot \boldsymbol{n} \rrbracket, \psi\right\rangle_{\mathscr{E}_{h}}=-\left\langle\llbracket \widehat{\boldsymbol{U}}_{\psi} \times \boldsymbol{n} \rrbracket, \gamma_{t}\right\rangle_{\mathscr{E}_{h}} \\
& -\left\langle\llbracket \widehat{\mathfrak{U}}_{\rho} \cdot \boldsymbol{n} \rrbracket, \psi\right\rangle_{\mathscr{E}_{h}}=\left(\mathcal{W}_{\rho}, \mathcal{W}_{\psi}\right)_{\Omega_{h}}+\left\langle\frac{1}{\tau_{n}}\left(\rho-\mathcal{P}_{\rho}\right),\left(\psi-\mathcal{P}_{\psi}\right)\right\rangle_{\partial \Omega_{h}} \\
& +\left\langle\frac{1}{\tau_{t}} \boldsymbol{n} \times \mathcal{W}_{\rho}, \boldsymbol{n} \times \mathcal{W}_{\psi}\right\rangle_{\partial \Omega_{h}}, \\
& -\left\langle\llbracket \widehat{\boldsymbol{u}}_{\phi} \cdot \boldsymbol{n} \rrbracket, \psi\right\rangle_{\mathscr{E}_{h}}=-\langle\operatorname{grad} \phi \cdot \boldsymbol{n}, \psi\rangle_{\partial \Omega_{h}}, \\
& -\left\langle\llbracket \widehat{\mathcal{U}}_{\boldsymbol{f}} \cdot \boldsymbol{n} \rrbracket, \psi\right\rangle_{\mathscr{E}_{h}}=+\left(\boldsymbol{f}, \boldsymbol{u}_{\psi}\right)_{\Omega_{h}} .
\end{aligned}
$$

Proof. The third and seventh identities are immediate because (3.28) implies that

$$
\widehat{\mathfrak{u}}_{\phi}=\operatorname{grad} \phi .
$$

In the remainder of the proof, whenever we apply Lemma 4.1 or Corollary 4.2 we take $\mathbf{P} \boldsymbol{v}=\operatorname{grad} \phi_{\boldsymbol{v}}$ and $\mathrm{P}=0$. To prove the first identity, we proceed as in the previous cases and apply Lemma 4.1 (now additionally noting that $\mathbf{P} \boldsymbol{U}_{\gamma_{t}}=\mathbf{0}$ ) with

$$
\begin{aligned}
& \left(\mathbf{w}_{h}, \mathbf{u}_{h}, \mathrm{p}_{h}\right)=\left(\boldsymbol{W}_{\boldsymbol{\gamma}_{t}}, \boldsymbol{U}_{\boldsymbol{\gamma}_{t}}, \mathcal{P}_{\boldsymbol{\gamma}_{t}}\right), \\
& \left(\widehat{\mathbf{w}}_{h}, \widehat{\mathbf{u}}_{h}, \widehat{\mathrm{p}}_{h}, \mathbf{f}\right)=\left(\gamma_{t}, \widehat{\boldsymbol{u}}_{\gamma_{t}}, 0, \mathbf{0}\right), \\
& \left(\mathbf{w}_{h}^{\prime}, \mathbf{u}_{h}^{\prime}, \mathbf{p}_{h}^{\prime}\right)=\left(\mathcal{W}_{\boldsymbol{\delta}_{t}}, \boldsymbol{U}_{\boldsymbol{\delta}_{t}}, \mathcal{P}_{\boldsymbol{\delta}_{t}}\right), \\
& \left(\widehat{\mathbf{w}}_{h}^{\prime}, \widehat{\mathbf{u}}_{h}^{\prime}, \widehat{\mathbf{p}}_{h}^{\prime}, \mathbf{f}^{\prime}\right)=\left(\boldsymbol{\delta}_{t}, \widehat{\boldsymbol{u}}_{\boldsymbol{\delta}_{t}}, 0, \mathbf{0}\right) \text {. }
\end{aligned}
$$


The second identity is proved just like the fifth; see below. The fourth identity follows from Corollary 4.2 with

$$
\begin{array}{ll}
\left(\mathbf{w}_{h}, \mathbf{u}_{h}, \mathrm{p}_{h}\right)=\left(\mathcal{W}_{\boldsymbol{f}}, \boldsymbol{u}_{\boldsymbol{f}}, \mathcal{P}_{\boldsymbol{f}}\right), & \left(\widehat{\mathbf{w}}_{h}, \widehat{\mathbf{u}}_{h}, \widehat{\mathrm{p}}_{h}, \mathbf{f}\right)=\left(0, \widehat{\boldsymbol{u}}_{\boldsymbol{f}}, 0, \boldsymbol{f}\right), \\
\left(\mathbf{w}_{h}^{\prime}, \mathbf{u}_{h}^{\prime}, \mathrm{p}_{h}^{\prime}\right)=\left(\mathcal{W}_{\boldsymbol{\delta}_{t}}, \boldsymbol{u}_{\boldsymbol{\delta}_{t}}, \mathcal{P}_{\boldsymbol{\delta}_{t}}\right), & \left(\widehat{\mathbf{w}}_{h}^{\prime}, \widehat{\mathbf{u}}_{h}^{\prime}, \widehat{\mathrm{p}}_{h}^{\prime}, \mathbf{f}^{\prime}\right)=\left(\boldsymbol{\delta}_{t}, \widehat{\boldsymbol{u}}_{\boldsymbol{\delta}_{t}}, 0, \mathbf{0}\right),
\end{array}
$$

The fifth identity follows from Corollary 4.2 with

$$
\begin{array}{ll}
\left(\mathbf{w}_{h}, \mathbf{u}_{h}, \mathrm{p}_{h}\right)=\left(\mathcal{W}_{\gamma_{t}}, \mathcal{u}_{\gamma_{t}}, \mathcal{P}_{\gamma_{t}}\right), & \left(\widehat{\mathbf{w}}_{h}, \widehat{\mathbf{u}}_{h}, \widehat{\mathrm{p}}_{h}, \mathbf{f}\right)=\left(\gamma_{t}, \widehat{\mathbf{u}}_{\left.\gamma_{t}, 0,0\right),}\right) \\
\left(\mathbf{w}_{h}^{\prime}, \mathbf{u}_{h}^{\prime}, \mathrm{p}_{h}^{\prime}\right)=\left(\mathcal{W}_{\psi}, \boldsymbol{u}_{\psi}, \mathcal{P}_{\psi}\right), & \left(\widehat{\mathbf{w}}_{h}^{\prime}, \widehat{\mathbf{u}}_{h}^{\prime}, \widehat{\mathrm{p}}_{h}^{\prime}, \mathbf{f}^{\prime}\right)=\left(0, \widehat{\mathfrak{u}}_{\psi}, \psi, \mathbf{0}\right) .
\end{array}
$$

The sixth identity follows from Lemma 4.1 with

$$
\begin{array}{ll}
\left(\mathbf{w}_{h}, \mathbf{u}_{h}, \mathrm{p}_{h}\right)=\left(\mathcal{W}_{\rho}, \boldsymbol{u}_{\rho}, \mathcal{P}_{\rho}\right), & \left(\widehat{\mathbf{w}}_{h}, \widehat{\mathbf{u}}_{h}, \widehat{\mathrm{p}}_{h}, \mathbf{f}\right)=\left(0, \widehat{\boldsymbol{u}}_{\rho}, \rho, \mathbf{0}\right) . \\
\left(\mathbf{w}_{h}^{\prime}, \mathbf{u}_{h}^{\prime}, \mathbf{p}_{h}^{\prime}\right)=\left(\mathcal{W}_{\psi}, \boldsymbol{u}_{\psi}, \mathcal{P}_{\psi}\right), & \left(\widehat{\mathbf{w}}_{h}^{\prime}, \widehat{\mathbf{u}}_{h}^{\prime}, \widehat{\mathbf{p}}_{h}^{\prime}, \mathbf{f}^{\prime}\right)=\left(0, \widehat{\boldsymbol{u}}_{\psi}, \psi, \mathbf{0}\right) .
\end{array}
$$

The eighth identity follows from Corollary 4.2 with

$$
\begin{array}{ll}
\left(\mathbf{w}_{h}, \mathbf{u}_{h}, \mathrm{p}_{h}\right)=\left(\mathcal{W}_{\boldsymbol{f}}, \boldsymbol{u}_{\boldsymbol{f}}, \mathcal{P}_{\boldsymbol{f}}\right), & \left(\widehat{\mathbf{w}}_{h}, \widehat{\mathbf{u}}_{h}, \widehat{\mathrm{p}}_{h}, \mathbf{f}\right)=\left(0, \widehat{\mathbf{u}}_{\boldsymbol{f}}, 0, \boldsymbol{f}\right), \\
\left(\mathbf{w}_{h}^{\prime}, \mathbf{u}_{h}^{\prime}, \mathbf{p}_{h}^{\prime}\right)=\left(\mathcal{W}_{\psi}, \boldsymbol{u}_{\psi}, \mathcal{P}_{\psi}\right), & \left(\widehat{\mathbf{w}}_{h}^{\prime}, \widehat{\mathbf{u}}_{h}^{\prime}, \widehat{\mathrm{p}}_{h}^{\prime}, \mathbf{f}^{\prime}\right)=\left(0, \widehat{\boldsymbol{u}}_{\psi}, \psi, \mathbf{0}\right) .
\end{array}
$$

Proof of Theorem 3.8. By the jump conditions (3.27b) and (3.27c),

$$
\begin{aligned}
-\left\langle\llbracket\left(\widehat{\boldsymbol{u}}_{\gamma_{t}}+\widehat{\boldsymbol{U}}_{\rho}+\widehat{\boldsymbol{U}}_{\phi}\right) \times \boldsymbol{n} \rrbracket, \boldsymbol{\delta}_{t}\right\rangle_{\mathscr{E}_{h}} & =\left\langle\llbracket\left(\widehat{\boldsymbol{U}}_{\boldsymbol{f}} \times \boldsymbol{n} \rrbracket, \boldsymbol{\delta}_{t}\right\rangle_{\mathscr{E}_{h}}-\left\langle\boldsymbol{g} \times \boldsymbol{n}, \boldsymbol{\delta}_{t}\right\rangle_{\partial \Omega},\right. \\
-\left\langle\llbracket\left(\widehat{\boldsymbol{u}}_{\gamma_{t}}+\widehat{\boldsymbol{u}}_{\rho}+\widehat{\mathfrak{u}}_{\phi}\right) \cdot \boldsymbol{n} \rrbracket, \psi\right\rangle_{\mathscr{E}_{h}} & =\left\langle\llbracket \widehat{\mathfrak{u}}_{\boldsymbol{f}} \cdot \boldsymbol{n} \rrbracket, \psi\right\rangle_{\mathscr{E}_{h}}-\langle\boldsymbol{g} \cdot \boldsymbol{n}, \psi\rangle_{\partial \Omega},
\end{aligned}
$$

By Lemma 4.6, we have that

$$
\begin{aligned}
& -\left\langle\llbracket \widehat{\mathcal{U}}_{\boldsymbol{\gamma}_{t}} \times \boldsymbol{n} \rrbracket, \boldsymbol{\delta}_{t}\right\rangle_{\mathscr{E}_{h}}=a_{h}\left(\boldsymbol{\gamma}_{t}, \boldsymbol{\delta}_{t}\right), \quad-\left\langle\llbracket \widehat{\boldsymbol{U}}_{\boldsymbol{\gamma}_{t}} \cdot \boldsymbol{n} \rrbracket, \psi\right\rangle_{\mathscr{E}_{h}}=b_{h}\left(\psi, \boldsymbol{\gamma}_{t}\right), \\
& -\left\langle\llbracket \widehat{\mathcal{U}}_{\rho} \times \boldsymbol{n} \rrbracket, \boldsymbol{\delta}_{t}\right\rangle_{\mathscr{E}_{h}}=b_{h}\left(\rho, \boldsymbol{\delta}_{t}\right), \quad-\left\langle\llbracket \widehat{\boldsymbol{u}}_{\rho} \cdot \boldsymbol{n} \rrbracket, \psi\right\rangle_{\mathscr{E}_{h}}=d_{h}(\rho, \psi), \\
& -\left\langle\llbracket \widehat{\boldsymbol{u}}_{\phi} \times \boldsymbol{u} \rrbracket, \boldsymbol{\delta}_{t}\right\rangle_{\mathscr{E}_{h}}=c_{h}\left(\phi, \boldsymbol{\delta}_{t}\right), \quad-\left\langle\llbracket \widehat{\boldsymbol{u}}_{\phi} \cdot \boldsymbol{n} \rrbracket, \psi\right\rangle_{\mathscr{E}_{h}}=e_{h}(\phi, \psi),
\end{aligned}
$$

and that

$$
\begin{aligned}
\left\langle\llbracket\left(\widehat{\boldsymbol{u}}_{\boldsymbol{f}} \times \boldsymbol{n} \rrbracket, \boldsymbol{\delta}_{t}\right\rangle_{\mathscr{E}_{h}}-\left\langle\boldsymbol{g} \times \boldsymbol{n}, \boldsymbol{\delta}_{t}\right\rangle_{\partial \Omega}\right. & =\ell_{1}\left(\boldsymbol{\delta}_{t}\right), \\
\left\langle\llbracket \widehat{\boldsymbol{u}}_{\boldsymbol{f}} \cdot \boldsymbol{n} \rrbracket, \psi\right\rangle_{\mathscr{E}_{h}}-\langle\boldsymbol{g} \cdot \boldsymbol{n}, \psi\rangle_{\partial \Omega} & =\ell_{2}(\psi) .
\end{aligned}
$$

The proof of Theorem 3.8 is now completed by a uniqueness argument as in the previous cases.

5. Concluding remarks. In this paper, we introduced a new HDG method for the Stokes system and showed four different ways of hybridizing it. In order for these methods to be competitive with previously known ones [14, 20, 18, 19, 12, 15, 3, 13], they need to be, not only efficiently implemented, but also efficiently solved. We would like to emphasize that our characterization theorems are a first step towards such a goal since they shed light on the structure of the corresponding equations. However, we feel that a meaningful study of those equations deserves a separate paper. The design of efficient solvers for these methods constitutes work in progress. 
Another subject that constitutes the subject of ongoing work is the analysis of the accuracy of the methods. A careful a priori error analysis of the HDG methods should reveal what is the effect of the choice of the stabilization parameters $\tau_{n}$ and $\tau_{t}$ on their accuracy. Let us recall that, in the context of second-order elliptic problems, the HDG methods [10] were shown to be more accurate than all previously known DG methods when their stabilization parameters are suitably chosen. In particular, when using polynomial approximations of the same degree for both the solution and its gradient, both approximations were shown to converge with optimal order; see [4, 11]. It is thus reasonable to expect that by a proper choice of the parameters $\tau_{n}$ and $\tau_{t}$, the HDG method using polynomial approximations of the same degree for the vorticity, velocity and pressure, will also converge optimally in all three variables. This is work in progress.

\section{REFERENCES}

[1] D. N. Arnold, F. Brezzi, B. Cockburn, and L. D. Marini, Unified analysis of discontinuous Galerkin methods for elliptic problems, SIAM J. Numer. Anal. 39 (2002), 1749-1779.

[2] F. Brezzi and M. Fortin, Mixed and hybrid finite element methods, Springer Verlag, 1991.

[3] J. Carrero, B. Cockburn, and D. Schötzau, Hybridized, globally divergence-free LDG methods. Part I: The Stokes problem, Math. Comp. 75 (2006), 533-563.

[4] B. Cockburn, B. Dong, and J. Guzmán, A superconvergent LDG-hybridizable Galerkin method for second-order elliptic problems, Math. Comp., to appear.

[5] B. Cockburn and J. Gopalakrishnan, A characterization of hybridized mixed methods for second order elliptic problems, SIAM J. Numer. Anal. 42 (2004), 283-301.

[6] - Error analysis of variable degree mixed methods for elliptic problems via hybridization, Math. Comp. 74 (2005), 1653-1677.

[7] _ Incompressible finite elements via hybridization. Part I: The Stokes system in two space dimensions, SIAM J. Numer. Anal. 43 (2005), 1627-1650.

[8] - Incompressible finite elements via hybridization. Part II: The Stokes system in three space dimensions, SIAM J. Numer. Anal. 43 (2005), 1651-1672.

[9] — New hybridization techniques, GAMM-Mitt. 2 (2005), 154-183.

[10] B. Cockburn, J. Gopalakrishnan, and R. Lazarov, Unified hybridization of discontinuous Galerkin, mixed and continuous Galerkin methods for second order elliptic problems, SIAM J. Numer. Anal., Submitted.

[11] B. Cockburn, J. Guzmán, and H. Wang, Superconvergent discontinuous Galerkin methods for second-order elliptic problems, Math. Comp., to appear.

[12] B. Cockburn, G. Kanschat, and D. Schötzau, A locally conservative LDG method for the incompressible Navier-Stokes equations, Math. Comp. 74 (2005), 1067-1095.

[13] A note on discontinuous Galerkin divergence-free solutions of the Navier-Stokes equations, J. Sci. Comput. 31 (2007), 61-73.

[14] B. Cockburn, G. Kanschat, D. Schötzau, and C. Schwab, Local discontinuous Galerkin methods for the Stokes system, SIAM J. Numer. Anal. 40 (2002), 319-343.

[15] V. Girault, B. Rivière, and M. F. Wheeler, A discontinuous Galerkin method with nonoverlapping domain decomposition for the Stokes and Navier-Stokes problems, Math. Comp. 74 (2005), 53-84.

[16] M. D. Gunzburger, Finite element methods for viscous incompressible flows: A guide to theory, practice and algorithms, Academic Press, 1989.

[17] J.-C. Nédélec, Éléments finis mixtes incompressibles pour l'équation de Stokes dans $\mathbf{R}^{3}$, Numer. Math. 39 (1982), 97-112.

[18] D. Schötzau, C. Schwab, and A. Toselli, hp-DGFEM for incompressible flows, SIAM J. Numer. Anal. 40 (2003), 2171-2194.

[19] D. Schötzau, C. Schwab, and A. Toselli, Stabilized hp-DGFEM for incompressible flow, Math. Models Methods Appl. Sci. 13 (2003), 1413-1436.

[20] A. Toselli, hp-discontinuous Galerkin approximations for the Stokes problem, Math. Models Methods Appl. Sci. 12 (2002), 1565-1616.

[21] J. Wang and X. Ye, New finite element methods in computational fluid dynamics by H(div) elements, SIAM J. Numer. Anal. 45 (2007), 1269-1286. 